\title{
Alterations in membrane structure and metabolism in ischemic and reperfused myocardium
}

Citation for published version (APA):

Schrijvers, A. H. G. J. (1989). Alterations in membrane structure and metabolism in ischemic and reperfused myocardium. [Doctoral Thesis, Maastricht University]. Rijksuniversiteit Limburg. https://doi.org/10.26481/dis.19891124as

Document status and date:

Published: 01/01/1989

DOI:

10.26481/dis.19891124as

Document Version:

Publisher's PDF, also known as Version of record

\section{Please check the document version of this publication:}

- A submitted manuscript is the version of the article upon submission and before peer-review. There can be important differences between the submitted version and the official published version of record.

People interested in the research are advised to contact the author for the final version of the publication, or visit the DOI to the publisher's website.

- The final author version and the galley proof are versions of the publication after peer review.

- The final published version features the final layout of the paper including the volume, issue and page numbers.

Link to publication

\footnotetext{
General rights rights.

- You may freely distribute the URL identifying the publication in the public portal. please follow below link for the End User Agreement:

www.umlib.nl/taverne-license

Take down policy

If you believe that this document breaches copyright please contact us at:

repository@maastrichtuniversity.nl

providing details and we will investigate your claim.
}

Copyright and moral rights for the publications made accessible in the public portal are retained by the authors and/or other copyright owners and it is a condition of accessing publications that users recognise and abide by the legal requirements associated with these

- Users may download and print one copy of any publication from the public portal for the purpose of private study or research.

- You may not further distribute the material or use it for any profit-making activity or commercial gain

If the publication is distributed under the terms of Article $25 \mathrm{fa}$ of the Dutch Copyright Act, indicated by the "Taverne" license above, 


\section{ALTERATIONS IN MEMBRANE STRUCTURE AND METABOLISM IN ISCHEMIC AND REPERFUSED MYOCARDIUM}

\section{PROEFSCHRIFT}

ter verkrijging van de graad van doctor an de Rijksuniversiteil Limburg te Maastricht, op gezag van de Rector Magnificus. Prof. Dr. F.I.M. Bonke, volgens het besluit van het College van Dekanen, in thet openbaat te verdedigen op vrijdag. 24 november 1989. om 14.00 uur

door

Andreas Henricus Gerardus Joannes Schrijvers

geboren te Aalsi 
Promotores: $\quad$ Prof. Dr. R.S. Reneman.

Prof. Dr. G.J. van der Vusse.

Co-promotor: $\quad$ Dr. P.M. Frederik.

Leden wan de beoordelingscommissie:

Prof, Dr. R.F.A. Zwaal (voorzitter),

Prof. Dr, F.T. Basman.

Dr. W. Th. Hermens.

Prof:. Dr. W.C. Hülsmann,

Prof. Dr. A.J. Verkleij.

The study presented in this thesis was financially supported by Medigon-NWO (Grant $900-516-91)$.

Financial support by the Netherland Heart Foundation, the Interuniversity Cardiologic Instütution Netherland and the Wijnand M. Pon foundation (Leusden) for the publication of this thesis are gratefully acknowledged.

Druk: Krips Repro, Meppel. 
Voor mijn ouders

Aan Anila on Sander 


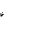




\section{CONTENTS}

\section{CHAPTER \# GENERAL INTRODUCTION}

1.1. Introduction.

1.2. Alterations in cardiac membranes during ischemia and reperfusion.

1.3. Multilamellar structures.

1.4. Alterations in myocardial (lipid) metabolism.

1.5. Fixation of lissue lipids.

1.6. Aim of the study.

1.7. Experimental approach.

CHAPTER 2 DUAL EFFECT OF TANNIC ACID ON THE PRESERVATION AND ULTRASTRUCTURE OF PHOSPHATIDYLCHOLINE VESICLES

2. I. Introduction.

2.2. Experimental procedures.

2.3. Results.

2.4. Discussion.

2.5. Summary.

CHAPTER 3 THE FORMATION OF MULT'ILAMELLAR VESICLES BY THE ADDITION OF "TANNIC ACID TO PHOSPHATIDYLCHOLINE CONTAINING SMALL UNILAMELLAR VESICILES

3. Introduction.

3.2. Material and methodls.

3.3. Results.

3.4. Discussion. 
CHAPTER INFLUENCE OF THE DURATION OF ISCHEMLA AND REPERFUSION ON MULTILAMELLAR VESICLES IN ISOLATED RABBIT HEARTS: AN ULTRASTRUCTURAL AND MORPHOMETRICAL STUDY USIING TANNIC ACID BASED FIXATION

4. II. Introduction.

4.2. Material and methods.

4.3. Results.

4.4. Discussion.

4.5. Summary.

CHAPTER 5 CORRELATION IN TIME BETWEEN THE INCREASE OF MULTILAMELLAR VESICLES AND METABOLIC ALTERATIONS IN ISCHEMIC AND REPERFUSED RABBIT HEARTS

5.1. Introduction.

5.2. Matterial and methods.

5.3. Resulis.

5.4. Discussion.

5.5. Summary.

CHAPTER 6 MULTILAMELLAR EXTRUSIONS AND ALTERATIONS IN LIPID METABOLISM IN ISOLATED, ISCHEMIC AND REPERFUSED RAT HEARTS: A COMPARISON WITH RABBIT HEARTS

6.1. Introduction.

6.2. Material and methods.

6.3. Results.

6.4. Discussion.

6.5. Summary. 


\section{CHAPTER 7 GENERAL DISCUSSION}

7.1. Membrane alterations and cell damage.

7.2. Tannic acid interaction(s) with phospholipids in a bilayered configuration.

7.3. The use of tannic acid based fixation in ischemic and reparfused myocardium.

7.4. Species differences or similarities in the formation of multilamellar vesicles.

7.5. Enzyme release (LDH) an indicator of membrane injury.

7.6. ATP depletion, lipid changes and cell damage.

7.7. Glycocalyx alterations and cell damage.

7.8. Altered cytoskeleton interactions and cell damage.

7.9. Membrane protein disorders and cell damage. 

CHAPTER I

GENERAL INTRODUCTION 


\section{I.I INTRODUCTION}

There is increasing evidence that the development of myocardial membrane dysfunction is of considerable importance in the pathogenesis of ischemic myocardial cell injury. A large number of experimental findings indicates that alterations in myocardial lipid metabolism can alter cardiac function by changing the properties of cardiac cell membranes, ultimately leading to cell cleath. While the precise mechanism of membrane dysfunction is not entirely clear, several studies have focussed on the possible role of alterations in membrane phospholipid content and composition. In addition, the generation of amphipathic metabolites, which may directly affect sarcolemmal integrity, has also been documented. Beside metabolic studies, considerable effort has been made to study morphological alterations representative of ischemia induced membrane damage. A variety of structural changes has been reported so far, but quantitative studies and comparisons of ultrastructural findings and metabolic parameters are scarce. Little is known about the relation between additional alterations in lipid metabolism and increased cell damage during reperfusion.

\subsection{ALTERATIONS IN CARDIAC MEMBRANES DURING ISCHEMIA AND REPER- FUSION}

\subsubsection{Membrane architecture in general.}

All biological membranes, including the plasma membrane and intracellular membranes of myocardial cells. have a common overall structure: they are a composition of lipid and protein molecules with non-covalent interactions forming the plasma membrane (Fig. 1.1). The lipid molecules are arranged in a continuous double layer of 4 to $5 \mathrm{~mm}$. The lipid bilayer provides the basic structure of the membrane and serves as a relative impermeable barrier for most water-soluble molecules. The membrane proteins in the lipid bilayer are involved in the various specific functions of the membranes, such as receptors for hormones or other signal molecules, membrane-bound enzymes, and mediators of active transport of ions and macromolecules through the 


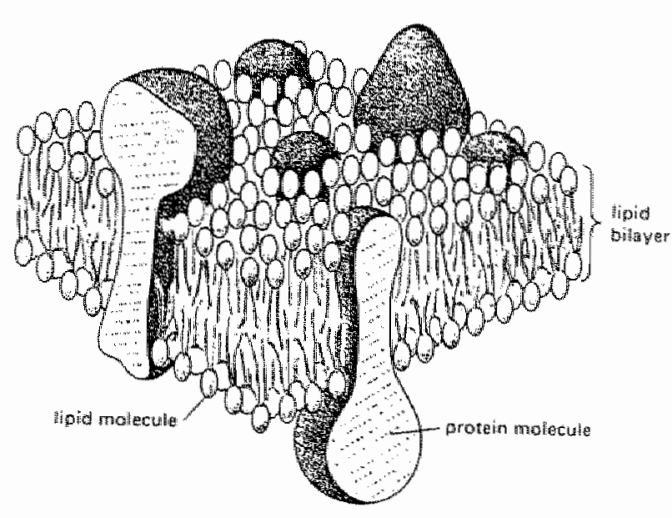

\begin{abstract}
Figure M.I Sichemanic thredimensionat view of a small section of a plasma menbrowe, about $10 \mathrm{~mm}$ square. Membrane proteins floaing iir a sea of phospholipids faffer: Alberis et al., 1983).
\end{abstract}

membrane. The membrane lipids and proteins can move freely in the plane of the membrane. These dynamic aspects of the membrane structure have been described by Singer and Nicholson (206) in the "fluid mosaic model" (Fig. 1.1). It has been recognized, however, that the membrane structure is more complex; membrane lipids may exist in discrete domains, which may differ greatly in their composition and properties, thereby modulating membrane protein function $(39,182,262,265)$.

Unlike bacterial plasma membranes, which are often composed of one major type of phospholipid and contain no cholesterol, the plasma membrane and intracellular membranes of most eukaryotic cells contain not only large quantities of cholesterol, but also a larger variety of phospholipids.

Phospholipids include sphingomyelin and several phosphoglycerides (Fig. 1.2), containing either one of five different polar head groups: hydroxyl (phosphatidic acid), serine (phosphatidylserine), inositol (phosphatidylinositol), êthanolamine (phosphatidylethanolamine), and choline (phosphatidylcholine). At physiological $\mathrm{H}^{+}$ concentration $(\mathrm{pH} 7.4)$ the choline-phosphate group is a zwitterion with a single unit positive charge at the amino group and a single negative charge at the phosphate group; its net charge is neutral, but with a quite polar group. The ethanolamine head

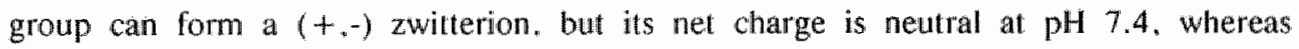
phosphatidylserine forms a $(+,-$.$) net negative ion. The polar head group of phospha-$ tidylinositol is a hexahydroxy alcohol rather than an amine, it is net negative at $\mathrm{pH}$ 7.4 . 


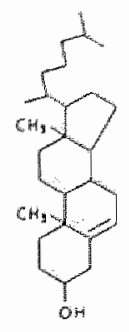

Cholutale

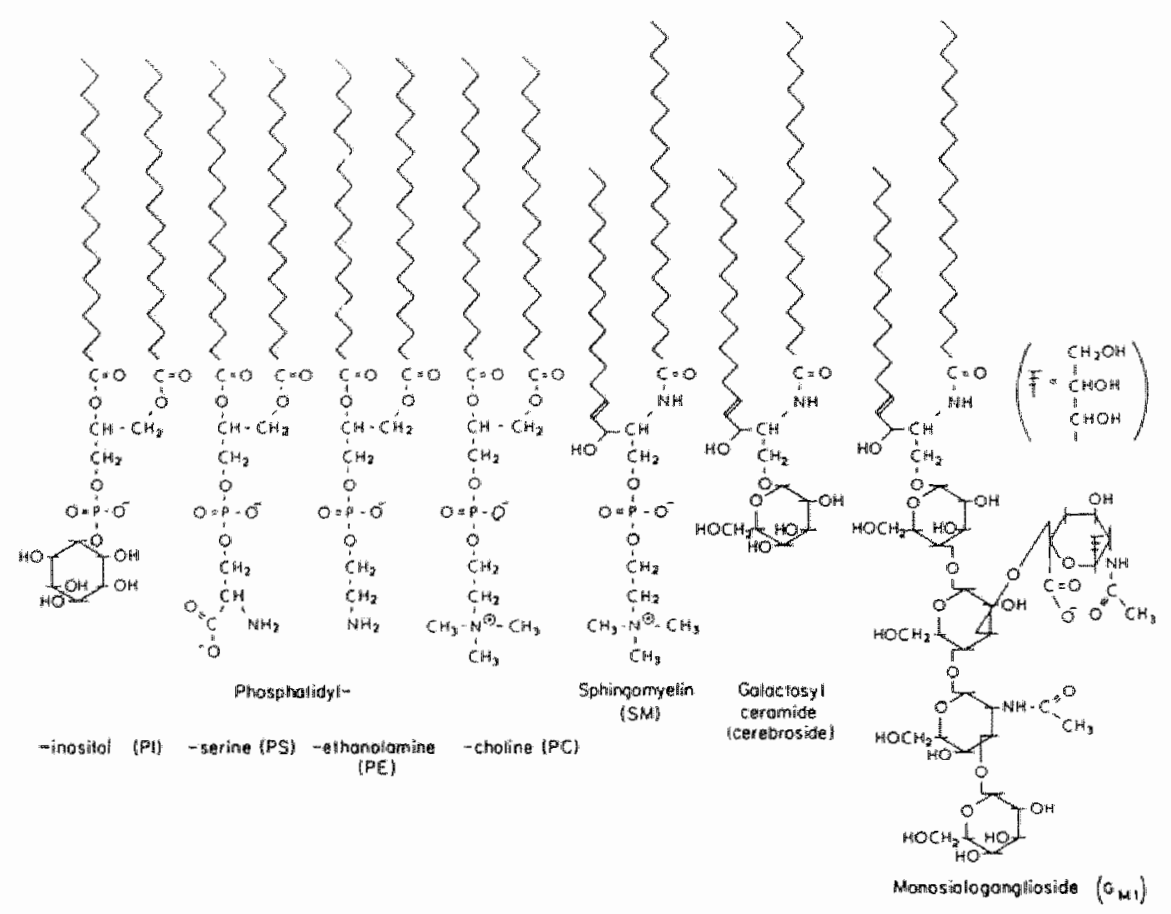

Figute 1.2 General structure of phosphoglycerides (PC, PE, PS, PI). sphingonyetin (SM), cholesterol and wo glycolipids fecrebroside, $G_{m l}$, enthasizing their amphipautic naure and polas head group differentes.

Each type of membrane, however, possesses a variety of lipids (mainly phospholipids, cholesterol and glycosphingolipids, see Fig. 1.2), most of which have properties conducive to lateral compartimentation and formation of distinct domains in the membranes. The composition of phospholipids may also differ considerably between the two hemileaflets of the membrane (vertical compartimentation), as has been demonstrated for erythrocyle and platelet plasma membranes (156, 247, 266, 267), as well as recenlly shown for the myocardial plasma membranes ( 166 ).

Distinct domains of phospholipids may be formed because of differences in headgroup and acyl-chain characteristics and/or by the binding of divalent cations, such as $\mathrm{Ca}^{2+}$ (53). The ligation of $\mathrm{Ca}^{2+}$ to acidic lipid neutralizes the surface charge. 
increases the main lipid transition temperature, decreases the lipid bilayer width, or increases the packing density and order of lipid molecules as indicated by X-ray diffraction and spin- $\forall$ abel techniques $(102,232)$.

The neutral lipid cholesterol may also be involved in the formation of laterally segregated domains, of different fluidity, in biological membranes. As suggested by studies on model phospholipid-cholesterol mixtures, which clearly indicate the presence of cholesterol-rich and cholesterol-poor domains (47.70, 108, 175, 209. 248). It has also been recognized that cholesterol has varying affinities for phospholipids (236) and that cholesterol acts to "buffer" the fluidity of model phospholipids (155). Above the thermal transition temperature of the lipid, cholesterol increases ordering: conversely, cholesterol incorporation below the lipid melting point serves to fluidize the mixed membrane. As most proteins preferentially reside in cholesterol poor domains $(41,107)$, the lateral segregation of cholesterol may also have important actions on the distribution and function of membrane proteins $(157,265)$.

The distribution of carbohydrate, covalently bound to either lipid or protein, is even more asymmetric than that of lipids, since the oligosaccharide side chains of the glycosphingolipids and glycoproteins of both internal and plasma membrames are located on the exoplasmic surface.

The term cell coat or glycocalyx is often used to describe the carbolhydraterich peripheral zone at the surface of most eukaryotic cells (Fig I,3). The glycocalyx of cardiomyocytes is approximately $50 \mathrm{~nm}$ thick (138) and consists of two layers, the surface coat of $\pm 20 \mathrm{~nm}$ (less dense) and the external lamina of $\pm 30 \mathrm{~nm}$ (slightly more dense). The external lamina mainly contains adsotbed glycoproteins. whereas the surface coat is a more integral part of the plasma membrane and contains adsorbed glycoproteins and the oligosaccharide sicle chains of the glycosphingolipids and glycoproteins. The surface coat follows the plasma membrane, as it invaginates 10 form the transwerse T-tubules in cardiomyocytes (78), in contrast to T-tubules in skeletal muscle, which are not lined by an extensive glycocalyx (143). The external lamina and the surface coat are actually two separate layers supposed to be held together by $\mathrm{Ca}^{2+}$-ions (138). It is believed that the glycocalyx is involved in the regulation of ion transport $\left(\mathrm{Ca}^{2+}\right.$ in particular) and excitation-contraction coupling in myacytes (137). 


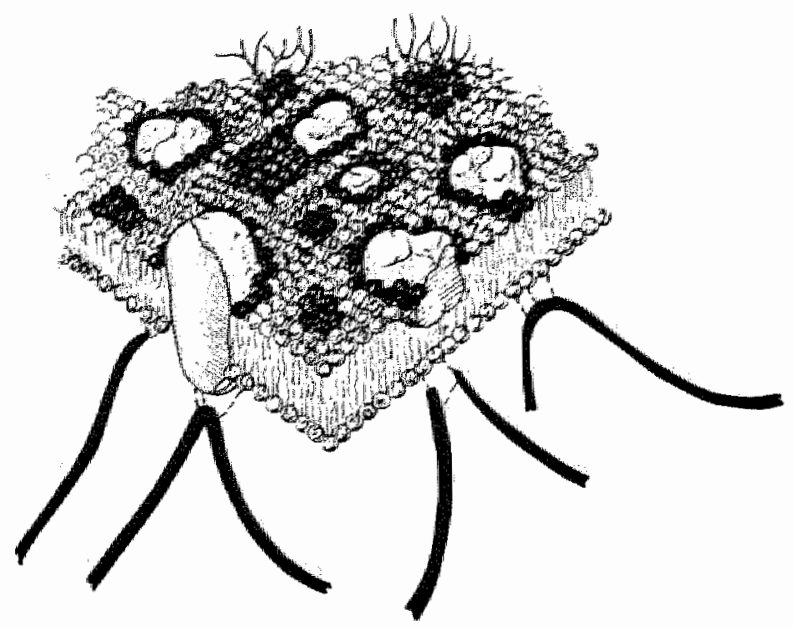

Figure 1.3 The chrrevi wien fie.. "posiffuid mosadc" modell of the plasma membrane. stimntarizing mary points of view louched on this chapfer. The integral nembrane proteins are associaled whin anmular phospholipids, whose head groups are shaded dark in the figure. Ont the upper. exterior face of the membrane. the carbohydrate chairs of the glycoprow reins and glycolipids extend into the mediwn, where they may inferact ahrought sugarmspeciffe interactions witly ofher glycoconjugates ro form a glycocalyx around the cell. in the bulk lipid. patches of different composition segregate to form separate lipid donains. The cyosol face of the mentbrane lacks the glycoconjugates, but has entensive linkages through various membrane componems to the cytoskeleton. The whole is a dynamic structure, each class of componens undergoing differen varieties of morion on different time. scales. fafies: Chriain at al., 1988).

Stability of the inhomogeneous distribution of phospholipids and anchoring of membrane proteins can be achieved by interaction of the membrane with the glycocalyx ol with either of the three classes of cytoskeleton elements: microtubules, microfilaments, and intermediate filaments. The cytoskeleton is known to interact with the plasna membrane, by covalent and non-covalent interactions with both membrane proteins and membrane lipids $(31,113,181)$. These interactions undoubily play impontant roles in mediating several membrane functions and movement of membrane constituents.

The rich structural diversity discussed above means that individual components of membranes will exhibit varying rates of motion. One can therefore no longer speak of "membrane fluidity" in a general sense. The current view on biomembrane structure has lead to a "post-fluid mosaic" model, in which not only membrane lipids and proteins are included, but also the glycocalyx and the cytoskeleton interactions (Fig. 1.3). Regutation of the plasmalemmal integrity is rather complex, since many com- 
ponents (membrane lipids and proteins, glycocalyx and membrane cyloskeletony are involved. These various unteractions have to be taken into consideration when studying the mechanisms of ischemia induced functional and structural changes of the sarcolemma.

1.2.2. Functional changes of the myocardial sarcolemma during ischemia and reperfusion.

A major function of the sarcolemma is 10 act as a selective permeability barrier, a function that is readily hampened by biochemical or physico-chemical changes of membrane lipids during ischemia. Several membrane associated enzymes. controlling $\mathrm{Ca}^{2+}$-fluxes such as $\mathrm{Ca}^{2+}$ ATP'ase, $\left(\mathrm{Na}^{+}, \mathrm{K}^{+}\right)$ATP'ase, $\mathrm{Na}^{+} / \mathrm{Ca}^{2+}$ exchanger and $\mathrm{Na}^{+} / \mathrm{H}^{+}$exchanger, have a decreased activity during ischemia $(14,18,133,217$. 244). This is possibly caused by local alterations of the membrane. Several membrane enzymes are known to be regulated by membrane lipids $(39,182,207,245,262)$, such as boundary (or annular) lipids surrounding integual membrane proteins (see also Fig. 1.3). It has been demonstrated that degradation of phospholipids and accumulation of fatty acids during ischemia, have effects on several ATP"ase activities and $\mathrm{Na}^{+} / \mathrm{Ca}^{2+}$ exchange $(6,186)$. Furthermore it has been demonstrated that lyso-phosphoglycerides exhibit marked effects on membrane integrity in many systems (235). The accumulation of amphipathic lipids, such as fatty acids $(44,167,234,237,242)$, Iysophospholipids (212) and long-chain acylcarnitines (44, 202, 258) may have arrhythmogenic properties in ischemic myocardium $(48,49,51,212)$ and may eventually lead to myocardial damage, as indicated by an enhanced release of enzymes $(27,60)$.

Increased sarcolemmal permeability, as manifested by the release of nacromolecules, such as intracellular enzymes, is one of the characteristic alterations occurring during myocardial ischemia (106). It has long been considered that rupture of the sarcolemma is a prerequisite of this phenomenon (116. 119). However, several studies indicate that other, more subtle, partly reversible membrane alterations are involved in the functional impairment of the sarcolemma during ischemia $(61,161,180,219)$.

Another major function of myocardial plasma membranes is to maintain an electric potential across the membrane and to conduct action potentials. The mem- 
brane potential reflects the contributed diffusion potentials of several ions for which the membrane shows a significant permeability. Since the sodium and calcium ions have a higher concentration outside than inside the cell, they have a strong tenciency to leak into the cell, and must be continually pumped out. The ( $\mathrm{Na}, \mathrm{K}) \mathrm{ATP}$ 'ase of plasma membranes is the enzyme responsible for pumping sodium out of the cell and potassium into the cell against their respective concentration gradients and has a central role in the establishment of electrical potentials along the plasma membrane.

Furthermore the plasma membrane may also be involved in the excitation contraction coupling. Muscle contraction can be triggered by electro-mechanical coupling initiated by depolarization of the plasma membrane leading to an increase in cytoplasmic free $\mathrm{Ca}^{2+}$. The primary role of the sarcoplasmatic reticulum (SR) in the regulation of $\mathrm{Ca}^{2+}$ in skeletal muscle has been generally accepted $(1,79,142)$, and recent studies also indicate that the $S R$ has a similar dominant role in mammalian cardiac (71) and in smooth muscle (213). The regions of the SR (terminal cisternae or junctional SR) serving as the major store of releasable intracellular $\mathrm{Ca}^{2+}$ are separated by a $12-18 \mathrm{~nm}$ gap, transversed by irregularly periodic electron densities, the so-called "feet" or "bridging structures" $(84,216)$. Mitochondria, as is now generally agreed $(37,215)$, do not play a significant role in the micro regulation of cytoplasmic $\mathrm{Ca}^{2+}$. Contraction in most vertebrate skeletal muscle and mammalian cardiac muscle is activated by action potentials that release $\mathrm{Ca}^{2+}$ from the terminal cisternae or the junctional SR. The two dominant mechanisms proposed for the transmission of the message, to release $\mathrm{Ca}^{2}+$ from the plasma membrane to the $\mathrm{SR}$ are, respectively, a conformational change related to movement of electrical charge (38) and the production of a chemical messenger (for example, inositol triphosphate. see 142,214 ).

Myocardial ischemia also induces mitochondrial damage, causing mitochondrial dysfunction and ultrastructural alterations $(8,115,169)$. It is often suggested that mitochondrial function may play a key role in cellular viability during recovery from ischemia. Mitochondrial dysfunction can be restored even after significant nucleotide loss, particularly when the increase in intracellular $\mathrm{Ca}^{2+}$ content can be prevented $(22,72,76,169)$.

There are two fundamental changes related to the structure and function of the 
inner mitochondrial membrane affected by ischemia and relating, in tarm, to most of the changes described thus far $(72,169)$. The first of these is a loss of adenosine nucleotide translocase activity, which may be reversibly inhibited by long chain fatty acyl-CoA esters (202).

A second alteration, contributing to the spectrum of ischemic mitochondrial alterations, is an increase in the permeability of the inner membrane, associated with collapse of the membrane potential and the onset of large amplitude swelling ( 111 . 160). It has been suggested that very limited phospholipid degradation is sufficient to produce a modification of the inner membrane permeability properties (160). Most mitochondrial alterations are considered to be reversible, but restoration of normal mitochondrial structure and function is hampered due to the accumulation of long chain fatty acyl-CoA esters during ischemia and the influx of $\mathrm{Ca}^{2+}$ ions upon reperfusion $(72,76)$. Therefore, mitochondrial alterations are not considered to be the cause of the irreversibility of the cellular deterioration and cell death during the reperfusion period.

Moreover, a defective cell membrane function is generally considered the primary event in the genesis of the irreversible state of ischemic injury (72, 121$)$. The failure in cell volume regulation may be one of the earliest signs of the development of irreversible, ischemia induced myocardial injury. The accumulation of lactate and inorganic phosphate (230) and the development of abnormal myocardial fluid retention during ischemia (100) may lead to an increase in the osmotic load, which in turn affects several myocardial cell functions and may lead to membrane damage and the onset of irreversible cell injury $(114,121,170,222,259)$.

1.2.3. Structural changes of the myocardial sarcolemma during ischemia and reperfiusion.

It is generally accepted that shortage of oxygen supply, as caused by anoxia (73. 110,162$)$, hypoxia $(32,96)$ and ischemia can lead to morphological disorders in myocardial cells. A variety of studies has reported changes at the level of myofibrils. nuclei and mitochondria during myocardial ischemia $(59,76,105,114,115,129,130$. $169,171,183,184)$. 
During the past two decades specific alterations have been observed in the myocardial cell membrane, the sarcolemma. Rupture of the sarcolemma is one of the most clear indications of irreversible membrane damage $(88,116,119)$. The evidence is increasing that more subtle changes are involved in the release of cellular enzymes and the onset of irreversible damage of the sarcolemma $(61,161,180,219)$.

Apart from aggregation of intramembranous particles $(7,8,80,83,164)$, several types of bleb formation have been observed in oxygen deprived cardiac tissue. A variety of "blebs", which likely develop through different mechanisms, has been described. It is therefore of interest to distinguish between different "bleb"-types: (a) glycocalyx blebbing and (b) sarcolemmal bleb-types.

Glycocalyx blebbing occurs when the external lamina loosens from the surface coat and is often (incorrectly) referred to as "pealing of the glycocalyx". This "pealing" of the external lamina is a well documented phenomenon in calcium paradox experiments $(52,79,81,82,105)$ and has also been observed in anoxic hearts $(73,80$. $88,104)$. Divalent cations, such as $\mathrm{Ca}^{2+}$ and $\mathrm{Mg}^{2+}$ may play a role in the pealing of the external lamina (138). Vesiculation of unilamellar vesicles, present between external lamina and surface coat, is sometimes observed as well, in either calcium paradox experiments $(81)$ or anoxic hearts (80).

Sarcolemmal bleb-types can be divided into three major categories: 1) sarcolemmal blebbing, 2) lamellar structures and 3) multilamellar extrusions.

1) Sarcolemmal blebbing most likely accurs after uncoupling of cytoskeleton proteins from membrane proteins and/or lipids (227). The plasma membrane remains intact, but bubbles or blebs are formed by bulging of the lipid-bilayer and these blebs appear to be empty or are filled with either granular cytoplasmic material or mitochondria. Smal! blebs of 1-2 $\mu \mathrm{m}$, called microblebs, are mostly empty and have been observed in anoxic myocytes $(161,162)$. Macroblebs are larger blebs of $25-50 \mu \mathrm{m}$ and are mostly filled with mitochondria. They have been observed in hearts after anoxia $(22.162)$ or after metabolic inhibition $(29,45)$.

2) Lamellar structures are bilayered structures in the form of irregular vesicles with widely spaced, concentrically arranged lamellae, with variable interlamellar distances. Lamellar structures have been described in ischemic or anoxic myocardium as fragmentary membranes in vacuoles (168) or as circular membrane vesicles often 
referred to as subsarcolemmal blebs $(179,221)$. However, this type of lamellar structures can also be induced by inappropriate fixation of too large pieces of tissue (95). Small unilamellar vesicles, just beneath the cell membrane, should not be referred to as subsarcolemmal blebs, since they are most likely generated by vesiculation of the membranes due to penetration of fixative into the cells (197).

3) Multilamellar structures are tightly packed, concentric, multi-layered membrane structures with a constant lamellar repeat distance. As has been indicated by freezefracture electron microscopy. multilamellar vesicles are likely to contain pure lipid material, since the fracture faces of liposomal structures are found to be free of intramembranous particles $(21,164,250)$. Multilamellar vesicles have been observed under various experimental conditions in several tissues. Their occurrence in hearts as well as in other tissues will be discussed in more detail.

\subsection{MULTILAMELLAR STRUCTURES}

\subsubsection{Multilamellar structures in non-cardiac tissues.}

A well known example of multilamellar structures are the "inclusion bodies". also called "lamellar bodies", in type II pneumocytes of lung tissue $(63,208)$. These secretory bodies produce the surfactant secretion and are known to contain a high concentration of saturated phospholipid $(62,94,141)$. These compounds are to a large extent extracted during routine processing of tissue for electron microscopy. This is the major reason why preservation of secretory bodies without the induction of artifacts has rarely been achieved. The electron microscopic appearance (homogenous or lamellar) of these structures can differ clepending on the preparative methods employed $(15,112,135,226)$.

Chemicals like tannic acid have been suggested as additives to aldehyde fixatives and used to improve the ultrastructural preservation of these secretory bodies $(12$. 124). Tannic acid has also been employed to improve the delineation of cellular membranes $(178,205,252)$. However, several studies have revealed that the presence of multilamellar structures depends on treatment with tannic acid $(112,178)$ or may 
actually be considered antificial structures induced by tanaic acid treatment (251).

A rather unusual interpretation of the use of tarnic acid has been made by Blanchette-Mackie and co-workers, who considered the appearance of multilamellar structures after tannic acid treatment of adipose or heart tissue to reftect lipolytic activity, still proceeding after aldehyde fixation, and fatty acid transport over membranes $(19-21,256)$.

\subsubsection{Multilamellar structures in cardiac tissue under "normal" conditions.}

Multilamellar structures develop in myocytes of glutaraldehyde-fixed rat hearts when the tissue is incubated with tannic acid (pH 7.4) at $25^{\circ} \mathrm{C}(256)$. These investiga* tors concluded that the multilamellar structures were composed of farty acids formed by lipolysis, since the number of lipid droplets decreased during incubation. In myocytes these multilamellar vesicles were mostly observed adjoining lipid droplets or mitochondria. They noted that tightly packed multilamellar structures did not develop in the absence of tannic acid and suggested that tannic acid might produce changes at hydrophilic surfaces of lipid bilayer's resulting in tight packing (by dehydration) of lamellar structures.

\subsubsection{Multilamellar structures in cardiac tissue after ischemia and reperfusion.}

Multilamellar vesicles have been reported in ischemic and reperfused isolated rabbit hearts (164). Hearts subjected to 90 minutes of ischemia showed aggregation of intramembranous particles in the sarcolemma and extrusion of liposomal structures from mitochondria. Reperfusion after 90 minutes of ischemia resulted in further aggregation of the intramembranous particles and disnuption of the sarcolemma, which was evidenced by the extrusion of liposomal structures from the sarcolemma and loss of contiruity of the bilayer. Tannic acid was thought to be necessary to retrieve multilamellar structures with thin-section electron microscopy. Direct ultra-fast freezing and fracture replication and conventional freeze-fracture electron microscopy (with primary fixation, using a combination of glutaraldehyde and tannic acid) has also been applied. The authors reported that identical results were obtained with both 
techniques. but the most convincing micrographs presenting the multilamellar appearance of the extrusions were obtained from conventional freeze-fracture electron microscopy, using tannic acid based fixation. which was also the case in later studies (165).

\subsection{ALTERATIONS IN MYOCARDIAL (LIPID) METABOLISM}

\subsubsection{Alterations in myocardial (lipid) metabolism during ischemia.}

A variety of metabolic changes, leading to functional and structural alterations and directly or indirectly affecting myocardial membrane integrity, is associated with myocardial ischemia. The cellular content of creatine phosphate falls rapidly and precedes the progressive decline in total ATP content during myocardial ischemia (233). Depletion of glycogen and accumulation of low molecular weight substances. such as lactate. inorganic phosphate and hydrogen ions readily occurs and may add to an osmolar load (230). These initial changes during myocardial ischemia will not immediately cause irreversible cell injury. It is generally believed that sarcolemmal damage is the proximate cause of irreversible myocardial cell injury $(72,121)$.

Several mechanistic explanations have been given for the metabolic alterations leading to membrane destabilization during myocardial ischemia. Firstly, the myocardial energy state (creatine phosphate. ATP) diminishes during ischemia $(116,221,234)$. Depletion of ATP below a crilical level leads to inhibited activity of ionic pumps. for instance causing acidosis (149) and disturbed $\mathrm{Ca}^{2+}$ homeostasis (148). A diminished ATP level aiso affects the interaction between membranes and the cytoskeleton (116. 121) on its turn leading to structural membrane alterations.

Secondly, massive influx of $\mathrm{Ca}^{2+}$ has been associated with mitochondrial swelling (184) and contracture band necrosis $(76,89,114,200)$. Short periods of ischemia leaves myocytes structurally intact and do not lead to accumulation of $\mathrm{Ca}^{2+}$ when blood flow is restored (118). Interestingly, above cytosolic $\mathrm{Ca}^{2+}$ levels of $3 \mu \mathrm{M}$, anoxic cardiomyocytes do not recover by reoxygenation (1). This may be because the setpoint for $\mathrm{Ca}^{2+}$ accumulation by milochondria has been transgressed. Above this threshold, 
mitochondria spend respiratory energy predominantly for $\mathrm{Ca}^{2+}$ uptake instead of providing ATP (36).

Changes in the ionic environment, such as accumulation of $\mathrm{Ca}^{2+}$ and $\mathrm{H}^{+}(149)$. after longer periods of ischemia may alter the physico-chemical properties of membrane lipids. Thus, influencing the functional properties of membranes and probably causing phase separation of membrane lipids, possibly associated with lipidic extrusions (250). On the other hand, elevated levels of $\mathrm{Ca}^{2+}$ in the ischemic myocardium may enhance phospholipase activity $(72,254)$. For instance, membrane assochated phospholipase $\mathrm{A}_{2}$ activity, with substrate specificity for phosphatidylcholine and phosphatidylethanolamine, has been found to be markedly increased in hypoxic myocardium (128).

Thirdly, as a consequence of increased phospholipase activity. membrane phospholipids are prone to degradation $(16,87,254,260)$. Alternatively, resynthesis of phospholipids $(44,234)$ has been suggested to be inhibited by the blocking of acyl-CoA synthetase by AMP and adenosine, which are accumulating in the myocardium during ischemia. The decrease in ATP. which is necessary as co-substrate in the resynthesis of phospholipids, as observed during myocardial ischemir plays most likely a minor role $(233,234)$. Alternatively, membrane damage, like fracturing of the sarcolemma, might be caused by physical forces, such as intracellular edema or contracture induced mechanical stress. As a consequence, enhanced phospholipid degradation may also be an epiphenomenon reflecting autolysis of already irreversibly clamaged cells (242).

Fourthly, degradation of phospholipids will lead to an increased level of lysophospholipids $(49,212,221)$ and fatty acids $(44,234,237,240)$, amphipathic compounds that will cause functional and structural alterations when intercalated with membranes (50, 127). Several investigators have reported accumulation of long chain acylcamitines and long chain acyl-COA $(203,258)$, for which similar effects on membrane function lave been proposed (48).

In recent years there has been increasing evidence that alterations in myocardial lipid metabolism play an important role in myocardial membrane dysfunction and, hence. in the pathogenesis of ischemic cell injury. The basis of this dysfunction is not yet clear. alterations in membrane phospholipid content and composition, and the generation of amphipathic metabolites, which can directly affect the integrity of the 
membrane lipid bilayer, are likely to be the important factors involved. If or how these phenomena may initiate structural nembrane alterations is still subject of investigation.

1.4.2. Alterations in myocardial (lipid) metabolism during reperfusion.

It is well accepted that the ischemic myocardium cannot recover without restoration of the blood flow $(131,184)$. It has been demonstrated that reperfusion after short periods of myocardial ischemia indeed results in complete recovery $(26$. 117) but after prolonged duration of ischemia hearts do not recover upon reperfusion or may even suffer from intensified damage $(33,77,117,120,184,200)$.

During reperfusion oxygen-derived free radicals can be formed $(4,97,253,254)$ leading to further deterioration of plasmamembranes. Lipid peroxidation of the structural important poly-unsaturated fatty-acyl chains of phospholipids affects the "fluidity" of the membrane and thus hamper its primary action as permeability barrier. Consequently the osmotic load may increase associated with a massive influx of $\mathrm{Ca}^{2+}(200,228)$, on its tum initiating enhanced activity of proteases and phospholipases (42, 54, 101, 144). Hence, reperfusion may add to enhanced myocardial cell damage, rather than recovery $(77,239)$.

Recently, it has been demonstrated that reperfusion results in additional changes in myocardial lipid homeostasis in rats $(233,234)$. Furthermore, the additional rise in fatty acids during reperfusion was found to be positively correlated witl the cumulative leakage of lactate dehydrogenase. These findings indicate that repertusion induces additional alterations in myocardial lipid metabolism which may be associated with additional membrane dysfunction and myocardial cell injury. It needs further investigation to reveal which structural membrane alterations are associated with metabolic alteration during ischemia and reperfusion.

\subsection{FIXATION OF TISSUE LIPIDS}

The main objectives of fixation (for electron microscopy) are to preserve the 
structure of cells with minimum alteration from the living state with regard to wollume, morphology, and spatial relationships of organelles and macromolecules. minimal loss or relocation of tissue constituents. and protection of specimens against subsequent treatments including dehydration, embedding. staining, and exposure to the electron beam in the high vacuum. It was originally observed that lipids were poorly fixed by many fixation protocols, as excessive extraction appears to take place during dehydration and embedding (224). This puts a severe constraint on devising schedules for tissue preparation, especially when ultrastructural examination of lipid structures is intended. Alternative methods with minimal lipid extraction make use of fixation followed by freezing and freeze-substitution or by ultra-fast freezing without chemical fixation (freeze-arresu/ physical fixation). Ultra-fast freezing takes in the order of $10^{-5}$ seconds and is therefore extremely suitable for studying fast processes, such as the release of neurotransmittors and fusion-events of biological membranes, or when redistribution of lipids or lions has to be prevented. But, since redistribution of lipids will not influence the ultrastructure of most tissues, physical fixation by fast freezing is not generally applied in morphological studies. Most ulitrastructural studies still relly on chemical fixation of cell and tissue components.

Among the chemical fixatives aldehydes and osmium tetroxide are the most commonly used. Fixation and retention of phospholipids during lissue processing for electron microscopy does not only depend on the chemical composition of the phospholipid polar head groups (e.g. serine and ethanolamine are partly fixed by glutaraldehyde) and the extent of unsaturation of the fatty acid chains (fixed by osmium tetroxide) $(178,224)$. but also on the presence of structural links between phospholipids and proteins and on their spatial distributions.

Glutaraldethyde, the most commonly used fixative since its introduction by Sabatini (177), is a 5 carbon dialdehyde. Originally it has been proposed that glutaraldehyde undergoes aldol condensation in aqueous solutions to form condensation polymers. The reaction of primary amine groups in proteins with the carboxyl carbon. of these condensates gives rise to $\alpha \beta$-unsaturated Schiff bases, which are insoluble in the protein matrix (172). The aldol condensates permit many sites for crosslinking. More recently, it has been shown that pyridines and pyridine polymers are produced from glutaraldehyde-amine precursors $(99,122)$. Therefore, it has been proposed that 
these pyridine co-polymers are the more important cross-linking elements which bridge erratically spaced primary amino groups in cells (122). Glutaraldehyde interacts with phospholipids in an analogous fashion by cross-linking at primary amine groups. Thus. phosphatidylethanolamine and phosphatidylserine are cross-linked by glutaraldehyde whereas phosphatidylcholine and phosphatidylinositol are not because they lack primary amines $(92,123,261)$.

Phosphatidylcholine is generally the main constituent of the membrane lipid bilayer. whereas aminolipids (PE, PS) and cholesterol are present in a lesser amount, which might lead to spatial separation of these lipids aggravating cross-linking by glutaraldehyde. By the addition of diamines to the glutaraldehyde fixative, threedimensional structures of variable size, configuration and charge displayment are formed $(24,25)$, enabling the fixation of aminolipids and proteins, separated over greater distances.

Osmium tetroxide interacts with phospholipids by cross-linking at fatty acyl double bonds $(103,173,225)$. Therefore, it is apparent that saturated glycerophosphatides, lacking primary amine groups (such as PC, PI) are not cross-linked by either aldehyde or osmium tetroxide. Thus, they are prone to dissolve and diffuse out of the tissue during conventional dehydration and embedding (134, 178).

Several additives to aldehyde or osmium fixatives, as well as alternative preparation protocols, have been suggested and applied to improve the preservation of tissue lipids. A brief overview will be given and possible reaction mechanisms, as well as the advantages and limitations will be discussed in more detail.

One of these additives is tannic acid, it is particularly known for its industrial application in cotton and leather production. Commercial tannic acids are natural products, gallotannins with a complex composition of polyphenolic material, mostly with a similar galloylated glucose structure (Fig, 1.4). The degree of glucose esterification by phenolcarboxyl acids, such as gallic, digallic or trigallic acid, varies depending on the natural source. The most effective for the preservation of structures, contrast enhancement and penetration are low molecular weight galloyl glucoses, when applied between osmication and dehydration (205). The mechanisms of tannic acid reactions seem to be complex. One of the best described actions of tannic acid is the "mordanting" effect of tannic acid on osmicated structures, causing enhanced 

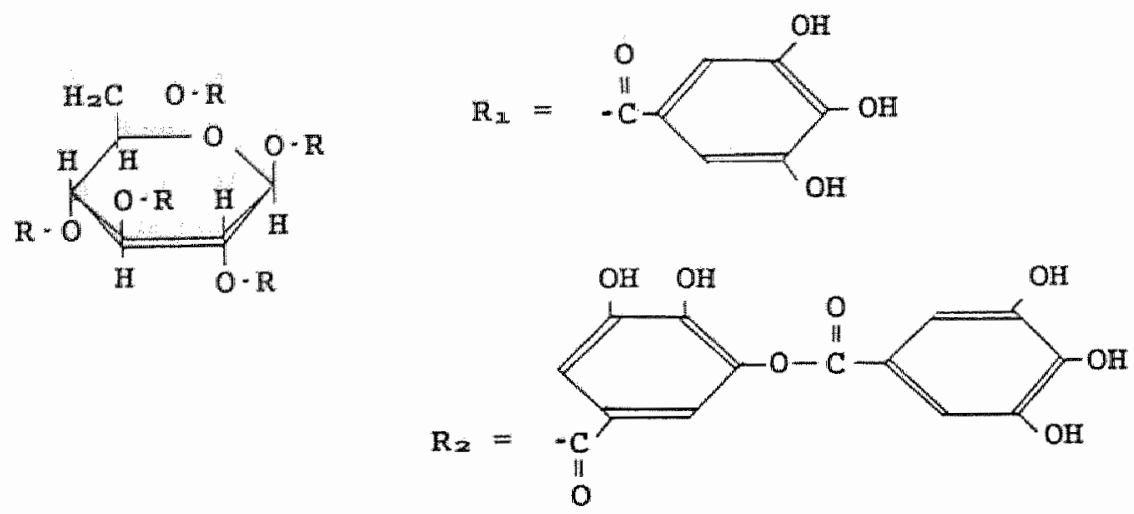

Figure 1.4 Siruchure formula(s) of tonstic acid emphasizing the galloyiared glucose structure, which nost tamic acids have in common. The ghincose backbone may be esteriffed with phenolcarbaxyl acids. whith as gallic $\left(R_{1}\right)$, digallic $\left(R_{2}\right)$ on trigallic acid, varying from one natural source to another.

contrast in the final section. Which structures are mordanted seems to depend on the stage at which tannic acid is applied $(205,252)$. Tannic acid has been suggested as an additive during fixation to improve the retention of saturated phosphatidylcholines in tissue preparations $(134,178)$. Since it has been shown to interact with the choline headgroup of glycerophosphatides, resulting in a complex that becomes further stabilized by subsequent osmication (125).

Uranyl acetate may be used to retain phospholipids in cells, particularly in cell membranes where increased contrast is obtained (28). It has been shown to reduce phospholipid phosphorus extraction (204). Uranyl acetate when used as "en-bloc staining", has therefore also been considered as tertiary fixative (46).

The addition of calcium ions to fixatives, in concentrations of $1-3 \mathrm{mM}$. has been recommended for the preservation of lipids (95). This recommendation has been based on the improved stability of membranes, resulting in fewer myelin-like structures $(30,95)$. Biochemical data suggested that divalent cations, particularly $\mathrm{Ca}^{2+}$ and $\mathrm{Mg}^{2+}$. seem to control the stability of erythrocyte membranes by the formation of a 
tertiary complex between the anionic groups of a protein and a phospholipid molecule (66, 176). It has been suggested that the addition of calcium ions is particular beneficial for the preservation of mitochondria $(30,95)$.

The use of potassium ferrocyanide in fixation was recommended by Elbers et al. (68) in a study of "tri-complex" fixation of phospholipids. It was therefore originally suggested to be of particular value in preserving phospholipids and hence, in improving the fixation of membranes (126). It has also been shown to improve the overall retention of lipids (55). However, the advantages of tri-complex fixation has been subject to criticism (93). The principle of ferrocyanide-osmium is likely based on osmate $\left(\mathrm{Os}_{6}{ }^{2-}\right)$ formation (151), but regarding the precise reaction mechanisms contradictory ideas have been proposed $(56,257)$. Although the considerable doubt to the benefit of the method has grown in years, De Bruijn and co-workers have been advocating tri-complex fixation for many years (56-58).

Imidazole-buffered osmium tetroxide has been shown to enhance the penetration of osmium-imidazole and to improve staining of unsaturated lipids, suggesting that imidazole facilitates the interaction of osmium tetroxide with unsaturated lipids in tissue (3).

It has also been attempted to minimize lipid extraction by using polar dehydrating agents, such as Durcupan and dioxane (187), and glycol methacrylaat (63), with only moderate success. Dehydration with acetone instead of ethanol seemed, to some extent, to reduce the extraction of lipids $(9,98)$.

An alternative approach to the preservation of phospholipids makes use of low temperature techniques and renders chemical treatment unnecessary when frozen specimens only have to be depicted and not have to be used, for instance, for autoradiography. This approach guarantees almost complete retention of all phospholipid species (249. 255). However "even if gross extraction of phospholipids is prevented by using freeze-substitution and low temperature embedding (which likely holds for the chemical methods described above as well), the chance on redistribution remains. However, as mentioned earlier this may be of less concern when only morphologic studies are intended.

The range of fixatives, additives and alternative methods available for processing tissue for electron microscopy with the aim to prevent relocation and extraction of 
(phospho)lipids is extensive, making the choice of the technique to be used difficult. It has to be borne in mind that very often a compromise has to be made, especially when more than one technique has to be carried out on the same small sample, as in biopsy materials.

\subsection{AIM OF THE STUDY}

The aim of this study was to investigate ultrastructural membrane alterations, as induced during the early phases of ischemia and after subsequent reperfusion. Since various membrane components, like glycocalyx, cytoskeleton and membrane lipids and proteins, may be involved in ischemic membrane alterations special attention was paid to these components.

\subsubsection{Glycocalyx alterations.}

Attempis were made to improve the preservation of cardiac membranes in general, and membrane lipids and proteins, glycocalyx and the membrane associated cytoskeleton elements specifically. Most (conventional) fixation and preparation protocols for electron microscopy only consider the preservation of the separate components. An exception seemed to be a fixation-method with glutaraldehyde in combination with diamines, such as lysine. This method was found to improve the preservation of both the glycocalyx and cytoskeleton proteins $(24,25)$.

We have demonstrated that perfusion fixation with a combination of (2\%) glutaraldehyde and ( $50 \mathrm{mM}$ ) lysine, in a slightly modified way as originally proposed by Boyles, is suitable for studying cardiac membranes (188). This fixation method gave a fine delineation of the sarcolemma and was found to improve the contrast of the glycocalyx in a more homogeneous way and with less precipitates than with (1\%) tannic acid treatment. Tannic acid treatment was conducted after osmication but before dehydration of $(2.5 \%)$ glutaraldehyde fixed cardiac tissue, as suggested by Simionescu and Simionescu (205). No alterations of the glycocalyx were observed in both Wistar-Kyoto and spontaneously hypertensive rats after 45 minutes of global 
ischemia with or without reperfusion. Since leakage of cellular components can be observed under these conditions it was concluded that an intact glycocalyx adhering to the sarcolemma is not an indication of membrane integrity (188).

\subsubsection{Alterations in membrane lipids.}

Our major aim was to investigate early ischemia induced alterations in the membrane lipid architecture, with emphasis on the formation of multilamellar extrusions. To this end it was explored whether perfusion fixation with gitutaraldehyde and lysine, in combination witl partial dehydration and embedding in LR White resin, was appropriate for the preservation of multilamellar extrusions. Several lamellar structures, but not in the sense of true multilamellar vesicles (see definition in chapter 1.2.3) were found in both ischemic and reperfused rat myocardium. These lamellated structures were predominantly observed intracelluilarly, but not in contact with the sarcolemma or mitochondrial membranes (192).

As mentioned earlier, it has been documented that multilamellar vesicles can be observed with thin-sectioning electron microscopy with the use of tannic acid during fixation (164). Because, tannic acid has a complex and variable composition and little is known about the reaction mechanism(s) of tannic acid with phospholipids, we have tried to gain more insight into the interaction(s) of tannic acid with phospholipids and the use of tannic acid based fixation in lissue preparation.

In chapter 2 the dual effect of tannic acid on the preservation and ultrastructure of phosphatidylcholine vesicles is discussed. This dual elfect of tannic acid concems a reduced extraction of phosphatidylcholine from unilamellar vesicles and aggregation and formation of multilamellar structures.

In chapter 3 a more detailed study on tannic acid induced vesicle aggregation. fusion and the formation of multilamellar vesicles is presented. In this study a variety of techniques have been applied to investigate the effects of tannic acid on small unilamellar vesicles, specifically containing phosphatidylcholine. Based on the results obtained from the experiments described in chapters 2 and 3 we tend to consider the use of tannic acid based fixation as a "cytochemical" method to demonstrate instability of membrane lipids, as reflected by the "secondary" induction 
of multilamellar vesicles. In this light we have used tannic acid based fixation to examine ischemia and reperfusion induced membrane destabilization in isolated rat and rabbit hearts.

In chapter 4 the influence of reperfusion and the duration of ischemia on the formation of multilamellar extrusions in isolated rabbit hearts is presented. The time course, in which multilamellar extrusions become apparent, was studied. In addition it was investigated whether differences exist in shape and/or (sub-jcellular localization of multilamellar vesicles in cardiomyocytes. A comparison was made with the degree of ischemic injury, as scored on a semi-quantitative scale, based on several morphological criteria.

Chapter 5 deals with the correlation in time between the increase in the number of multilamellar vesicles and the metabolic alterations, with emphasis on lipid metabolism and the energy state. These experiments were performed on isolated, ischemic and reperfused rabbit hearts. Special attention was paid to lipid metabolism because there is increasing evidence that alterations in lipid metabolism are involved in ischemia induced membrane changes.

In chapter 6 a comparison is made between rat and rabbit hearts, regarding the formation of multilamellar vesicles and metabolic alterations. The choice of animal species was based on the finding in the "in vitro" experiments, as presented in chapter 3, in which phospolipids extracted from rabbit and rat hearts were found to react with tannic acid in a different way. This difference in reaction was thought to be based on the differential lipid composition of rat and rabbit cardiac membranes.

Chapter 7 provides a general discussion of the results obtained, focusing on the possible mechanisms involved in the reaction of tannic acid with phospholipids and the possible use of multilamellar vesicles to quantitate ischemia and reperfusion induced myocardial (membrane) injury.

\subsection{EXPERIMENTAL APPROACH}

The effect(s) of tannic acid on phospholipid preservation and ultrastructure during processing for electron microscopy were studied in a model system, using 
sonicated small unilamellar phospholipid vesicles. The interactions of tannic acid with various vesicle-preparations (containing different phospholipids or combinations of phospholipids) were monitored using a variety of approaches such as scintillation spectrophotometry, turbidimetry, thin-section electron microscopy, cryo-electron microscopy of vitrified thin films and freeze-fracture electron microscopy (chapters 2 and 3 ).

The effect(s) of reperfusion and the duration of ischemia on multilamellar extrusions in cardiomyocytes was studied in isolated rabbit and rat hearts (chapter 4 . 5 and 6). In rabbit hearts (chapter 4 and 5) the duration of ischemia was varied ( 30. 60 and 90 minutes) and a subset of ischemic hearts was reperfused for 30 minutes (perfusate was collected to establish the cumulative amount of enzyme released during the period of reperfusion). At the end of the experiments biopsies were taken from the apical side, freeze-clamped and used for biochemical analysis (chapter 5). The remainder of the hearts were immediately fixed by perfusion with a combination of glutaraldehyde and tannic acid and further processed for electron microscopic and morphometric examination (chapter 4). Detailed descriptions of the materials and methods involved is found in the methods section of the aforementioned chapters. 

CHAPTER 2

DUAL EFFECT OF TANNIC ACID ON THE PRESERVATION AND ULTRASTRUCTURE OF PHOSPHATIDYLCHOLINE VESICLES

Based on: A.H.G.J. Schrijvers, P.M. Frederik, M.C.A. Stuart, G.J. Van der Vusse, R.S. Reneman (1989) Mol Cell Biochem 88: $91-96$. 


\section{INTRODUCTION}

Reliable fixation of phospholipids is difficult to achieve, since most lipids react poorly with commonly used fixatives for electron microscopy. Aldehyde fixatives react with amine groups of phosphatidylserine and phosphatidylethanolamine but not with phosphatidylcholine, while osmium tetroxide fixes phospholipids by addition to double bounds of unsaturated fatty acids.

It is demonstrated that tannic acid interacts with the choline headgroup of phosphatidylcholine, when added to aldehyde fixative and the resulting complex is stabilized by post-fixation with osmium tetroxide (125).

In this context the use of tannic acid has been advocated for improving the preservation of the lipid contents of the secretory bodies of type 11 pneumocytes in long issue (12, 124). These secretory bodies are known to contain a high proportion of saturated phosphatidylcholines $(75,98,141)$, compounds which are extracted to a large extend during routine processing of tissue for electron microscopy. The ultrastructure of these secretory bodies, also named "inclusion bodies" or "lamellar bodies", varies with the method of tissue preparation $(98,112,226)$. Structures composed of tightly packed concentric lamellae, as found in the lung, are also found in other lissues when treated with tannic acid. In ischemic myocardium such lamellar vesicles are retrieved in thin-sections as extrusions from mitochondrial and sarcolemmall membranes (164, 189).

The way in which tannic acid acts in tissue preparation is incompletely understood, partly because tannic acid is a natural product with a complex and variable composition. Its way of action is sometimes referred to as "fixation" (124, 145). "staining" (229) or "mordanting" (125, 205, 252). In the latter case tannic acid is considered as a mordant between osmicated structures in tisste and lead stains. Thus, tannic acid can be used lo increase the contrast in tissues by its mordanting effect. The structures or substructures that are affected by the mordant depend on the stage at which tannic acid is introduced in the hixation-schedule $(205,252)$.

These findings raise the question as to whether lamellar structures in tissues are present in the living-state or are induced by the treatment with tannic acid. We will report on the mode of action of tannic acid in preserving phospholipid vesicles 
through processing and in the induction multilamellar structures. A model system is used of phospholipid suspensions, sonicated to form small unilamellar vesicles (SUV's). These phospholipid vesicles are examined in the ellectron microscope ether "directly" by vitrification (cryo-electron microscopy) of by chemical fixation (with and without tannic acid) followed by dehydration and embedding.

\subsection{EXPERIMENTAL PROCEDURES}

Small unilamellar vesicles (SUV's) are made from either dimymistoylphosphatidylcholine (DMPC) or dioleoylphosphatidylcholine (DOPC) (synthetic phospholipids purchased from Sigma P0888 and P1013, respectively). To this end the phospholipids are dissolved in chloroform/methanol $(9 / 1)$ and are dried under a stream of dry $\mathrm{N}_{2}$-gas and the lipid residue is suspended in phosphate buffered saline (PBS; 20 $\mathrm{mM}, \mathrm{pH}$ 7.2) in a final concentration of $5 \mathrm{mg}$ phospholipid/ $\mathrm{ml}$. The phospholipid suspension is sonicated until a clear to slightly opalescent suspension is obtained.

"Direct" examination of the SUV-preparations is accomplished by a cryo-electron microscopic technique, as described in detail in chapter 3.2.4.

To investigate the effects of tannic acid on the preservation of phosphatidylcholines and the ultrastructural appearance of the SUV"s when glutaraldehyde fixation is used, several treatments are carried out each procedure followed by post-fixalion and further prepared, as described in detail in chapter 3.2.6.

To examine the effect of tannic acid on the extraction of phosphatidylcholine during processing, a SUV-preparation is made containing DMPC with a tracer of ${ }^{14} \mathrm{C}$-DMPC. ${ }^{14} \mathrm{C}$-DMPC containing SUV's are treated for 10 minutes with either $2.5 \%$ glutaraldehyde or $2 \%$ tannic acid or with a combination of both substances. Aggregates are induced, by the addition of tannic acid, and cab be easilly pelleted by shont centrifugation. The amount of radioactivity. removed from the aggregates (as measured in the supernatant) during each processing step, is assessed by liquid scintillation spectrometry. A scintillation spectrometer equipped with a quenchmeasurement program is used for all determinations. Counting efficiencies range from $40.45 \%$ to $90.75 \%$. All data are reported in terms of dpm corrected for efficiency. 

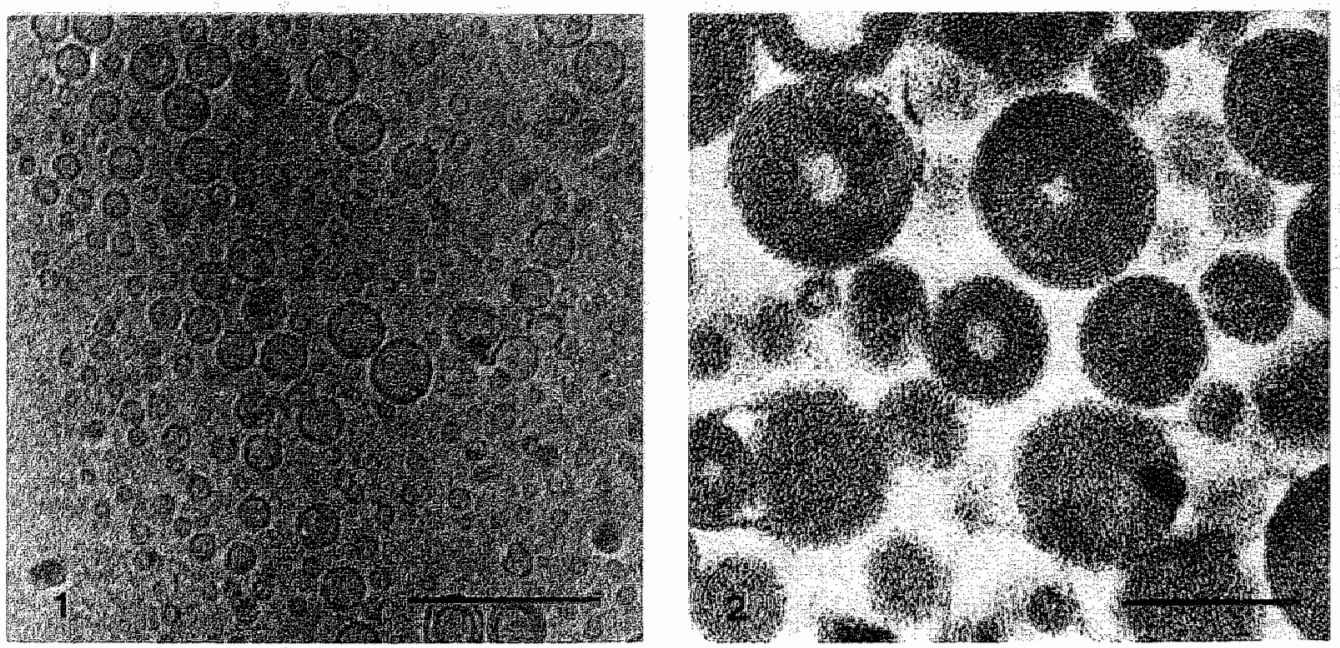

Figure 2.1 DMPC-SUV's "directly" observed by cryouelectron microscopy. The smallest vesicles hone a dianneter of $40 \mathrm{~mm}$. The bar represents: $0.2 \mathrm{~km}$.

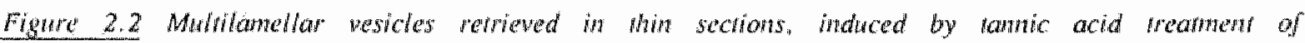
DMPC-SUV's. The distance behween the lamellae has a periodicity of about 5 mm. Trealnent with anmic action ing glaraldehyde has a similar result. The bar represents 0.2 unn.

\subsection{RESULTS}

The vitrified thin films made from vesicle suspensions, prepared by sonicating dimyristoylphosphatidylcholine (DMPC) or dioleoylphosphatidylcholine (DOPC) show small unilamellar vesicles when observed by cryo-electron microscopy (Fig. 2.1). Fixation of DMPC-SUV's with glutaraldehyde in combination with post-fixation by osmitum tetroxide results in the extraction of phospholipid during dehydration and no material is retrieved in the Epon. Direct fixation of DMPC-SUV"s with osmium tetroxide provided the same result. When DMPC-SUV's are fixed with tannic acid with or without glutaraldehyde rapid aggregation takes place (within one second) resulting in clouding of the suspension and the subsequent formation of a white sediment. This sediment, when post-fixed with osmium tetroxide can be embedded in Epon and the material is retrieved in thin sections as multilamellar vesicles (MLV's) with a period- 

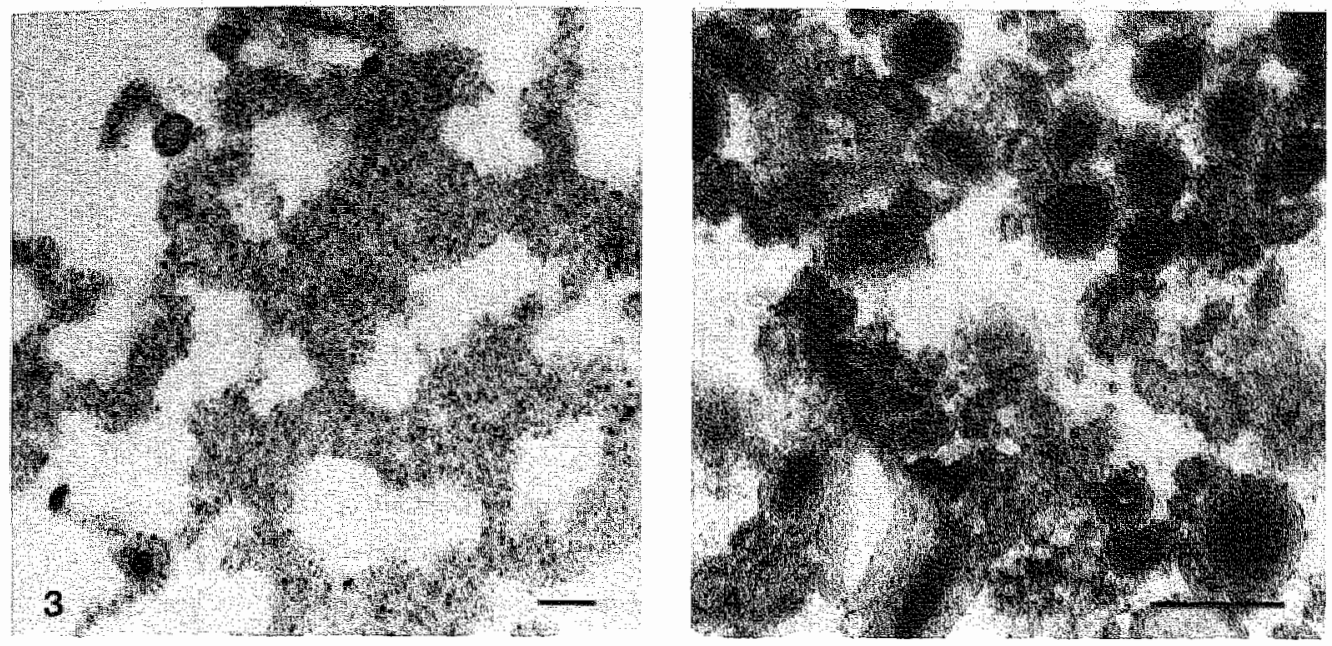

Figure 2.3 Ansorphons strwchures found in thin sections afier ghataldehyde and osminu tetrowide post-fixution of DOPC.SUV's. The bar represens 0.2 un.

Figtre 2.4 Tanuic acid induced jomation of multilanellar vesicles from DOPC-SUV's. The periodicity is abou 6 wh. Treatmen with ramnic acid in glutaraldehyde has a similar result. The bar represests $0.2 \mu m$.

icity of approximate $5 \mathrm{~nm}$ (Fig. 2.2). When post-fixation with asmium tetroxide is omitted the phospholipid is extracted during the acetone dehydration steps.

To investigate cooperative effects of tannic acid and osmium tetroxide on the acylchains of the phospholipids. SUV-preparations containing dioleoylphosphatidylcholine (DOPC) are made. The fixation of DOPC SUV's by osmium tetroxide or by glutaraldehyde followed by osmium tetroxide results in the preservation of amorphous structures (Fig. 2.3). Fixation of DOPC-SUV's with tannic acid in glutaraldehyde or tannic acid alone results in quick aggregation, leading to MLW's found in thin sections with a periodicity of about $6 \mathrm{~nm}$ (Fig. 2.4). When a higher tannic acid concentration is used MLV's aggregate to form multilamellar sheets (Fig. 2.5). "The periodicity (lamellar repeat distance) of the multilamellar structures is not inlluenced by the concentration of glutaraldehyde or lannic acid. The number of MLV's and their size are reduced by lowering the tannic acid concentration. 


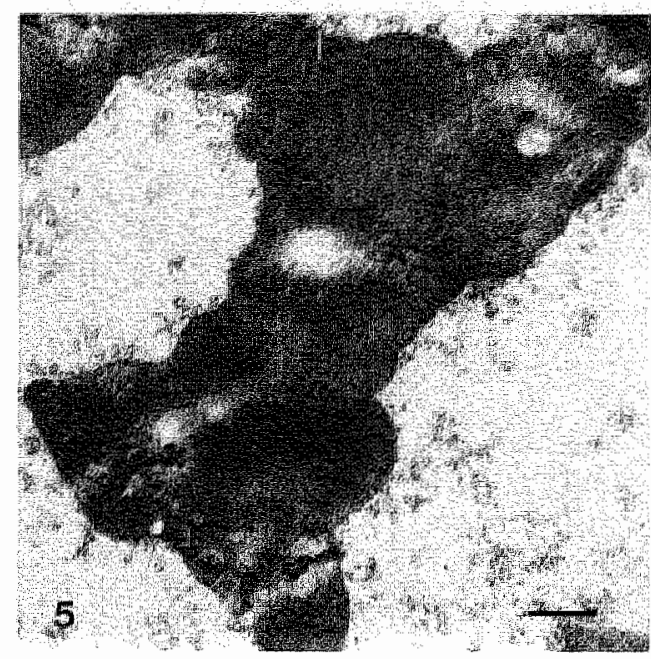

Figure 2.5 Mutrilamellar sheets found in thin sechons affer meatment of DOPC-SUV's with a 5 times higher concentration of rannic acid in gituraldehyda. The bar represerns 0.2 pan.

Tannic acid prevents extraction of DMPC during processing of DMPC-SUV's (Table 2.1). There is no difference between the profile of extraction after tannic acidtreatment with or without glutaraldehyde. Omitting tannic acid in the fixation results in an almost complete extraction of DMPC during the first processing steps.

\subsection{DISCUSSION}

The present findings clearly indicate that tannic acid prevents the extraction of phosphatidylcholine, but in addition also induces the formation of multilamellar aggregates, when added to a suspension of small unilamellar vesicles. Tannic acid initially induces aggregation of the unilamellar vesicles, possibly by interacting with the choline head-groups of the lipids in the bilayers. The induced aggregation is immediately followed by fusion or collapse, leading to tightly packed lamellar structures. The interaction of tannic acid with the choline head-group may influence the electrostatic or hydrative repulsive forces between lipid bilayers and, hence, facilitate aggregation and fusion of the wesicles. Altematively tannic acid may modulate the phase behaviour of phosphatidy/choline since membrane fusion often occurs in bilayers a few degrees below a phase-shift (67).

The aggregation. as induced by tannic acid treatment of phosphatidylcholine 
TABLE 2.1, PRESERVATION OF ${ }^{14} \mathrm{C}$-DMPC DURNNG PROCESSING

Processing step

FIXATHON

Fixation + rinse

Osmiun + rinse

UA in acetone

DEHYDRATION

Acetone-70

Acetone-90

Acetone-100

Acetone + Epon

Epon

TOTAL EXTRACTED

TOTAL RETAINED IN PELLET

TOTAL RADIOACTIVITY
INFILTRATION

Method of fration (\% of radioactivityextracted ${ }^{\mathrm{a}}$ ) fixation GATA fixation TA fixation GA

$\begin{array}{lll}0.58 \pm 0.22 & 0.55 \pm 0.05 & 98.60 \pm 0.14 \\ 1.53 \pm 0.01 & 2.11 \pm 0.48 & 0.45 \pm 0.07 \\ 0.66 \pm 0.08 & 0.81 \pm 0.04 & 0.50 \pm 0.01 \\ 0.20 \pm 0.03 & 0.53 \pm 0.08 & - \\ 1.01 \pm 0.64 & 1.02 \pm 0.63 & - \\ 0.81 \pm 0.46 & 1.04 \pm 0.88 & - \\ 4.83 \pm 0.09 & 3.22 \pm 0.31 \\ 0.48 \pm 0.20 & 0.63 \pm 0.08 & - \\ 9.85 \pm 0.77 & 10.01 \pm 0.99 & 100 \\ 90.11 \pm 0.81 & 90.06 \pm 0.93 & 0 \\ & & \\ 2.11 \pm 0.03 & 2.10 \pm 0.04 & 2.38 \pm 0.05\end{array}$

Values are means $\#$ standard deviation $(n=2)$. ${ }^{b}$ dpnn $\times 10^{6}$;

UA refers 10 uranyl acetate, GA to gludatdeliyde and TA 10 tannic acid.

vesicles. is independent of the composition of the acylchains, thength or degree of unsaturation, but cloes have infuence on the periodicity of the multilamellar structures. The tannic acid concentration has no influence on the periodicity but correlates. with the number and size of the vesicles formed. Post-fixation with osmium letroxide is necessary to prevent complete extraction of phospholipids even after treatment with tannic acid, indicating that the rannic acid-phospholipid complex is atabilized by osmication.

The corollary of these "in vitro" findings is that the results obtained in biological material should be interpreted with care. For instance. in ischemic and reperfused rabbit myocardium multilamellar extrusions adjacent 10 milochondria and sarcolemma have been observed after fixation with glutaraldehyde and tarnic acind (164. 189. 193). The present findings in the phospholipid model system suggest that these multilamellar vesicles do not necessarily represent the "in vivo" situation. II cannot be excluded that lamellar vesicles arc induced by the treatment with lannic 
acid. Pilot studies in our lab indicate that the action of tannic acid on the ischemic myocardium can be explained as an increased availability of phospholipids (notably PC) in the membranes for the interaction with tannic acid. Tannic acid treatment can therefore be used to probe alterations in membrane phospholipids. In this context it is noteworthy that changes in membrane contents and composition have been reported for anoxic cultured cardiac myocytes. This was demonstrated by a change in the lateral diffusion of a lipid probe (74) as well as by a change in the PE/PC labelling ratio upon exposing the living cells 10 an iodinating medium (246).

\subsection{SUMMARY}

The use of tannic acid has been proposed to improve the preservation of phospholipids in tissues. We investigated the effects of tannic acid on the preservation of small unilamellar vesicies, prepared from sonicated aqueous suspensions of phospholi pids.

With cryo-electron microscopy it is demonstrated that small unilamellar vesicles are formed after sonication of the phospholipid suspensions. Fixation of vesicles without tannic acid results in extraction of the phospholipids during dehydration and embedding. Fixation of vesicles containing phophatidylcholine with tannic acid, with or without glutaraldehyde, results in a fast (within a second) aggregation of the vesicles and the resulting sediment can be dehydrated and embedded when a post-fixation in osmium tetroxide is carried out. Small unilamellar vesicles treated in this way are retrieved in thin sections as multilamellar vesicles with a periodicity of about $5 \mathrm{~nm}$ for dimyristoylphosphatidylcholine and about $6 \mathrm{~nm}$ for dioleylphosphatidylcholine.

By using ${ }^{\mid 4}$ C-phosphatidylcholine it was also demonstrated that tannic acid prevents to a large extent the extraction of phosphatidylcholine during fixation. dehydration and embedding. This dual effect, aggregation and fixation. of tannic acid on phosphatidylcholine should be considered when using tannic acid in tissue preparation. 


\section{CHAPTER 3}

THE FORMATION OF MULTILAMELLAR VESICLES BY THE ADDITION OF TANNIC ACID TO PHOSPHATIDYLCHOLINE CONTAINING SMALL UNILAMELLAR VESICLES

Based on: A.H.G.J. Schrijvers, P.M. Frederik, M.C.A. Stuart, K.J.N. Burger, V.V.Th. Heijnen, G.J. Van der Vusse, R.S. Reneman (1989) J Histochem Cytochem (in press). 


\subsection{INTRODUCTION}

In several studies tannic acid has been used to improve the ultrastructural preservation of lipid structures in tissues. Inclusion of tannic acid in the fixative has been employed to improve the preservation of the lamellar-bodies in type II pneumocytes of lung tissue $(12,124)$. Tannic acid has also been applied to retain multilamellar, lipidic extrusions in isolated ischemic and reperfused rabbit hearts (164) as well as for the preservation of lipid droplets in the adrenal cortex of the rat (154).

It is known that lipids (triglycerides, phospholipids and others) are especially susceptible to extraction during tissue processing (224). Although tanmic acid is not required for the preservation of arachidonoyl phospholipids (134), it has been proposed as an additive to the fixative to improve the preservation of lipid structures in tissue preparation, particularly lipid structures containing a high proportion of saturated phosphaticlylcholines. This can be explained by assuming that tannic acid interacts with the choline headgroup of phosphatidylcholines and that the resulting complex is stabilized by post-fixation with osmium tetroxide ( $(25)$.

In our laboratory we have demonstrated that tannic acid reduces the extraction
of ${ }^{14} \mathrm{C}$-phosphatidylcholine from small unilamellar vesicles (194). Tannic acid also induces aggregation and the formation of multilamellar structures when added to small unilamellar vesicles containing either dimyristoylphosphatidylcholine (DMPC) or dioleoylphosphatidylcholine (DOPC) $(191,194)$. Since lipid structures in tissue are often found to have a lamellar appearance, the question arises whether this represents their appearance in the living-state or whether this lamellar appearance is induced by the treatment with tannic acid.

The present study was designed to investigate in more detail the initial events in the aggregation of small unilamellar vesicles, as induced by tannic acid. Studies of aggregation and formation of multilamellar vesicles were performed using synthetic phospholipids. It was felt that experiments with biological phospholipids were necessary to facilitate the interpretation of these findings to effects of tannic acid on tissue preparation, to this end phospholipids extracted from tissues, either purified (commercially obtained) or as total phospholipid fraction extracted from rabbit or rat hearts were used as well. A model system was used with phospholipid suspensions, 
sonicated to form small unilamellar vesicles.

Turbidity measurements were performed to examine the kinetics of the tannic acid reaction with sonicated vesicles. Cryo-microscopic techniques (freeze-fracturing and cryo-electron microscopy of thin films) were used to investigate the ultrastructure of wesicles during the initial reaction with tannic acid.

In addition we have used conventional thin-section electron microscopy, which in contrast to the previously mentioned techniques is used in routine tissue preparation. In this way it could be examined whether similar observations can be made with the different techniques. Several primary fixation protocols were used to examine whether the multilamellar vesicles formed were of the same type for the different phospholipids. It was also investigated whether the induction of aggregation and the formation of multilamellar vesicles, as caused by tannic acid, had a specificity for phosphatidylcholine (PC).

\subsection{MATERIAL AND METHODS}

3.2.1. The preparation of small unilamellar vesicles.

Small unilamellar vesicles were prepared by sonication of phospholipid suspensions. Initially the phospholipids were dissolved in a chloroform/methanol mixture (9/1) and then were dried under a gentle stream of dry $\mathrm{N}_{2}$-gas. Subsequently the lipid residues were suspended in a concentration of $5 \mathrm{mg}$ phospholipid//ml phosphate (20 $\mathrm{mM}$ ) buffered physiological saline (PBS. pH 7.2) and sonicated for 10 second periods at 10 second intervals until a clear to slightly opalescent suspension was obtained. The phospholipids used were either dimyristoylphosphatidylcholine (DMPC; P0888. Sigma St.Louis, synthetic), dioleoylphosphatidylcholine (DOPC; Sigma P1013, synthetic), Eggyolk phosphatidylcholine (Egg-PC: Sigma P5763), a combination of phosphatidylserine (PS: Sigma P8518, extracted from bovine brain) and DMPC (2/3 molar ratio), a combination of PS and phosphatidylethanolamine (PE; Sigma P9137, extracted from bovine brain) ( $9 / 1$ molar ratio) or a total lipid extract from rabbit or rat hearts. 
TABLE 3.1. Total contem and relative fatty acid composition of the various phospholipid subelasses extracted from nornoxic rabbil heart.

\begin{tabular}{|c|c|c|c|c|c|c|c|c|}
\hline \multirow[t]{2}{*}{ FMTTY ACID } & \multicolumn{8}{|c|}{ LIPID SUBCLASSES relative composition (\%) } \\
\hline & $\mathrm{LPC}$ & LPE & SPH & PS & Pl & $\mathrm{PE}$ & $\mathrm{PC}$ & $\mathrm{DPG}$ \\
\hline 14:0 & - & 0.8 & 0.1 & 0.1 & 0.3 & 0.2 & 0.1 & 0.8 \\
\hline $1.4: 0 d$ & - & . & - & .. & . & . & 0.1 & - \\
\hline $16: 0$ & 25.0 & 6.1 & 19.4 & 1.4 & 4.1 & 23.6 & 3.8 & 2.6 \\
\hline $16: 0 d$ & . & - & - & - & - & 4.7 & 8.6 & - \\
\hline $16: 1$ & - & . & - & - & 0.4 & 0.2 & 0.1 & 0.6 \\
\hline $18: 0$ & 33.3 & 45.3 & 19.6 & 52.4 & 44.2 & 7.0 & 24.6 & 2.0 \\
\hline $18: 00$ & - & - & - & - & - & 2.2 & 8.8 & - \\
\hline $18: 1$ & 16.3 & 11.3 & 1.1 & 9.9 & 7.8 & 16.9 & 5.6 & 6.0 \\
\hline 18:1d & - & . & - & - & - & 2.0 & 3.6 & - \\
\hline $18: 2$ & 15.5 & 18.5 & 1.1 & 12.5 & 15.5 & 22.2 & 10.5 & 85.2 \\
\hline $18: 3$ & - & - & - & - & - & 0.4 & 0.2 & 1.2 \\
\hline $20: 0$ & - & - & 22.5 & 1.6 & 0.2 & 0.1 & 0.1 & 0.2 \\
\hline $20: 1$ & - & - & - & 0.6 & .. & 0.1 & 0.1 & - \\
\hline $20: 4$ & 5.2 & 18.3 & 0.5 & 11.2 & 27.0 & 10.5 & 31.8 & 1.5 \\
\hline $22: 0$ & . & $=$ & 24.9 & 1.5 & m & 0.1 & - & - \\
\hline $22: 4$ & . & . & - & 4.8 & 0.6 & 0.4 & 0.8 & 0.4 \\
\hline $22: 6$ & . & - & - & 3.8 & 0.1 & 0.4 & 1.4 & - \\
\hline 240 & - & - & 6.1 & 0.3 & - & - & - & - \\
\hline $24: 1$ & - & - & 4.8 & - & . & . & - & . \\
\hline TOTAL CONTENT & 0.4 & 0.6 & 5.1 & 5.4 & 10.1 & 80.6 & 118.1 & 38.9 \\
\hline
\end{tabular}

Total content is expressed as unoll fatty acid moieties per gram of dry weight of tissue. LPC, LPE, SPH, PS, PI, PE, PC and DPG refer to lyso-phosphatidylcholine, lyso-phosphatidyl. erhanolamine, sphingomyelin, phosplatidylserine, phosphatidylinositol, phosphatidylethanolamine, phosphatidylcholine and diphosphatidyglycerol (cardiolipin), respectively. Fatty acid moieties are denoted according to their chemical notation. 14:0d, 16:0d, 18:0d and 18:td refer to hemiacetals formed by action of methyl groups on the ether linkage of the aliphanic chain to the glycerol backbone (plasmalogen form of the phospholipid).

3.2.2. Exiraction and analysis of the phospholipids from hearts.

Hears of two 10 weeks old Lewis rats (mildly anesthetized with diethylether) and of a 2 months old New Zealand white rabbit (stunned by a blow in the neck. causing cervical dislocation) were removed after thoracotomy and mounted on a perfusion apparatus. The hearts were perfused for several minutes, with carboxygenated $\left(95 \% \mathrm{O}_{2}\right.$ and $5 \% \mathrm{CO}_{2}: \mathrm{pO}_{2}>80 \mathrm{kPa}$ ) Tyrode buffer to remove the blood. The ventricles of the hearts were freeze-clamped with aluminum tongues pre-cooled in 
TABLE 3.2. Total content and relative fatry acid composition of the various phospholipid subclasses extracted from normoxic rat hearts.

\begin{tabular}{|c|c|c|c|c|c|c|c|c|}
\hline \multirow[t]{2}{*}{ FATTY ACID } & \multicolumn{8}{|c|}{ LIPID SUBCLASSES relative composition (\%) } \\
\hline & LPC & LPE & SPH & $\mathbb{P S}$ & PI & $\mathrm{PE}$ & $\mathrm{PC}$ & DPG \\
\hline $14: 0$ & - & 7.2 & 0.9 & . & $\overline{0.6}$ & - & - & 0.6 \\
\hline $14: 0 \mathrm{~d}$ & - & - & - & - & - & - & - & - \\
\hline $16: 0$ & 26.2 & - & 14.2 & 1.8 & 4.0 & 8.9 & 17.2 & 2.7 \\
\hline $1600 \mathrm{~d}$ & - & - & - & - & - & 6.5 & 1.5 & - \\
\hline $16: 1$ & - & - & - & - & - & 0.1 & 0.2 & 0.3 \\
\hline $18: 0$ & 58.5 & 46.4 & 31.8 & 53.2 & 44.8 & 24.8 & 28.5 & 2.8 \\
\hline $18: 0 \mathrm{~d}$ & - & - & - & - & - & 4.9 & 0.2 & - \\
\hline $18: 1$ & - & 7.2 & 0.9 & 5.0 & 4.8 & 4.6 & 7.8 & 5.2 \\
\hline $18: 1 \mathrm{~d}$ & - & * & - & - & - & 2.5 & 0.2 & - \\
\hline $18: 2$ & 10.8 & - & 1.8 & 4.1 & 8.0 & 7.8 & 17.2 & 83.0 \\
\hline $18: 3$ & - & - & - & - & - & 0.2 & 0.2 & 0.4 \\
\hline $20: 0$ & - & - & 16.0 & - & - & - & 0.1 & - \\
\hline $20: 1$ & - & - & - & . & - & 0.1 & 0.1 & - \\
\hline $20: 4$ & 4.6 & 19.6 & 2.7 & 6.7 & 33.4 & 16.7 & 21.2 & 2.0 \\
\hline $22: 0$ & - & - & 14.2 & - & - & $=$ & 0.1 & 0.1 \\
\hline $22: 4$ & - & - & $\cdots$ & 1.8 & - & 0.4 & 0.2 & - \\
\hline $22: 6$ & - & 19.6 & 1.8 & 27.4 & 4.2 & 22.6 & 5.3 & 2.8 \\
\hline $24: 0$ & - & - & 8.8 & . & - & - & - & - \\
\hline $24: 1$ & - & - & 7.0 & - & - & - & - & - \\
\hline TOTAL CONTENT & 0.6 & 0.4 & 3.6 & 7.1 & 11.2 & 98.5 & 113.6 & 43.8 \\
\hline
\end{tabular}

Total content is expressed as $\mu$ mol fatty acid moieties per gram of dry weight of tissue. See for further explanation the legend of Table 3.1.

liquid nitrogen. Then the frozen tissues were pulverized in an aluminum mortar with a stainless steel pestle, both pre-cooled in liquid nitrogen. The tissue powder was transferred to a test tube and the lipids were extracted by addition of a mixture of chloroform/methanol (2/1 by volume), as described before (237).

To delineate the proportion of the various phospholipid subclasses in the lipid mixture. phospholipids were separated from neutral lipids by silicagel column chromatography (174). Subsequently the phospholipids were separated into their subclasses with the use of thin-layer chromatography, as earlier described (234). The fatty acid moieties in the various phospholipid subclasses were determined by gas-liquid chromatography (237). The lipid content is expressed in gram per dry weight. To this end pre-weighed aliquots of the frozen tissue were freeze-dried overnight to assess the dry weight of the tissue aliquots. 


\subsubsection{Turbidity measurements.}

To study the effects of tannic acid concentration on the induction of phospholipid aggregation turbidity measurements were performed with sonicated vesicles containing either DMPC (1 mM) or DOPC $(0.5 \mathrm{mM})$ to which tannic acid (Mallinckrodt Inc AR 1764) was added in (final) concentrations of $0.005 \%$ to $0.04 \%$ (w/v). The change in optical density. at $405 \mathrm{~mm}$, was recorded on a spectrophotometer, at $37^{\circ} \mathrm{C}$, one minute before and during 10 minutes after tannic acid was added to the vesicle preparation.

Since the precise composition of the tannic acid used, referred to by the manufacturer as chiefly digallic acid $\left(\mathrm{C}_{14} \mathrm{H}_{10} \mathrm{O}_{9}\right)$, is not known we assumed a molecular weight of 322 for the tannic acid employed.

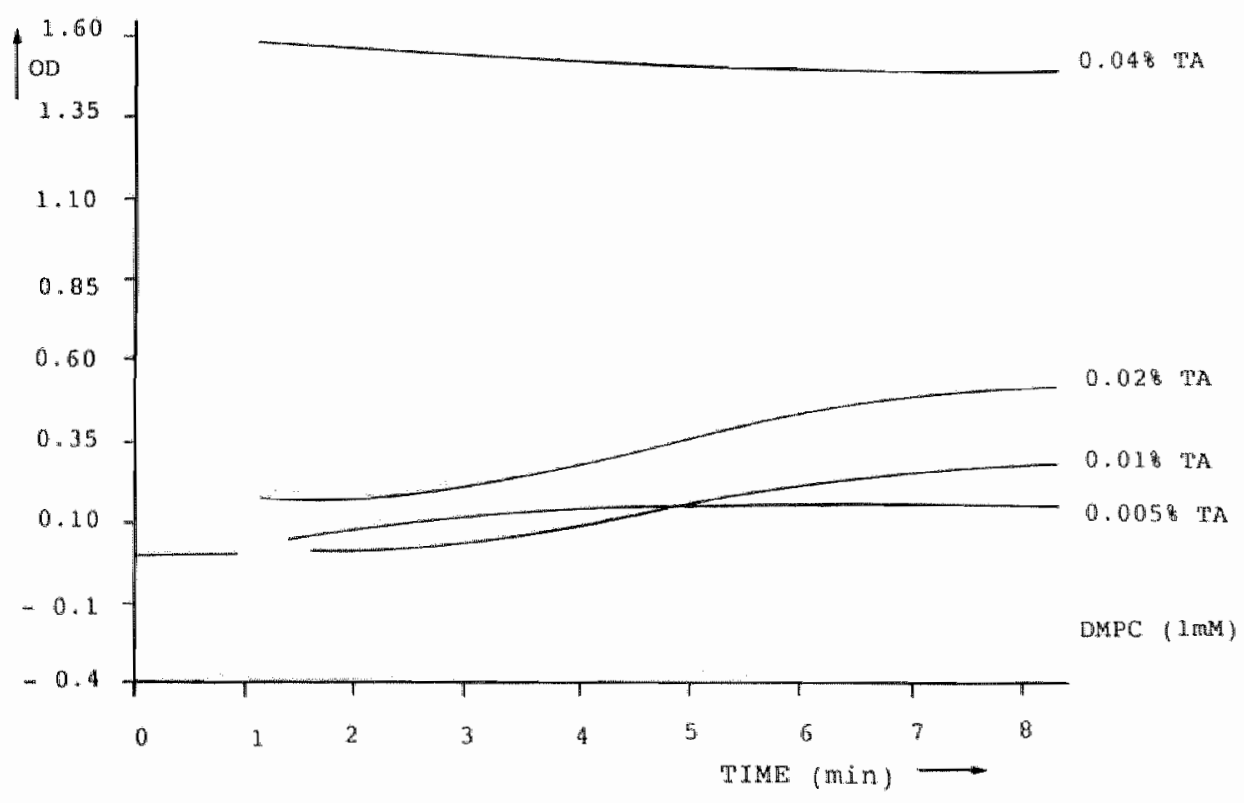




\subsubsection{Cryo-electron microscopy of thin films.}

Sonicated vesicle preparations were observed "directly" by cryo-electron microscopy of thin films $(85,86)$. A bare coppergrid (hexagonal 700 mesh; without supporting film) was dipped in a sonicated phospholipid suspension, containing small unilamellar vesicles. The grid was then blotted with filter paper to remove the excess of fluid and a thin film formed between the bars of the grid. A thin film formed in this way consists of a phospholipid monolayer at each air-water interface and the enclosed aqueous phase containing small unilamellar vesicles $(85,86)$. Subsequently the grid was vitrified in liquid ethane (64), transferred to the electron microscope (Philips $\mathrm{CM} 12,100 \mathrm{KeV})$ and observed at $-190^{\circ} \mathrm{C}$ (139). Deposition of vitrified water on the specimen is prevented by shielding the specimen during transfer to the microscope column and during the initial and transient rise of the partial vapour pressure of water in the high vacuum of the microscope column (86). The micrographs obtained have only little contrast, due to the fact that thin-film preparations with phospholipid vesicles in focus are devoid of contrast $(65,86)$. Micrographs were therefore taken 3$6 \mu \mathrm{m}$ under focus to improve (phase-)contrast.

To study the initial aggregation of sonicated vesicles, as induced by tannic acid, we also vitrified thin films ( $5 \mathrm{mg} \mathrm{DMPC} / \mathrm{ml} \mathrm{PBS}$ ) within a few seconds after the addition of tannic acid (AR 1764, Mallinckrodt Inc.) at final concentrations of $0.04 \%$, $0.1 \%$ and $0.2 \%$ in PBS. These concentrations are lower than those used in the conventional transmission electron microscopic experiments. This was done because at higher concentrations of tannic acid the aggregation of the phospholipid vesicles was too fast (within 1 second) for the preparation of thin films.

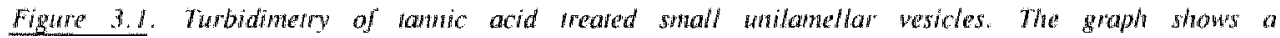

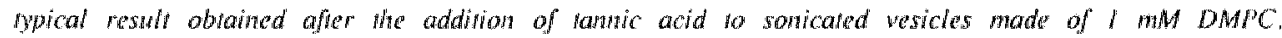

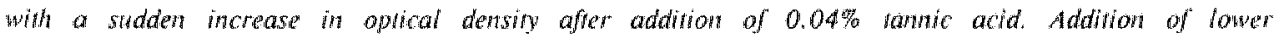

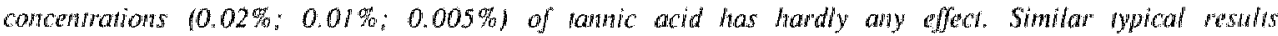
are obrainch with sonicared vesicles made of DOPC. 


\subsubsection{Freeze-fracture electron microscopy.}

In order to substantiate the results obtained by cryo-electron microscopy of thin films fast-freezing was combined with freeze-fracturing. Small aliquots $(2 \mu 1)$ of a sonicated vesicle preparation made of DMPC $(10 \mathrm{mg} / \mathrm{ml})$ were mixed in equal volume with a tannic acid solution (both in $20 \mathrm{mM}$ PBS. pH 7.2). The final concentration of tannic acid was varied from $0.005 \%$ to $0.3 \%$ (w/v). The samples were fast-frozen within 30 to 40 seconds after mixing (at room temperature) in a thin copper sandwich by plunging them into liquid propane $\left(-190^{\circ} \mathrm{C}\right.$ ), using a plunge freezing device (KF80; Reichent Jung). No cryo protectants were used. Freeze-fracture and replication were performed (at $-105^{\circ} \mathrm{C}$ ) following established procedures, using a BAF400 (Balzers) freeze-etch machine. The replicas were stripped off and cleaned (overnight) with commercial bleach and distilled water, and examined (at $80 \mathrm{KeV}$ using a Philips EM420.

\subsubsection{Conventional thin-section electron microscopy.}

Several treatments were carried out to inwestigate the effects of tannic acid on the preservation of phospholipids and the ultrastructure of small unilamellar vesicles when using glutaraldehyde fixation. A primary fixation was performed at room temperature for 10 minutes, using different solutions with a respective final concenIration of:

a) PBS (20 $\mathrm{mM}$ phosphate buffered physiological saline, pH 7.2) (no primary fixation. velnicle only).

b) $0.5 \%$ glutaraldehyde (A2523, Biorad) in PBS (20 mM, pH 7.2),

c) $0.4 \%$ tannic acid (AR 1764. Mallinckrodt Inc.) in PBS (20 mM, pH 7.2),

(l) $0.5 \%$ glutaraldehyde and $0.4 \%$ tannic acid in PBS $(20 \mathrm{mM}, \mathrm{pH} 7.2)$.

e) $2.5 \%$ glutaraldeltyde and $2 \%$ mannic acid in PBS $(20 \mathrm{mM}, \mathrm{pH} 7.2)$, which is equal to a fixation protocol often used in tissue preparation.

Each primary fixation was followed by post-fixation with $1 \%$ osmium tetroxide in PBS (20 mM. pH 7.2), for \& hour at room temperature. Subsequently the preparations were stained en-bloc with $1 \%$ uranyl acetate in $50 \%$ acetone. dehydrated with acerone 

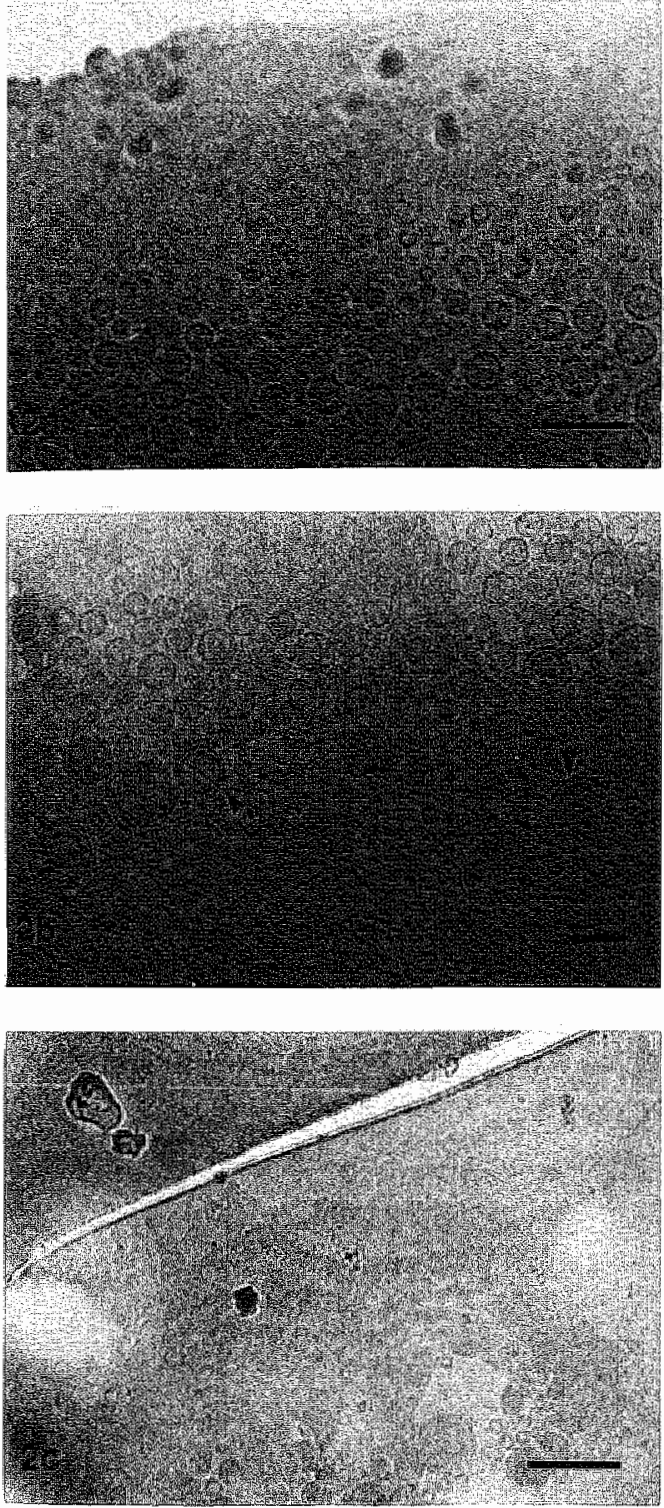

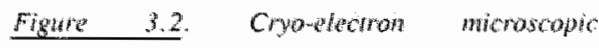
observarions of thin jilms.

(a) Sinall minlamethor vesiches, whith a diomeler of about 40 an made by sonication of a DMPC saspension are "dincty" absented by

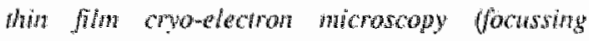

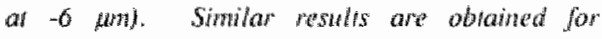
all oher sonicared phospholipid suspensions. The baw represens 0.1 wh.

(b) Micrograph (fochssing at -3 um) of a small unilameltar vesicle mode of $D M P C$. virnjied shorly affer the addirion of $0.04 \%$ rambic acid (fmal concemration). Notice that some vesicles have one of more contact silfes (arrowheats). The bar represens 0.1 Am.

(c) In other preparations of tonnric arid (0.1\%) ireaned DMPC-vesictes a fiesion of vesicles can be sean leading ro sherer fomation of phosphatipids focussing at -3 am). The bar represents 0.5 um. 
and embedded in Epon 812. Thin sections were contrasted with uranyl and lead stains and examined in the electron microscope using a Philips EM400 at $100 \mathrm{KeV}$. The periodicity of the tannic acid induced multilamellar vesicles was measured and expressed as the center to center distance between two adjacent dark lines (lamellar spacing). This was established for several multilamellar vesicles containing either DMPC, DOPC or Egg-PC (Table 3.4).

\subsection{RESULTS}

\subsubsection{Phospholipids extracted from heart tissue.}

The content of the various phospholipid subciasses and their relative fatty acid composition in rabbit and rat hearts are shown in Table 3.1 and 3.2 , respectively. The content of the phospholipid subclasses did not substantially differ between the two species. The proportion of plasmalogen, measured as dimethylacetal in the assay rechnique used, was found to be high in the rabbit PC and low in the rat PC rabout $19 \%$ and $1.5 \%$, respectively). The plasmalogen part of the PE subclass showed a less striking difference (11.8\% and $9.9 \%$ for rabbit and rat hearts, respectively). A moderate difference was also observed in the proportion of sphingomyelin $(1.97 \%$ and $1.27 \%$ for rabbit and rat hearts, respectively), being approximately $36 \%$ lesser in rat hearts. A striking feature was that the fatty acid composition of sphingomyelin was almost exclusively composed of saturated fatly acids. "Tannic acid can therefore be considered the only compound possibly interacting with sphingomyelin during fixation, since both glutaraldehyde and osmium tetroxide are not capable of reacting with saturated sphingonyelins. Furthemore it should be noted that the faty acid composition of both the plasmalogen counterpart of phosphatidylcholine and of lyso-phosphatidylcholine was also almost exclusively composed of saturated fatty acids. With respect to the relative fatty acid composition of the various subclasses it should be noted that the presence of docosahexaenoic acid (C 22:6) was more marked in rat than in rabbit heart phospholipids. Paimitic acid (C 16:0) was more abundantly present in rat PC than in rabbil $\mathrm{PC}$. 

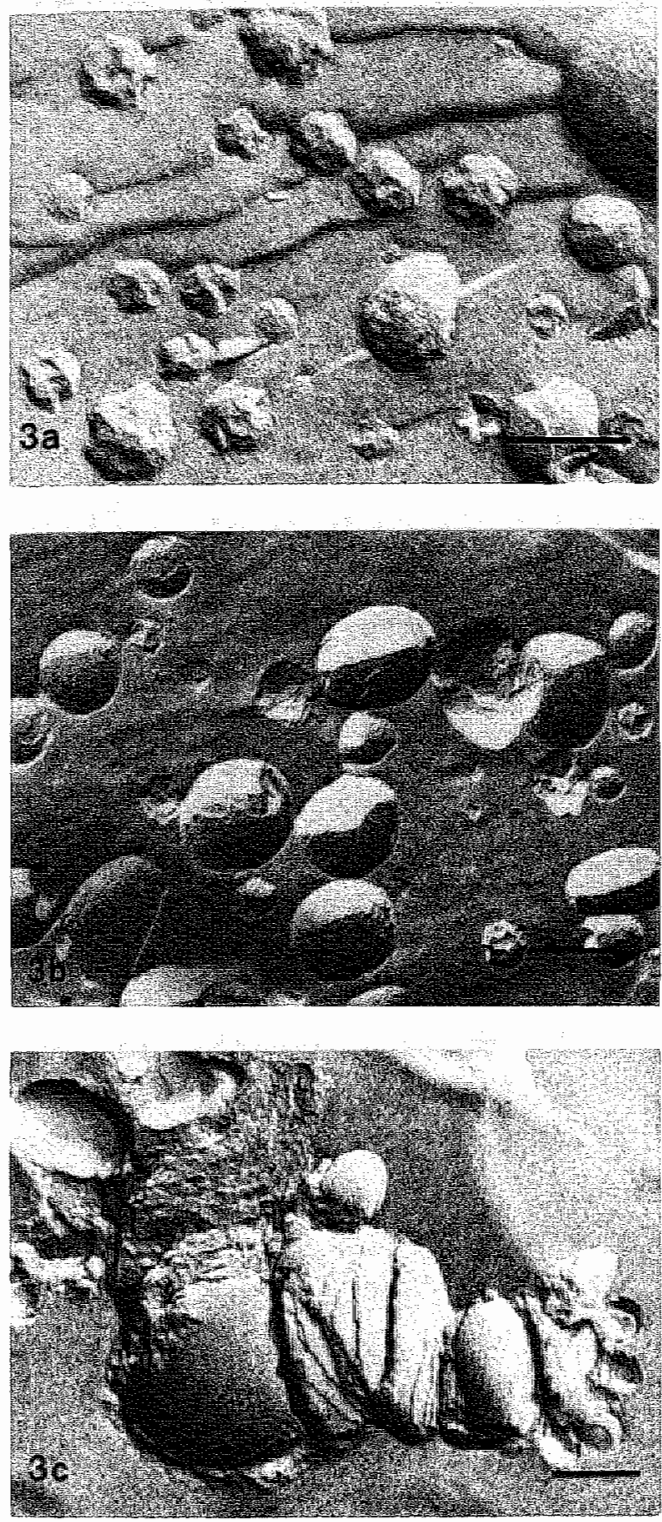

Figure 3.3. Freew-fracture electron micoscopy of sonicated wesicles made of $D M P C$.

(a) Aggregaion and clusicrs of vesicles art obserwed in a sonicated westole prepharation 15 mg DMPC/ $m$ ). fast frozen shortiy after the addition of $0.05 \%$ tannito acid. The bar repressivits $0.2 \mu \mathrm{m}$.

(b) The chasters apparently fiese or collapse to form relatively snall mulitannellas vesticies $10.05 \%$ sanmic acid). The bat represents 0.2 pum.

(c) Addition of higher concentmations of rannic acid $(0.3 \%)$ to the unilantellar vesiches leads to the jormation of large mulvilamellar structures. The bar represents $0.2 \mu \mathrm{m}$. 

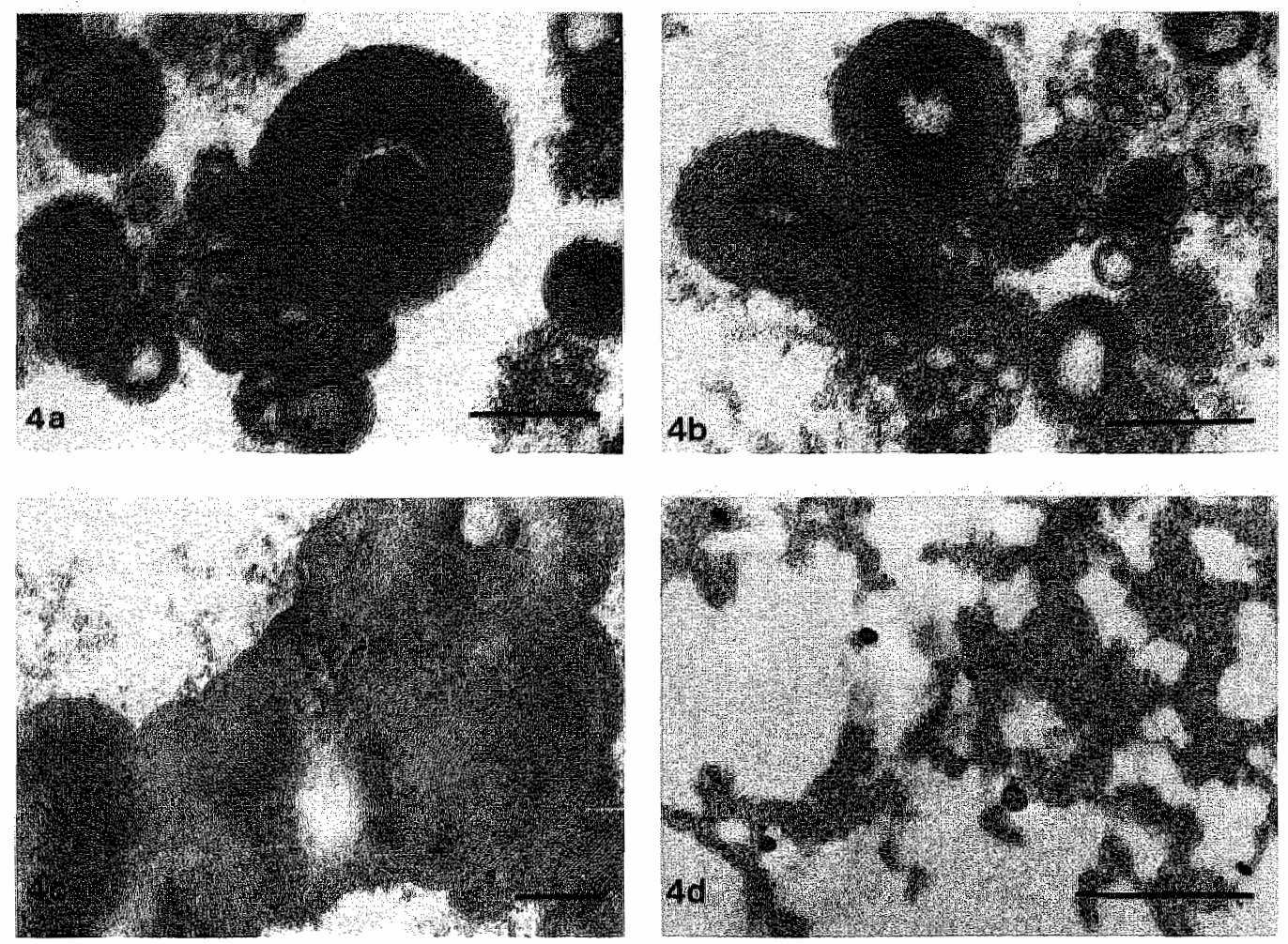

Figue 3.4. Thin-section electron microscopic observations of symthenic phosphouph vestcles.

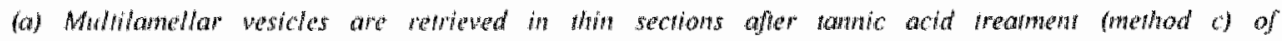

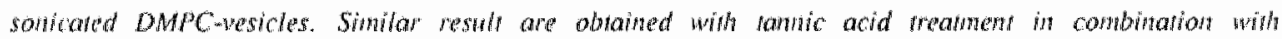

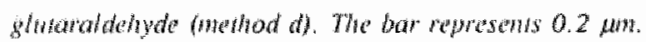

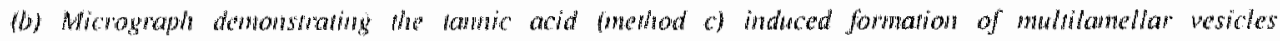

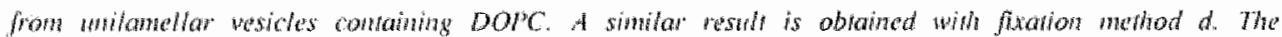
bar represenss 0.2 wint

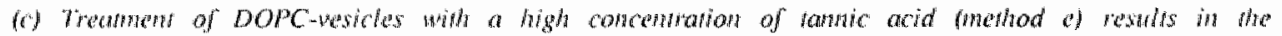

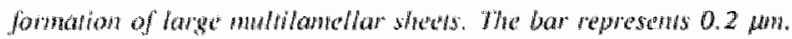

(d) Wicrosmoph showing antorhous smonkes as found in win sections affer glutaraldehyde fixation

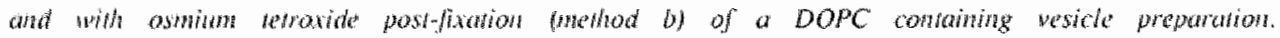

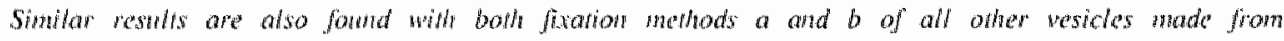

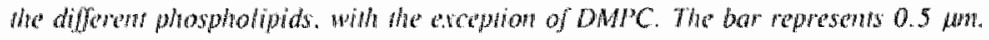




\subsubsection{Turbidity measurements.}

The influence of tannic acid concentrations on the induction of aggregation was examined by measuring the change in optical density of a vesicle preparation, made from DMPC, upon the addition of tannic acid. A threshold concentration of tannic acid $(0.04 \%)$ was found necessary for rapid and massive aggregation of the phospho. lipid vesicles (Fig. 3.1). A similar typical result was obtained with a vesicle preparation made from DOPC (not shown).

\subsubsection{Thin film cryo-electron microscopy.}

Small unilamellar vesicles, formed by somication, were visualized by cryo-electron microscopy of thin films. In Figure 3.2a an example is given of a vesicle preparation containing DMPC. Similar results were obtained for all other vesicle preparations of the different phospholipids or phospholipid mixtures investigated (not shown).

Using this technique it could be demonstrated that the addition of tannic acid llo small unilamellar vesicles, macle of DMPC. caused fusion and aggregation possibly inducing the formation of multilamellar vesicles. The vesicles showed several sites of contact, where fusion events or collapse could occur (Fig. 3.2b). The formation of phospholipid sheets from the vesicles could also be observed (Fig. 3.2c).

\subsubsection{Freeze-fracture electron microscopy.}

Freeze-fracture electron microscopy of untreated vesicle preparations revealed small. unaggregated vesicles of approximately $40-50 \mathrm{~nm}$ (not shown). It was further" demonstrated that in the initial phase of the reaction of tannic acid with DMPC. sonicated vesicles aggregate to form small clusters of a defined size $(100-200 \mathrm{~nm}$, Fig. 3.3a). During the course of the tannic acid treatment these clusters are readily destabilized and collapse to form multilamellar structures (Fig. 3.3b and $3.3 \mathrm{c}$ ). Clusters of (unilamellar) vesicles could onlly be seen at low concentrations of tannic acid $(\leqslant 0.1 \%)$, at high concentrations of tannic acid $(\geqslant 0.3 \%)$ the reaction was so fast that 
upon freezing within approximate 30 seconds after the addition of tannic acid only large multilanellar structures were found (Fig. 3.3c), resembling those found in thinsections after plastic embedding (see below).

\subsubsection{Conventional thin-section electron microscopy.}

When tannic acid, either with or without glutaraldehyde was added (methods c,d and e) to sonicated vesicles containing PC a very fast (within I second) aggregation took place. It should be emphasized that this phenomenon was found only for the vesicle preparations containing PC and thus not found for the PS/PE-preparation.

All the phospholipid aggregates, induced by tannic acid, could be pelleted and further processed for embedding. However, it was necessary to perform a post-fixation with asmium tetroxide before organic solvents could be applied for the dehydration. Deletion of the post-fixation resulted in dissolving of the phospholipid aggregates during the final dehydration steps and therefore no material was retrieved in thin sections.

Similar multilamellar vesicles were retrieved in thin sections after tannic acid treatment, with or without glutaraldehyde (methods $c, d$ and e), for all the different sonicated vesicle preparations containing PC (Table 3.3). Examples of the effect of tannic acid on small unilamellar vesicles made of DMPC or DOPC are shown in Figure 3.4. In thin sections of the Egg-PC preparation not only multilamellar vesicles, accounting for approximately $70 \%$ of the observed structures, were found but also (approximately $5 \cdot 10 \%$ ) large multilamellar sheets and (20-30\%) lamellar structures strongly resembling the lamellar-bodies found in type II pneumocytes of lung tissue (Fig. 3.5). The large lamellar-body like structures showed some similarity with the coexisting multilamellar sheets and might have developed from these structures by partial extraction of phospholipids during processing. Tannic acid also induced aggregation of vesicles prepared from the lipids extracts from rabbit or rat hearts (Fig. 3.6). However, a striking quantitative difference was observed; substantially more multilamellar vesicles were found in the rabbit heart preparation than in the rat heart preparation (an approximate two-fold difference in the number of vesicles was assessed per imagelield in the electron microscope at the same magnification). 
Formation of multilamellar vesicles
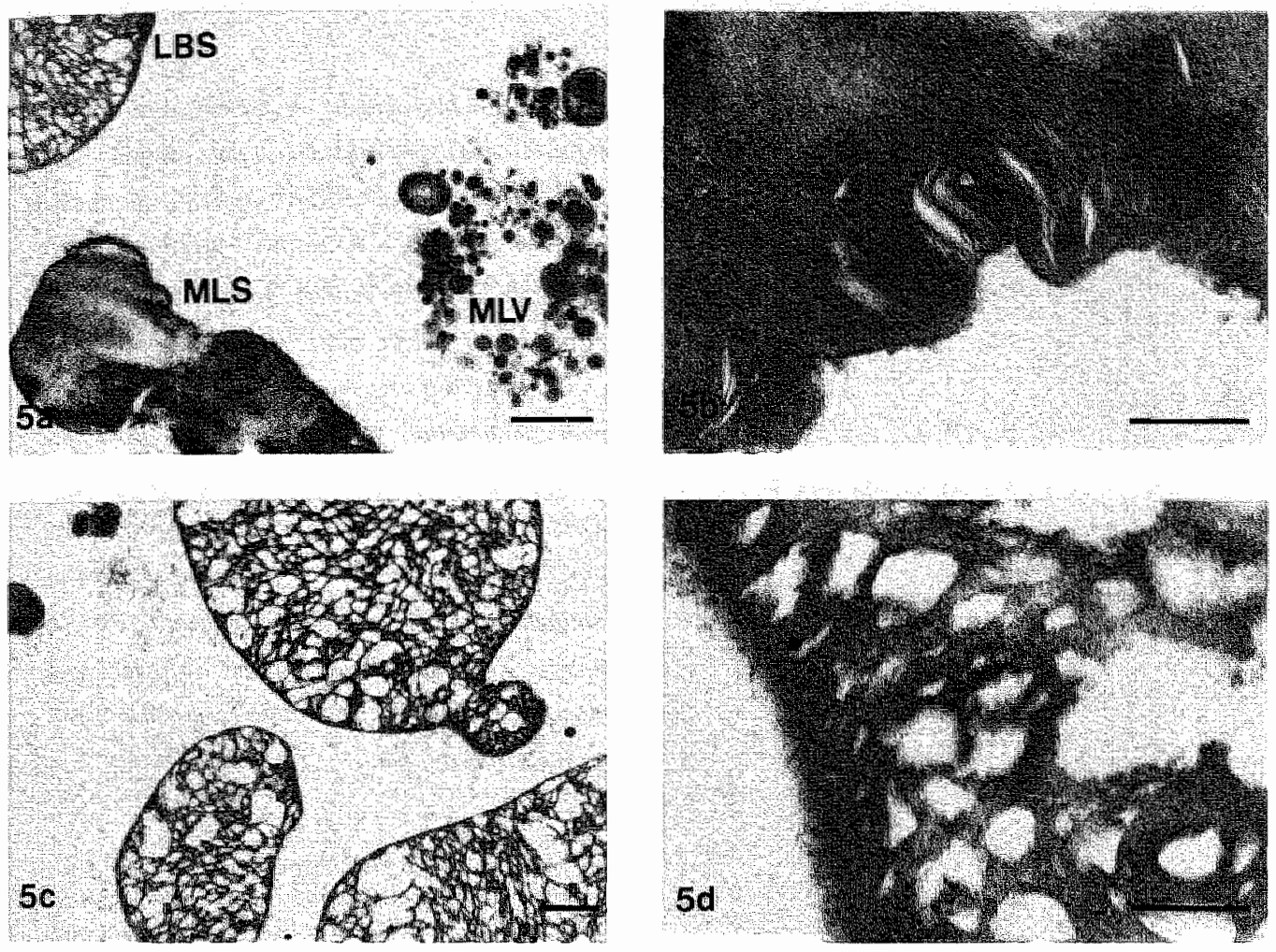

Figure 3.5. Thin-section elecrom microscopic obserwations of unilanellar vesicles made of Egs-PC and trealed winh tamic acid.

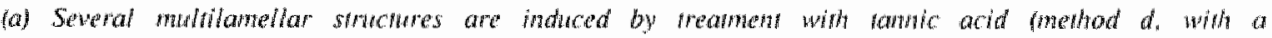

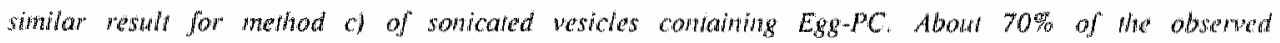

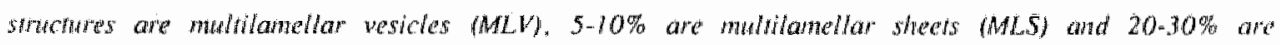
lamellar-body like struchures (LBSI. The bar represents / HWh.

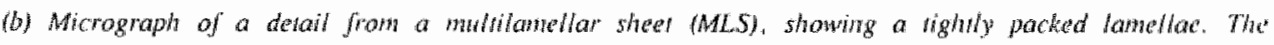
bar represents 0.2 wm.

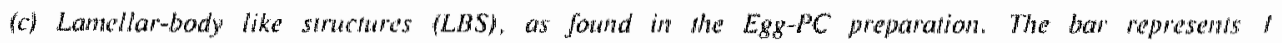
HW.

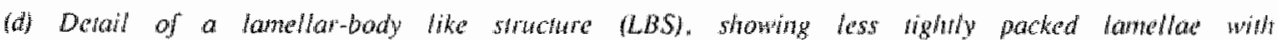
woriable interlawellar distances. The bar represenrs 0.2 fun. 

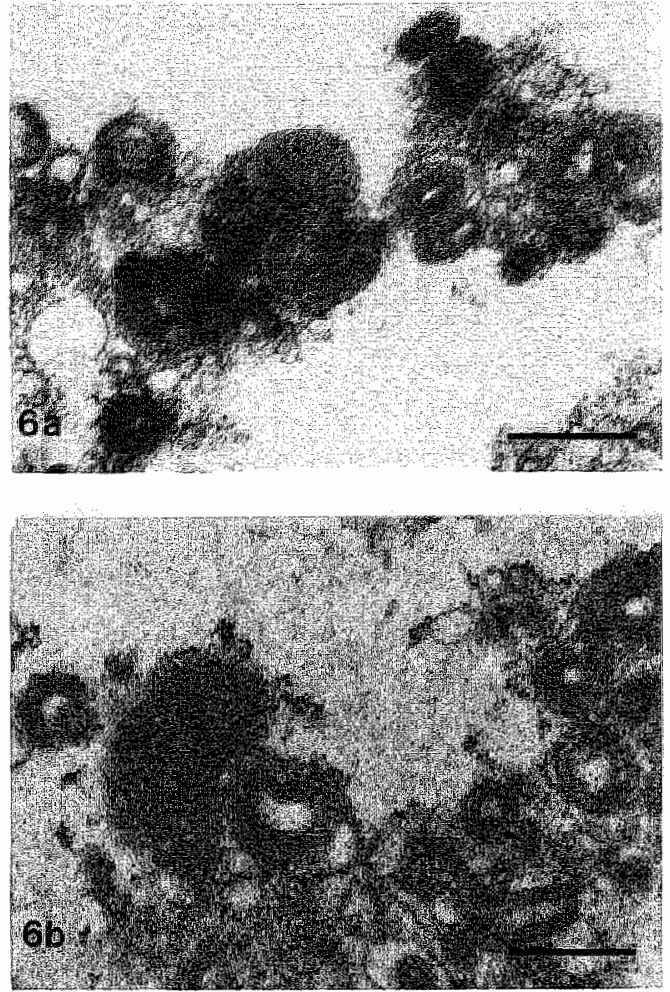

Fighne 3.6. Mhorographs of tannic acid meared vestales, made of the low lipit extracts from rabibil or rat hearts penreved in thinsechions.

(a) Mulihamellar vesicles abserved th thit sections afler tannic acid reament fromod $c$. a simblar resuh is found with menthod d) of stwall unilatellar wesicles prepared from the lipid extract front the rabbil heart. The bar represents 0.2 Hath.

(b) Tannic acid fomenthod cl induced fornation of mwhilamellow vesicles frow sorticaned vesicles made of the lipid extract from rat hearts, as observed in win sections after embedding. The bar represents 0.2 AMH.

Multilamellar vesicles, similar to those shown in Figure 3.6, were also found in thin sections after tannic acid fixation (methods c,d) of sonicated vesicles containing PS/DMPC.

Treatment of the small unilamellar vesicles with a high tannic acid concentration $(2 \%)$ in combination with (2.5\%) glutaraldehyde (method e) resulted in the formation of large multilamellar sheets in addition to multilamellar vesicles. An example of a vesicle preparation containing DOPC. treated in this way is shown in Figure 3.4.

Fixation of small unilamellar vesicles with glutaraldehyde and post-fixation with osmium tetroxicle or post-fixation with osmium tetroxide without primary fixation (methods a and b, thus without the use of tannic acid) did not lead to proper preservation of the unilamellat vesicles. In thin sections of the different vesicle preparations used only amorphous structures could be found (see Table 3.3). An example of sonicated DOPC-vesicles, fixed with osmium tetroxide alone (method a), is 
shown in Figure 3.4 .

There was no significant difference in the periodicity (lamellar spacing) found for each of the examined vesicle preparations treated with tannic acid with or without glutaraldehyde (methods $c$,d) (Table 3.4). The periodicity of the vesicles was significant larger for DOPC than for DMPC (P<0.001) or Egg-PC $(\mathbb{P}<0.005)$, whereas no difference in periodicity was found between the vesicles made of $D M P C$ on $\mathrm{Egg}-\mathrm{PC}$ (two sample T-test).

TABLE 3.3. Structures retrieved in thin sections of embedded small unilamellar vesicles prepared from different phospholipids and treated with several fixation methods.

\begin{tabular}{|c|c|c|c|c|c|c|c|}
\hline \multirow[t]{2}{*}{ TREATMENT } & \multicolumn{7}{|c|}{ VESICLES PREPARED FROM : } \\
\hline & DMPC & DOPC & Egg-PC & RBL & RTL & PS/DMPC & $\mathrm{PS} / \mathrm{PE}$ \\
\hline . & m & $\mathrm{A}$ & A & $\overline{\text { M.d. }}$ & $\overline{\text { n.d. }}$ & A & $\mathrm{A}$ \\
\hline $0.5 \% \mathrm{GA}$ & - & A & A & A & $A$ & A & A \\
\hline $0.4 \% \mathrm{TA}$ & $\mathrm{M}$ & M & M & $\mathrm{M}$ & $M$ & $M$ & - \\
\hline $0.5 \% \mathrm{GA}+0.4 \% \mathrm{TA}$ & M & $\mathrm{M}$ & L. & M & M & M & - \\
\hline $2.5 \% \mathrm{GA}+2 \% \mathrm{TA}$ & $s$ & $S$ & n.d. & n.d. & nid. & ildi. & A \\
\hline
\end{tabular}

- None, $A=$ Amorphous structures, $M=$ Mullilamellar vesicles, $S=$ multilanellar Sheets, $L=m$ Lamellar-body like structures with multilamellar vesicles and multilamellar shees, n.d. = not determined. $\mathrm{GA}=$ glutaraldelyyde and $\mathrm{TA}=$ tannic acid. DMPC, DOPC, Egg-PC, RBL, RTL PS, PE refer to dimyristoylphosphatidylcholine, dioleoylphosphatidylcholine, Egg-yolk phosplkatidylclooline. rabbit lipid exiract, rat lipid extract, phosphatidylserine and phosphatidylethanolamine, respectively. PS/DMPC and PS/PE are mixed in a $2: 3$ and $9: 1$ molar ratio, respectively.

\subsection{DISCUSSION}

Tannic acid was first introduced in electron microscopy by Mizuhira and Futaesaku in 1971 (145). Tannic acid is a natural product with a complex (and variable) composition. Its reaction mechanisms are still incompletely understood. Its effects during tissue preparation vary depending on the stage at which tannic acid is introduced into the preparation protocol and are therefore altematively referred to as "fixation" (124, 145), "staining" (229) or "mordanting" (205, 252). In the latter case tannic acid was considered as a mordant between osmicated structures in the tissue and lead stains. The structures or substructures that are affected by the mordant do 
also depend on the stage at which tannic acid is introduced into the preparation protocol $(178,188,189,205,252)$. Thus, tannic acid can be used to increase and diversify the contrast in tissues by its mordanting effect. However, the effects of tannic acid as described in this paper are likely to be of different nature. since tannic acid is applied before osmication.

Tannic acid is also known to interact with the choline headgroup of phosphatidylcholine (125). In this context it is often concluded that tannic acid prevents extraction of phosphatidylcholine and therefore the use of tannic acid is often advocated to improve the ultrastructural preservation of lipid structures.

From the data presented in this paper it can also be concluded that tannic acid is beneficial in reducing the extraction of phosphatidylcholines, since the lipid vesicles survived dehydration and embedding after tannic acid based fixation. This conclusion is supported by the findings in previous experiments in which the extraction of ${ }^{14} \mathrm{C}$ DMPC from DMPC containing vesicles was measured during the processing for electron microscopy (194). It was found that $90 \%$ of the radioactivity is retained in the embedled pellets after fixation with tannic acid, with or without glutaraldehyde. whereas the radioactivity was lost during the first processing steps when performing glutaraldehyde fixation without tannic acid. The findings in the present study also demonstrate that tannic acid induces aggregation of small unilamellar vesicles specifically containing phosphatidylcholine and the formation of tightly packed multilamellar structures. We assume that the action of tannic acid is independent of glutaraldehyde, since the formation of multilamellar vesicles is similar in the treatments with and without glutaraldehyde (methods $c$ and d). Besides, the periodicity of the multilanellar vesicles does not differ irrespective of the fixation method used. The extraction of phosphatidy/choline during processing of small unilamellar vesicles, containing ${ }^{14} \mathrm{C}-\mathrm{DMPC}$. is the same for both fixation methods ( $c$ and d) (194).

The findings with cryo-electron microscopy of thin films indicate that tannic acid induces aggregation of vesicles and sheet formation. The induced aggregation leads to the formation of clusters of vesicles, probably followed by fusion or collapse. and the formation of multilamellar structures, as observed with freeze-fracturing. This is most likely caused by interaction of tannic acid with the choline headgroups of the phospholipids in an approximate one-to-one molar ratio, as established in the turbidity 
experiments. This might be concluded since fast and massive aggregation of sonicated vesicles, containing DMPC $(1 \mathrm{mM})$, occurred after addition of $0.04 \%$ tannic acid, which equals $1.2 \mathrm{mM}$ tannic acid.

Regarding the mechanism(s) underlying these findings one can only speculate upon. One possibility is that the interaction of tannic acid with the choline headgroups of the phospholipids in the bilayer arrangements influences the electrostatic or hydration repulsive forces between the lipid bilayers and, hence, facilitates aggregation and/or fusion of the vesicles. How this subsequently may result in tightly packed multilamellar structures is still unknown.

One of the most striking findings using conventional transmission electron microscopy was that all the tannic acid treated unilamellar vesicles, specifically containing PC, were retrieved in thin sections as multilamellar structures, sometimes strongly resembling lipid structures found in tissue, using tannic acid during processing $(19,63,124,164,178,189.251)$. It is a matter of debate as to whether the lamellar appearance of the lipid structures, as often found in tissues, is a correct reflection of the appearance in the living state, considering the fact that tannic acid has such different effects and that the underlying reaction mechanisms are still incompletely understood. It has been suggested that the appearance of lipid structures in tissue, as for example the lamellar-bodies, in the electron microscope depends on the way of preparation $(15,98,112,135,226)$. Our present "in vitro" findings support this notion because the lamellar appearance of the lipidic structures might well be induced by the treatment with tannic acid. This is in agreement with 1) the appear= ance of lamellar globules in the caulonema tip cells of the moss Funaria hygrometrica. after prolonged fixation containing tannic acid (251) and 2) the conclusion of Saffitz and co-workers (178) that the development of multilamellar extrusions from the cell membrane of myeloma cells was due to treatment with tannic acid (before osmication).

In ischemic and reperfused isolated rabbit myocardium multilamellar vesicles have been observed after fixation with a combination of glutaraldehyde (2.5\%) and tannic acid $(2 \%)$. Multilamellar vesicles were found in myocytes, extruding from mitochondria and sarcolemma (164), adjacent to lipid droplets and extruding from endothelial membranes $(189,193)$. The present findings, regarding the unilamellar vesicles. strongly suggest that the lamellar structures, as found in the ischemic and/or 
reperfused hearts, are induced by tannic acid. Although multilamellar extrusions have been observed in ischemic myocardium. without the use of tannic acid (164). the multilamellar vesicles do not necessarily represent their "in vivo" appearance when tannic acid based fixation is used. We tend to explain the action of tannic acid in the ischemic myocardium as reflection of an increased availability of phospholipids in the membranes to an interaction with tannic acid. It is likely that this changed interaction specifically refers to phosphatidylcholines and probably the plasmalogen phosphatidalcholine and sphingomyelin. This is suggested by the observed difference in the reaction of tannic acid with small unilamellar vesicles made of the lipid extracts from rabbit or rat hearts. which is most likely explained by their differences in the proportion of the plasmalogen of phosphatidylcholine and possibly the differential content of sphingomyelin.

Further investigations are required to rate the true value of using tannic acid induced multilamellar vesicles as a parameter to estimate the ischemia and/or reperfusion induced damage in cardiac myocytes.

TABLE 3.4. Periodicity of lamellae in multilamellat wesicles retrieved in thin sections after tanmic acid treatment. Mean values and standard deviations are presented.

\begin{tabular}{lllll}
\hline PHOSPHOLIPID & FIXATION & PERIODICITY & MEASUREMENTS \\
\cline { 2 - 4 } DMPC & GATA & & $4.9 \pm 0.5 \mathrm{~nm}$ & $\mathrm{n}=16$ \\
DOPC & GATA & $6.4 \pm 0.5 \mathrm{~nm}$ & $\mathrm{n}=14$ \\
Egg-PC & GA/TA & $5.2 \pm 0.7 \mathrm{~nm}$ & $\mathrm{n}=5$ \\
& & & \\
DMPC & TA & $4.6 \pm 0.3 \mathrm{~nm}$ & $\mathrm{n}=8$ \\
DOPC & TA & $6.2 \pm 0.4 \mathrm{~nm}$ & $n=5$ \\
Jgg-PC & TA & $4.8 \pm 0.7 \mathrm{~nm}$ & $\mathrm{n}=7$ \\
\hline
\end{tabular}

The periodicity in DOPC tontaining multilamellar vesicles was significant larger than for the preparations containing DMPC $(\mathrm{P}<0.001)$ or Egg $\mathrm{PC}(\mathrm{P}<0.005)$ (two sample $\mathrm{T}$-test), itrespective of the applied fixation $(0.4 \%$ tanic acid (TA) or $0.5 \%$ glutaraldehyde and $0.4 \%$ tannic acid (GATA), methods $\mathrm{c}$ and a, respectively. DMPC, DOPC. Egg-PC refer to dimyristoylphosphatidylcholine, dioleoylphosphatidylcholine and Egg-yolk phosphatidylcholine, respectively. 


\subsection{SUMMARY}

Tannic acid induces aggregation and the formation of multilamellar vesicles when added to preparations of small unilamellar vesicles specifically containing phospharidylcholine. Aggregation and clustering of vesicles was demonstrated by cryo-electron microscopy of thin films and freeze-fracture technique. Turbidity measurements revealed an approximately one-to-one molar ratio between tannic acid and phosphatidylcholine necessary for a fast and massive aggregation of the small unilamellar vesicles. When the tannic acid induced aggregates were dehydrated and embedded for conventional thin-section electron microscopy multilamellar vesicles were retrieved in thin sections. It is concluded from morphological studies as well as tracer studies that tannic acid, at least to a large extent, prevents the extraction of phosphatidy/choline. Multilamellar vesicles were also observed in tannic acid treated vesicles prepared from the total lipid extracts from either rabbit or rat hearts. Substantially more multilamellar vesicles were retrieved in the rabbit vesicle preparation. This difference can probably be explained by the difference in the proportion of the plasmalogen of phosphatidylcholine, and the differential content of sphingomyelin. in the lipid extracts of rabbit and rat hearts. It is concluded that the dual effect (reduced extraction and aggregation) of tannic acid on phosphatidylcholines should be taken into consideration when using tannic acid in tissue preparation. 



\title{
CHAPTER 4
}

\begin{abstract}
INFLUENCE OF THE DURATION OF ISCHEMIA AND REPERFUSION ON MULTILAMELLAR VESICLES IN ISOLATED RABBIT HEARTS: AN ULTRASTRUCTURAL AND MORPHOMETRICAL STUDY USING TANNIC ACID BASED FIXATION
\end{abstract}

Based on: A.H.G.J. Schrijwers, M.J.M. de Groot, V.V.Th Heijnen, G.J. Van der Vusse. P.M. Frederik, R.S. Reneman (1989) J Mol Cell Cardiol (prowisionally acepted for publication). 


\subsection{INTRODUCTION}

One of the characteristic changes occurring during myocardial ischemia is an increase in the permeability of the sarcolemma, as manifested by the release of macromolecules such as cellular enzymes (105). The mechanisms responsible for ischemic cell injury, leading to sarcolemmal dysfunction, are not fully elucidated yet $(121,127,242)$. Several morphological changes of the sarcolemma have been observed in anoxic or ischemic myocytes, such as pealing of the glycocalyx (80), aggregation of intramembranous particles $(7,83,164)$, formation of microblebs (161) or subsarcolemmal blebs (179), and rupture of the sarcolemma $(80,119,221)$. Extrusions of multilamellar, liposome-like structures from mitochondria and sarcolemma have been observed "with the use of tannic acid based fixation $(165,189,193)$.

Little is known about 1) the nature of these multilamellar vesicles. 2) the time scale of development (in relation to the duration of ischemia). 3) the effects of reperfusion, 4) the number and localization of the multilamellar vesicles (cellular and subcellular distribution), 5) their size and possible differences in morphological architecture, and 6) the formation of multilamellar vesicles in relation to other ischemia induced morphological changes. Further insight into these features is necessary to understand the pathophysiological importance of the observations made and to rate the true value of the multilamellar vesicles as a parameter to quantitate ischemic (membrane) injury.

Therefore, a more detailed study was designed to investigate the influence of the duration of ischemia and reperfusion on the formation of multilamellar vesicles. For this purpose isolated rabbit hearts were made ischemic for various intervals, a subset of which was reperfused for 30 minutes after the ischemic period. Hearts were fixed by perfusion with a combination of glutaraldehycle $(2.5 \%)$ and tannic acid $(2 \%)$ and further processed for electron microscopic examination. The study was focused on the number and size of the multilamellar vesicles, found in thin sections, and on their (sub-)cellular location. Moreover. it was investigated as to whether differences in the morphological architecture (size and periodicity of lamellae in multilamellar vesicles) could be observed between multilamellar extrusions at different locations or under the different experimental conditions. In addition the relation between the number of 
multilamellar vesicles and the severity of the myocardial ischemic injury, as scored on a semi-quantitative scale, was studied to gain more insight into the usefulness of multilamellar vesicles as an indicator of ischemic (membrane) injury.

\subsection{MATERIAL AND METHODS}

\subsubsection{Preparation of the hearts.}

The experiments were performed on male New Zealand White rabbits, weighing between 2.5 and $3 \mathrm{~kg}$ (approximately 3 months old). After heparinization (1000 IE $/ \mathrm{kg}$ body weight, i.v.) the rabbits were stunned by a blow in the neck causing cervical dislocation. Subsequently the hearts were rapidly removed by thoracotomy and immediately immersed in ice-chilled Tyrode's solution. The hearts were weighed after remnant lung tissue and fat had been removed. The hearts were then attached to the aortic cannula of the perfusion apparatus, installed in a thermostated housing, in which the environmental temperature was held constant at $37^{\circ} \mathrm{C}$, as described before (210).

\subsubsection{Perfusion conditions.}

The hearts were retrogradely perfused, according to Langendorff (136), with a modified Tyrode's solution [containing; $130 \mathrm{mM} \mathrm{NaCl}$, 5.6 mM KCl, $2.2 \mathrm{mM} \mathrm{CaCl}_{2}, 1.0$ $\mathrm{mM} \mathrm{MgCl}, 1.2 \mathrm{mM} \mathrm{NaH}_{2} \mathrm{PO}_{4}$, and $28.6 \mathrm{mM} \mathrm{NaHCO}_{3}$, with $\mathrm{D}(+)$ Glucose $(11.0 \mathrm{mM})$ and Pyruvate $(5.0 \mathrm{mM}$, sodium salt) added as substrates]. The perfusion medium was continuously oxygenated (with $95 \% \mathrm{O}_{2}$ and $5 \% \mathrm{CO}_{2} ; \mathrm{pO}_{2}>80 \mathrm{kPa}$ ) and fillered $(1.2 \mu \mathrm{m}$ Milipore filter) during recirculation. The temperature of the medium was kept constant at $37^{\circ} \mathrm{C}$, whereas the $\mathrm{pH}$ varied between 7.35 and 7.45 . A catheter connected to a pressure transducer (CTC) was inserted into the left ventricle through the apex to measure left ventricular pressure. 


\subsubsection{Experimental conditions.}

After the connection to the perfusion apparatus was completed the hearts were perfused retrogradely $(L)$ during a 30 or 150 minutes (control) period at a constant pressure of $8.0 \mathrm{kPa}\left(30^{\circ} \mathrm{L}, \mathrm{n}=5\right.$ and $150^{\circ} \mathrm{L}, \mathrm{n}=3$, respectively).

In another set of experiments the hearts were subjected to 30 . 60 or 90 minutes of global, normothermic $\left(37^{\circ} \mathrm{C}\right.$ ). nomflow ischemia (I) after an initial 30 minutes retrograde perfusion $\left(30^{\circ} \mathrm{L}+30^{\prime} \mathrm{I}, \mathrm{n}=4 ; 30^{\circ} \mathrm{L}+60^{\circ} \mathrm{l}, \mathrm{n}=3\right.$ and $30^{\prime} \mathrm{L}+90^{\prime} \mathrm{I}, \mathrm{n}=4$, respectively). Ischemia was accomplished by complete occlusion of the cannula connected with the left atrium and the aorta.

In a third set of experiments the ischemic periods were followed by a retrograde reperfusion of 30 minutes (R), under the same conditions as during the initial control period $\left(30^{\circ} \mathrm{L}+30^{\prime} \|+30^{\circ} \mathrm{R}, \pi=3 ; 30^{\prime} \mathrm{L}+60^{\prime} \mathrm{I}+30^{\circ} \mathrm{R}, \mathrm{n}=3\right.$ and $30^{\circ} \mathrm{L}+90^{\circ} \mathrm{I}+30^{\circ} \mathrm{R}, \mathrm{n}=5$, respectively).

The temperature of the hearts was monitored with thermoprobes throughout the experiments and was kept at $37 \pm 1{ }^{\circ} \mathrm{C}$.

\subsubsection{Fixation and tissue processing.}

At the end of the experiments the hearts were fixed by perfusion (at $8 \mathrm{kPa}$ ) with a combination of $2.5 \%$ glutaraldehyde (Biorad: A2523) and $2 \%$ tannic acid (Mallinckrodt Inc; AR 1764) in $20 \mathrm{mM}$ phosphale buffered physiological saline (PBS. pH 7.2) at $25^{\circ} \mathrm{C}$ during 10 minutes. Midwall biopsies (about $1 \mathrm{~mm}^{3}$ ) were cut from the left ventricle and were further fixed by immersion at room temperature for 2 hours.

After fixation the lissue pieces were rinsed in $0.1 \mathrm{M}$ phosphate buffer $(\mathrm{PB}, \mathrm{pH}$ 7.2), post-fixed in $1 \% \mathrm{OsO}_{4}$ in $0.1 \mathrm{MPB}(\mathrm{pH} 7.2)$ at room temperature for 1 hour and rinsed twice in $0 . \llbracket \mathrm{MPB}(\mathrm{pH} 7.2)$ and $50 \%$ acetone. Subsequently samples were stained en bloc with $1 \%$ Uranyl Acetate in $50 \%$ acelone at $4^{\circ} \mathrm{C}$ for 7 to 10 hours, dehydrated in acetone and embedded in Epon 812. Thin sections were contrasted according to routine procedures and examined in the electron microscope (Philips EM400 at 100 KeV). 


\subsubsection{Morphometric analysis.}

For each experiment 10 to 15 micrographs were taken randomly from one grid at the same magnification. These micrographs were analyzed morphometrically (Kontron; MOP-videoplan) to establish the number, size and (sub)cellular location of multilamellar vesicles. To this end the following parametters have been regarded: the respective areas of the cardio-myocytes, nuclei, mitochondria, lipid droples and multilamellar vesicles examined on the micrographs.

Furthermore, the length of the sarcolemma was determined on the micrographs, and the number of mitochondria, lipid droplets and multilamellar vesicles. Results are expressed per experimental group as mean values and standard errors of the mean, and statistically analyzed for differences between the groups using the Mann-Whitney $U$ test. Differences in the size of the multilamellar vesicles were evaluated for statistical significance using the (unpaired) Student T-test. P-values less than 0.05 were considered to be statistically significant.

The sum of multilamellar vesicles extruded from the sarcolemma, mitochondria, and lipid droplets and of the multilamellar vesicles that could not be clearly classified as originating from any of these structures was considered to be (MLV-TOTAL) and is expressed as $\mathrm{n} / \mathrm{mm}^{2}$ of myocardial tissue examined. The mean number of multilamellar vesicles extruded from the sarcolemma (MLV-SL) was corrected for a possible nonrandom sampling error by expressing the number of MLV-SL per mm sarcolemma. This was achieved by dividing the sum of the observed MLV-SL in each heart preparation by the corresponding length of the sarcolemma examined. A similar correction was made for multilamellar vesicles extruded from mitochondria (MLV-MITO) and adjoining lipid droplets (MLV-LIPID) by expressing the number of multilamellar vesicles per mitochondrion or per lipid droplet, respectively (Fig. 4.3). Since the number of multilamellar vesicles adjacent to lipid droplets appeared not to be related to the ischemic insult, the number of observed multilamellar vesicles (MLV-TOTAL) was also determined without taking the number of multilamellar vesicles adjacent to or inside lipid droplets (MLV-LIPID) into consideration (Fig. 4.2b). In addition, we estimated the amount of phospholipids extruded from the sarcolemma. To this end, the tolal lengih of membrane-lamellae in multilamellar vesicles was estimated and divided by the length 
of sarcolemma examined, assuming similar phospholipid densities in the intact and affected sarcolemma (Table 4.3).

The degree of ischemic injury was evaluated on electron micrographs according to a slightly modified semi quantitative scoring system, as described by schaper et al. (185). In short, evaluation of the ultrastructural appearance of mitochondria, nuclei and myofillaments, and the presence or absence of glycogen and edema was expressed in a numerical scone ranging from 0 (normal) to 4 (irreversibly injured). Each tissue sample was scored according to this evaluation system. Data are presented per group as mean scores and standard errors (Fig. 4.2c). The strength of the association between ischemic injury scoring and the amount of multilamellar extrusions was determined using Spearman's rank correlation $\left(\mathrm{R}_{\mathrm{S}}\right)$.

\subsection{RESULTS}

\subsubsection{General morphologic appearance.}

After control perfusion $\left(30^{\circ} \mathrm{L}\right.$ and $150^{\circ} \mathrm{L}$, respectively) typical control tissue with normal mitochondria (sometimes with matrix granules), normal nuclei and normal (slightly contracted) myofilaments were seen. Glycogen was present and neither extracellular nor intracellular edema could be observed

(Fig. 4, 1a).

After a relatively short period of ischemia (30'l) and reperfusion $\left(30^{\prime} \mathrm{l}+\mathrm{R}\right)$ slightly damaged cells were found. The mitochondria had a somewhat cleared matrix. The nuclei had a normal density of chromatin. Several nuclei showed marginal clumping of chromatin. Pants of the observed tissues showed minor extracellular and/or intracellular edema. Glycogen was present in all myocytes.

After 60 minutes of ischemia (60\%) moderately to severely damaged cells were observed. The mitochondria had a cleared matrix and sometimes broken cristae were found. Most nuclei had a light density and showed margination of chromatin. Sometimes extracellular and/or intracellular edema was seen. Limited arnounts of glycogen were observed in some hearts of the ischemic group. In the hearts reper- 
fused following 60 minutes of ischemia $\left(60^{\circ} I+R\right)$ only minor differences were observed, as compared with the corresponding ischemic group. The mitochondrit had more broken cristae. Several nuclei were pyknotic and no glycogen was observed in any of the tissues examined.

After an extended period of ischemia (90'1) severely damaged cells were seen. The mitochondria had a cleared matrix often with flocculent densities and abundantly broken cristae. Most nuclei were pyknotic. The myofilaments were mostly relaxated. Extracellular and/or intracellular edema was present in most hearts, whereas all cells examined lacked glycogen. Reperfused hearts after this period of ischemia $\left(90^{\circ} I+R\right)$ only differed from the ischemic hearts by exhibiting slightly more flocculent densities and more broken cristae in the mitochondria and in one heart by the presence of hypercontraction.

\subsubsection{Ischemic injury scoring.}

The degree of myocardial ischemic injury was found to be drastically increased in hearts that had been ischemic for at least 60 minutes (Fig. 4.2c). Reperfusion after an ischemic period did not lead to an increase in ischemic injury, as basedl upon the scoring method used.

\subsubsection{Multillamellar vesicles.}

Extrusions in the form of concentric multilamellar vesicles were found in endothelial cells (Fig. 4.10) as well as in myocytes. From the latter cell type these vesicles were found extruded from the sarcolemma (Fig. 4. Id), mitochondria (Fig. 4.1e), or adjacent to or inside lipid droplets (Fig. 4.If). The number of multilamellar extrusions varied under the different experimental conditions .

During the shorter periods of ischemia $\left(30^{\circ} 1\right.$ and $\left.60^{\circ} \mathrm{I}\right)$ no change in the mean number of MLV-SL was observed, as compared to the control groups. However, the mean number of MLV-SL increased in hearts reperfused after 60 and 90 minutes of ischemia, the latter increase being significant (Fig. 4.3a).

The mean number of MLV-MITO was small in the control groups $\left(30^{\circ} \mathrm{L}\right.$ and $150^{\circ} \mathrm{L}$ ) 

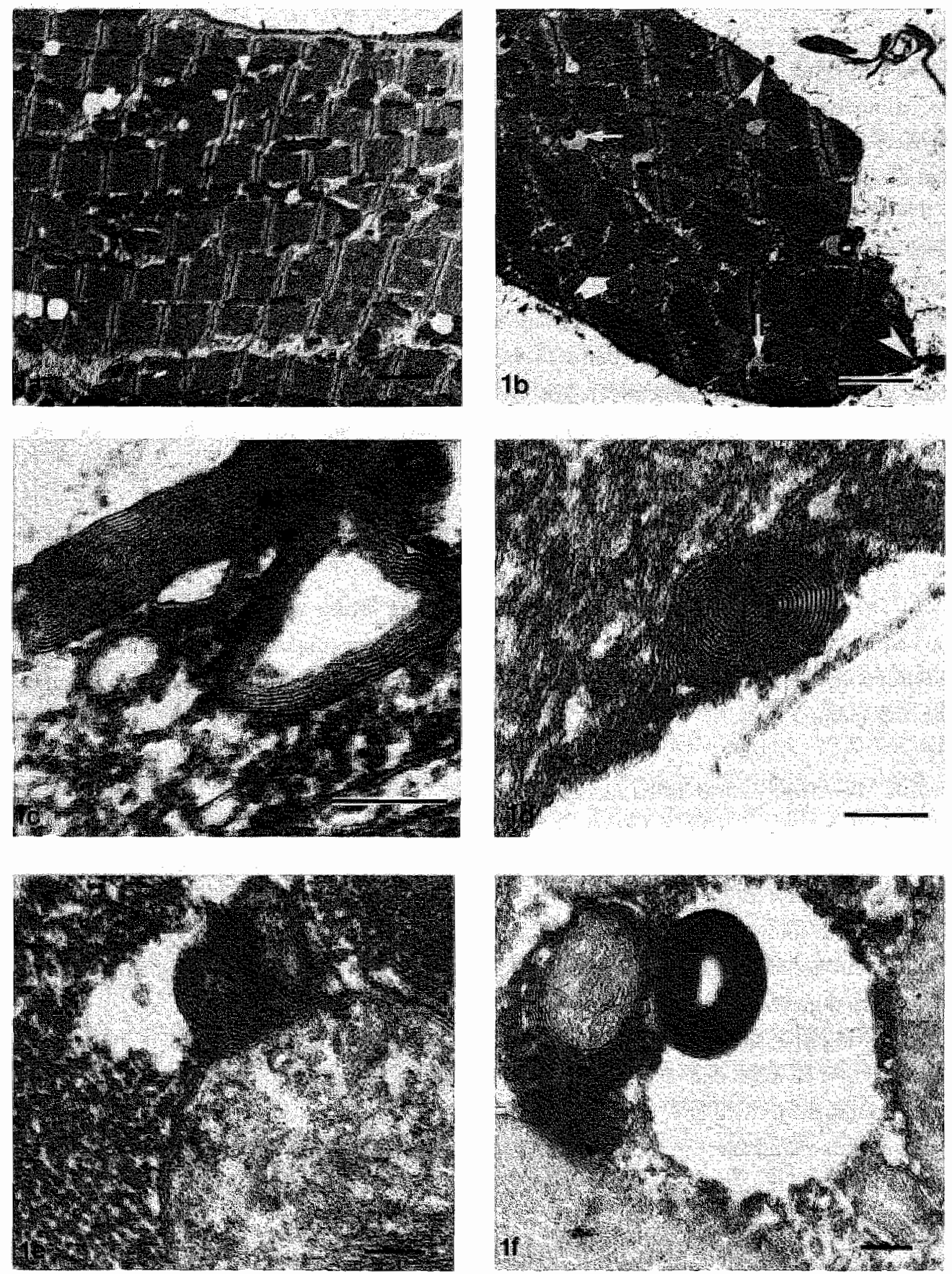
increased after 60 minutes of ischemia and 60 minutes of ischemia followed by reperfusion. The increase in the nean number of MLV-MITO in hearts rendered ischemic for 90 minutes did not reach the level of significance. A significant increase was observed after 90 minutes of ischemia followed by reperfusion (Fig. 4.3b).

The mean number of MLV-LIPID did not change consistently in the groups under" investigation (Fig. 4.3c).

The mean number of MLV-TOTAL was found to be small, but not zero, in hearts normoxically perfused for 30 or 150 minutes (control groups) (Fig. 4.2a). A small increase in the mean number of MLV-TOTAL was found in hearts subjected 10 60 minutes ischemia (Fig. 4.2a). A larger increase in the mean number of MLV-TOTAL was observed after 90 minutes of ischemia and after 60 or 90 minutes of ischemia followed by reperfusion (Fig. 4.2a).

Figure 4.1. Electron tricrographs showing the norphological appearance of control (30L) rissme (a) and of hearts after ischemia and reperfusion $\left(30^{\circ} \mathrm{L}+90^{\circ} \mathrm{l}+30^{\circ} \mathrm{R}\right)(\mathrm{b} \cdot \mathrm{d})$.

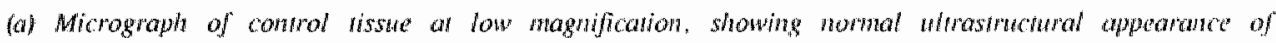
"he cardionyocyes. The ba represents 2 ann.

(b) Micrograph at low magnification, showing the rypical distribution of multilamellat wexiches its

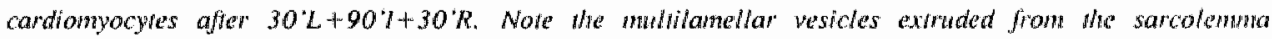

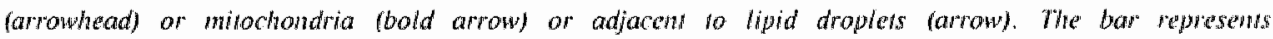
$2 \mathrm{~m}$.

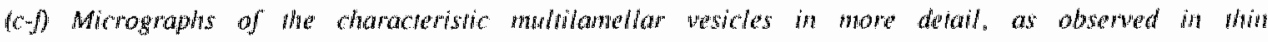
sechions of embedded rabbin mocarditum, fived afe" $30 \mathrm{~L}+90 \%+30 \%$.

16) Typical example of a malibam endothehal cell. The bar represchls 0.2 wh.

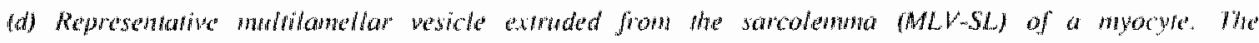
bar represenss 0.1 सh⿻

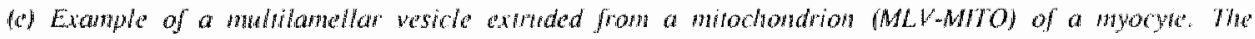
bat represens 0.1 .

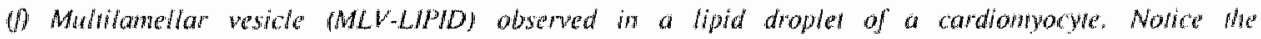

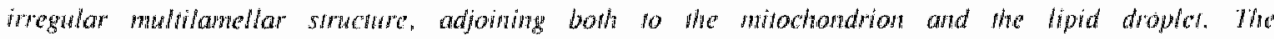
bar represens 0.1 whi. 
When MLVLLIPID was not taken into consideration (see below) in the number of multilamellar vesicles observed (MLV-TOTAL minus MLV-LIPID), the mean number was found to be increased after 60 and 90 minutes of ischemia and even more pronounced when hearts were reperfused (Fig. 4.2b).

In general the relative distribution of multilamellar vesicles in the various ex perimental groups aws as follows: about $25-30 \%$ of the observed multilamellar vesicles were extruded from the sarcolemma (MLV-SL), 25-30\% from mitochondrial membranes (MLV-MITO) and 40-50\% from or inside lipid droplets (MLV-LIPID).

The mean size of MLV-SL, MLV-MITO or MLV-LIPID was not significantly different (data not shown). Although the variation in the size of the MLV-TOTAL was small between the different groups, the mean size of the MLV-TOTAL in the 30 minutes control group was significantly smaller than that in most of the other groups (Table 4,1 and 4.3),

Assuming that the lipid density of the multilamellar vesicles and the sarcolemmal membrane remained unchanged, the proportion of lipids present in MLV-SL and extruded from the sarcolemma was calculated. The data in Table 4.3 show that the estimated amount of lipid extruded from the sarcolemma and packed in multilamellar vesicles tended to increase during the ischemic insult. The changes observed in cardiac tissue, reperfused after 60 and 90 minutes of preceding ischernia, reached the level of significance. Up to $5 \%$ of sarcolemmal lipids were extruded and stored in the multilamellar vesicles.

No signifficant difference in the periodicity (lamellar spacing) of the multilamellar vesicles was found between MLV-SL, MLV-MITO or MLV-LIPID, nelther within the various groups, nor between the control group $\left(30^{\prime} \mathrm{L}\right)$ and the severely ischemic and reperfused group $\left(30^{\prime} \mathrm{L}+90^{\prime} \mathrm{I}+30^{\prime} \mathrm{R}\right)$ (Table 4.2).

4.3.4. Correlation between ischemic injury scoring and the number of multilamellar extrusions.

A significant $(P<0.05)$ relation was observed between the ischemic injury score on the one hand, and the number of MLV-SL $\left(R_{S}=0.86\right)$. MLV-MITO $\left(R_{s}=0.64\right)$ and MLV-TOTAL minus MLV-LIPID $\left(R_{S}=0.71\right)$ on the other, using Spearman's rank correla- 

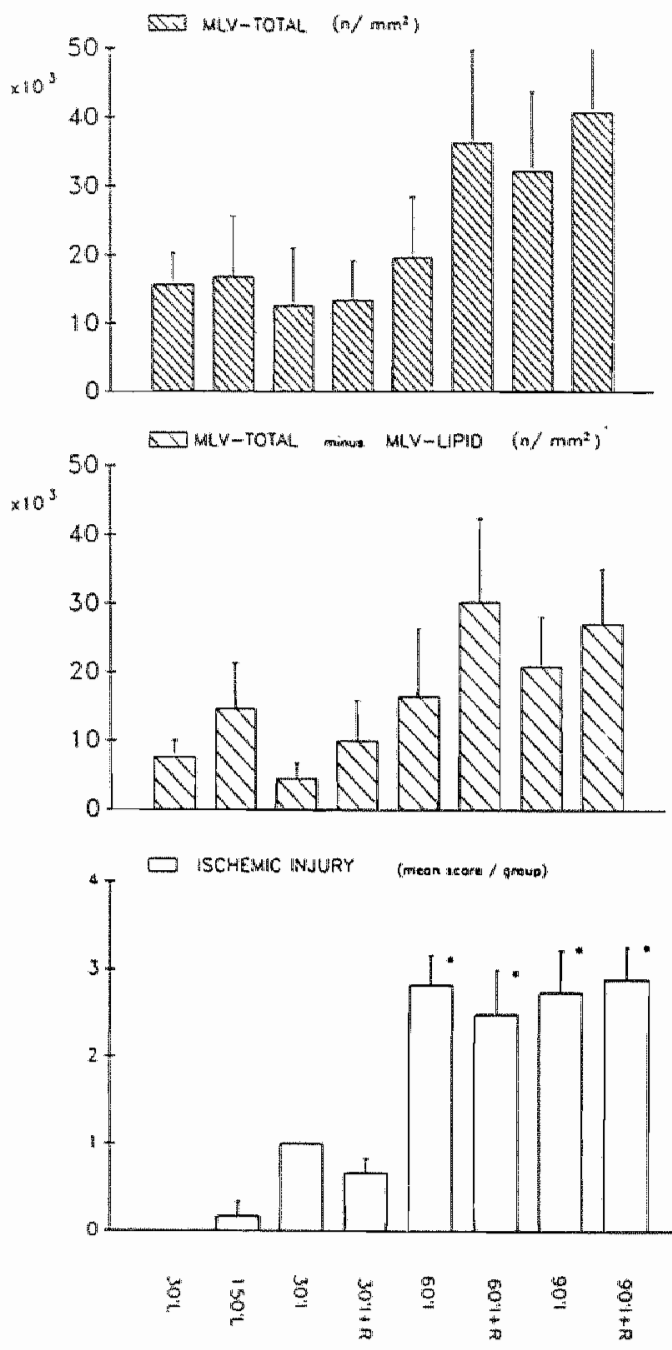

Figure 4.2. Bar diagroms show'mg the newat manber of millitantellar vestoles per etperimonat group and stardard error of the meon.

(a) MLVTOTAL, sum of mulihamellay vesicles anmded from the sancolemwa. mitochandria and lipid dropless, and moilnclassified multilamellar nesicles fupper graph).

(b) MLV TOTAL MEMS MLV-LIVID (maidolle graphl. * indicanss a significant difurence $(P<0.05)$ with the 302 control group (Mann-Whituey U-Hesi).

(c) Bar diagram of the mean per growp and standard error of the miean of myocardial ischemic intwry score (lower graphl.

$L=$ Largendorf penfision, $l=15$ chemia and $R=$ Reperfiesion fas Langendorth. * indicanes a signifioam difference $\left(p^{p}<0.05\right)$ whith the $30^{\prime \prime} \mathrm{L}$ and $150^{\circ} \mathrm{L}$ control gromps (Momn-Mhirney Uatest).

tion $\left(R_{s}\right)$. The significant increase in the ischemic injury scoting was also related in time ( 60 minutes of ischemia) to the significant increase of MLV-MITO. 


\subsection{DISCUSSION}

Multilamellar vesicles can be found in both endothelial cells and candiomyocytes. In the myocytes the multilamellar vesicles are either extruded from the sarcolemma or mitochondrial membranes, or located adjacent to lipid droplets. Morphometric analysis revealed that in the order of $5 \%$ of the membrane lipids originally present in the sarcolemma is packed in the MLV-SL of cells damaged by ischemia and reperfusion. Morphological features of multilamellar vesicles, such as size (approximate area of 0.05 $\mu \mathrm{m}^{2}$ ) and periodicity of the lamellae (approximately $5.6 \mathrm{~nm}$ ) are not influenced by ischemia and reperfusion, indicating that the experimental condition and the localization are not of influence on the morphological architecture of the multilamellar extrusions observed.

TABLE 4.1. Mean size of MLV-TOTAL (all observed multilamellar vesicles) per experimental group.

\begin{tabular}{llll} 
EXPERIMENTAL & \multicolumn{3}{c}{ MLV-TOTAL SIZE $\left(\times 10^{-3} \mathrm{\mu m}^{2}\right)$} \\
CONDITIONS & MEAN & SEM & $\mathrm{n}$ \\
$30^{\circ} \mathrm{L}$ & 29.5 & 3.2 & 38 \\
$150^{\prime} \mathrm{L}$ & 43.1 & 8.7 & 22 \\
$30^{\prime} \mathrm{L}+30^{\prime} \mathrm{I}$ & 48.5 & 5.1 & 25 \\
$30^{\prime} \mathrm{L}+30^{\prime} \mathrm{I}+30^{\circ} \mathrm{R}$ & 74.2 & 13.1 & 19 \\
$30^{\prime} \mathrm{L}+60^{\circ} \mathrm{I}$ & 40.8 & 7.1 & 32 \\
$30^{\prime} \mathrm{L}+60^{\prime} \mathrm{I}+30^{\circ} \mathrm{R}$ & 51.0 & 6.0 & 55 \\
$30^{\prime} \mathrm{L}+90^{\prime} \mathrm{I}$ & 45.1 & 6.6 & 54 \\
$30^{\prime} \mathrm{L}+90^{\prime} \mathrm{I}+30^{\prime} \mathrm{R}$ & 43.8 & 4.6 & 80 \\
& & &
\end{tabular}

$L=$ Langendorf peffusion, $\mathbb{L}_{2}=$ Ischemia, $\mathrm{R}=$ Reperfusion (same conditions as $\mathrm{L}$ ). Data are presented as means $\left(x 10^{-3} \mathrm{~mm}^{2}\right)$ per experimental group and standard error of the mean (SEM). a is the number of multilamellar vesicles examined. The size of the multibmellar vesicles in the first group (30L) is significantly smaller (Student T-test, unpaired) than in the other groups (except $30^{*} \mathrm{~L}+60^{\prime} \mathrm{I}$ ). 

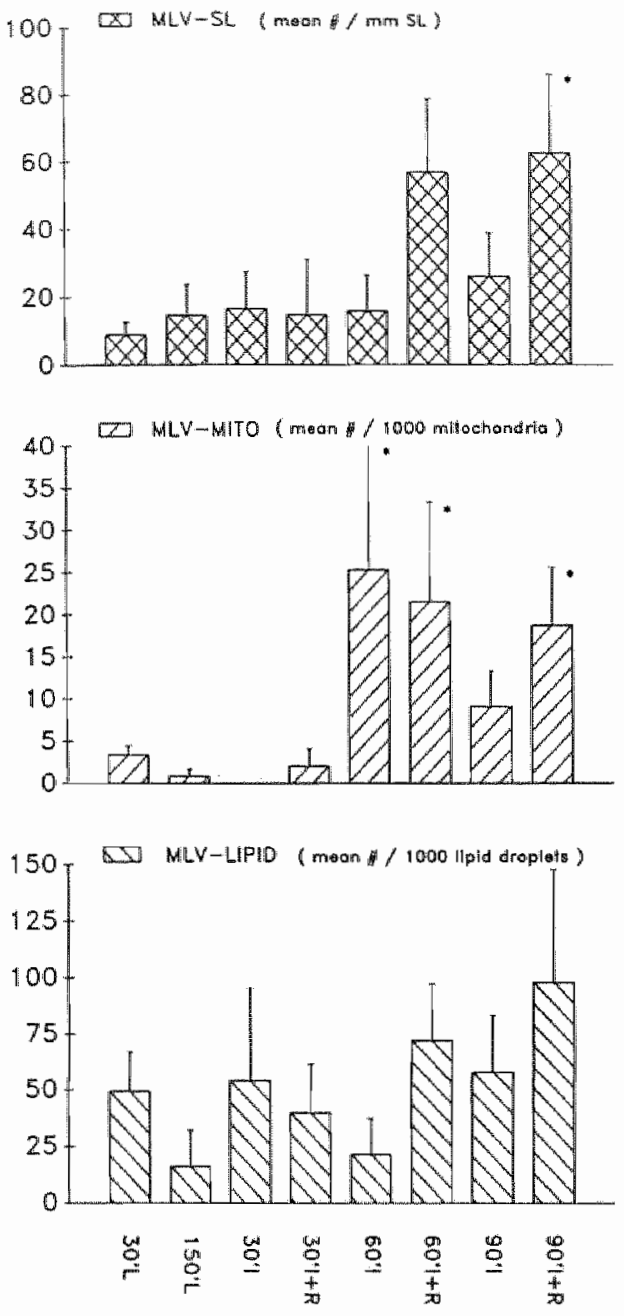

Figure 4. 3. Bar diagroms showing the wath

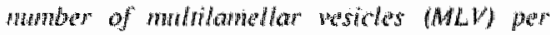

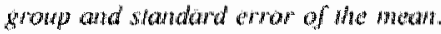

(a) Disfribution of the men number pret

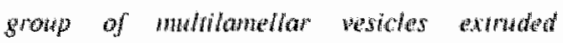
from the sacolema (MLV-SLU), expressed as Whe nowore of mullilamellar vesicles per millimeter sancolenma (nppar" graph).

(b) Mean manbers per group of mulfilanellar vesicles extraded from moltochoudtia (MLV-MINO), expressed an nomber $\left(x 10^{-3}\right.$ ) per marochondrion (middle traph).

(c) Mean nwmbers per growp of mulitametion vesicles adjoining fipid droplets MLV. LIPDD), cipressed in number $\left(x 10^{-3}\right)$ pert lipial drople (lower graph).

$L=L$ angendorff perfusion, $t=1$ schentia and $R=$ Reperfusion (Langendorf). He indicates a sigmifican difference $(P<0.05)$ with the $30 ' \mathrm{~L}$ and 150'L Conno' growps (Mann-Whimey $U-(t ; s)$.

The number of multilamellar vesicles associated with intracellular lipid droplets (MLV-LIPID) appeared to be independent of the (patho)physiological state of the cell.

The number of multilamellar vesicles extruded from mitochondria (MLV-MITO), however. increases significantly after periods of ischemia exceeding 60 minutes. Unlike MLV-MITO the number of multilamellar vesicles extruded from the sarcolemma (MLV- 
SL) is only slightly increased in the ischemic tissue. Restoration of flow, following an ischemic period of at least 60 minutes, induced the formation of a significant number of MLV-SL. This observation underlines the presence of specific reperfusion induced damage $(13,77)$ at the level of the membrane enclosing the previously ischemic cardionyocyte. Per experimental group the number of multilamellar vesicles observed appears to parallel the semi-quantitative scoring of myocardial ischemic injury.

In the present study tannic acid based fixation was used to visualize multilamellar vesicles. Tannic acid has been proposed to improve the preservation of (saturated) phosphatidylcholines (134). Recently we have shown that tannic acid not only reduces the extraction of phospholipid, but also induces an instant aggregation and formation of multilamellar structures, when added to sonicated unilamellar vesicles, specifically containing phosphatidylcholines (194. 194, 195). These experiments were performed with synthetic phospholipids (DMPC, DOPC) and purified phospholipids, either commercially obtained (PS, PE) or extracted from rabbit or rat hearts (complex composition of phospholipids). Based on these experiments, it was concluded that the interaction of tannic acid with phospholipids in the bilayer arrangement influences the electrostatic or hydration repulsive forces between the lipid bilayers and, hence, facilitates aggregation and/or fusion of unilamellar vesicles and the subsequent formation of multilamellar vesicles. The ability of tannic acid to induce lamellar structures has also been observed in myeloma cells (178) and in plant tissue (251).

At present we assume that in the myocardium tannic acid induces the formation of multilamellar vesicles, thereby reflecting changes in the accessibility of tannic acid to membrane phospholipids with a choline headgroup (phosphatidylcholine, the plasmalogen phosphatidalcholine, sphingomyelin and also lysomphosphatidylcholine). The accessibility of phospholipids may be changed under conditions of ischemia and reperfusion. For example, accumulation of degradation products, such as lyso-phospholipids and fatty acids, in the lipid bilayer may add to instability of the membranes (127). Furthemore, ischemia induced alterations in the interaction of membrane lipids and the glycocalyx or the cytosketeton may render the membrane lipids more susceptible to tannic acid interaction.

There is increasing evidence that alterations in myocardial membrane phospholipids play an important role in the pathogenesis of ischemic cell injury $(43,54,198$, 
199. 264). However, in myocardial ischemia phospholipid breakdown is a relative late event ( $1-3 \mathrm{~h})$, indicating that more subtle membrane alterations are involved in the onset of irreversible injury.

It has been shown that in the sarcolemma of severely ischemic dog hearts small breaks or discontinuities develop at or about the time that the ischemic injury becomes irreversible (121). In our experiments we have not observed any distuptions or breaks in the sarcolemma, although some hearts, which had been exposed to 90 minutes of ischemia and 30 minutes reperfusion, showed severe and possibly irreversible ischemic cell damage. The observed increased number of multilamellar vesicles, extruded from the non-disrupted sarcolemma, may be a reflection of more subtle functional changes in the myocyte membrane. This is supported by the finding that enzyme leakage can be observed under these circumstances (233, see also Chapter 5), indicating that rupture of the sarcolemma is not a prerequisite for the release of cellular enzymes and the onset of irreversible cell injury.

It is noteworthy to mention that in hearts rendered ischemic for 30 minutes multilamellar vesicles were also observed extruded from vascular endothelial cells, which is in agreement with the biochemical observation of early damage of vascular endothelium during cardiac ischemia (109).

TABLE 4.2. Periodicity of multilamellar vesicles extruded from sarcolemma (MLV-SL), mitochondria (MLV-MITO) or lipid droplets (MLV-LIPID) in control hearts $\left(30^{\circ} \mathrm{L}\right.$ ) and in ischemic, reperfused hearts $\left(30^{\prime} L+90^{\prime} I+30^{\prime} R\right)$.

\begin{tabular}{|c|c|c|c|c|c|c|}
\hline & \multicolumn{6}{|c|}{ PERIODICITY OF MLV"s (in nn) } \\
\hline & \multicolumn{3}{|c|}{ CONTROL $\left(30^{8} \mathrm{~L}\right)$} & \multicolumn{3}{|c|}{$30^{\prime} \mathrm{L}+90^{\prime} \mathrm{I}+30^{\prime \prime} \mathrm{R}$} \\
\hline & MEAN & SEM & $\underline{n}$ & MEAN & SEM & $\underline{n}$ \\
\hline $\mathrm{MLV}-\mathrm{SL}$ & 5.42 & 2.8 & 6 & 5.62 & $\mathbb{1 1 . 3}$ & 17 \\
\hline MLV $\backsim$ MITO & 5.72 & 4.2 & 4 & 5.71 & 2.0 & 10 \\
\hline MLV-LIPID & 5.39 & 2.5 & 5 & 6.01 & 2.2 & 10 \\
\hline
\end{tabular}

Data ate presented as mean distance between the lamellate and standard error of the mean (SEM). $n$ is the number of vesicles examined. $L=$ Langendorff perfusion. 1 = Ischenia, $R$ Repertusion (same conditions as L). 
Since the lipid droplers in the cardiac myocytes mainly consist of triglycerides and do not have a phospholipid bilayer, the formation of multilamellar vesicles adjacent to these organelles under influence of tannic acid must be of a different nature.

The question can be raised as to whether the multilamellar vesicles represent the "in wivo" siluation or have to be considered as a fixation phenomenon. Lamellar vesicles have been observed in ischemic and/or reperfused myocardium by freezefractioning without the use of tannic acid (164). Other studies have shown that tannic acid induces the formation of multilamellar structures $(178,191,194,195,251)$. Since Post and co-workers $(164,165)$ did not quantitate the observed multilamellar vesicles, as in the present study, it cannot be excluded that multilamellar vesicles may at least partially be induced by tannic acid. In this light multilamellar vesicles do not necessarily reflect the "in vivo" situation in a quantitatiwe sense when tannic acid based fixation is used. At present we therefore consider the use of tannic acid based fixation as a "cytochemical" method to demonstrate membrane instability.

The precise mechanism underlying ischemic and reperfusion induced cellular damage is incompletely understood $(77,121,242)$. Several mechanistic explanations have been given for the ischemia and reperfusion induced membrane changes. Ulirastructural impairment following depletion of cellular high energy phosphates. (ATP and creatine phosphate) and/or increases in AMP and adenosine (234) may result in plasma membrane or cytoskeleton disonders, but not in overt sarcolemmal rupture or the formation of subsarcolemmal blebs (179). Fragmentation of previously weakened plasma membranes may result from stress on the membrane, as exerted by physical lorces, such as intracellular edema or contraction induced mechanical stress $(121,242)$. Alternatively, ischemia andior reperfusion induced changes can be explained by the physico-chemical behavior of the phospholipid constituents of the plasma membrane. Increased levels of $\mathrm{Ca}^{2}$ ions and low $\mathrm{pH}$ can promote phase transition, leading to lateral phase separation and intramembranous particle aggregation (250).

All of these changes in the plasma membrane architecture can either directly lead to the formation of multilamellar extrusions or render the membrane phospholipids more susceptible to tannic acid interaction and the secondary induction of multilamellar extrusions during fixation. However one has to realize that in both 
TABLE 4.3. Percentage of lipid in multilamellar extrusions, expressed as the ratio of length of membrane-lamellae in multiamellat vesicles extruded from the sarcolemma (MLV-SL) and length of sarcolemma examined.

\begin{tabular}{llll}
\hline EXPERIMENTAL & \multicolumn{3}{c}{ \% of lipid in MLV-SL } \\
CONDITIONS & $\frac{\text { MEAN }}{30^{\prime} \mathrm{L}}$ & $\frac{\text { SEM }}{0.73}$ & $\frac{1}{5}$ \\
$150^{\prime} \mathrm{L}$ & 0.38 & 5 \\
$30^{\circ} \mathrm{L}+30^{\prime} \mathrm{I}$ & 1.25 & 0.77 & 3 \\
$30^{\circ} \mathrm{L}+30^{\prime} \mathrm{I}+30^{\prime} \mathrm{R}$ & 1.44 & 0.93 & 4 \\
$30^{\circ} \mathrm{L}+60^{\circ} \mathrm{I}$ & 1.28 & 1.28 & 3 \\
$30^{\circ} \mathrm{L}+60^{\prime} \mathrm{I}+30^{\prime} \mathrm{R}$ & 1.37 & 0.91 & 3 \\
$30^{\circ} \mathrm{L}+90^{\prime} \mathrm{I}$ & $4.74^{*}$ & 1.81 & 3 \\
$30^{\circ} \mathrm{L}+90^{\prime} \mathrm{I}+30^{\prime} \mathrm{R}$ & 2.22 & 1.09 & 4 \\
& $5.72^{*}$ & 2.04 & 5 \\
\hline
\end{tabular}

Data are presented as means per experimental group and standard error of the mean (SEM). expressed in percentages. $\mathbf{n}$ is the number of rabbits examined per experimental group. $L$ is Langendorff perfusion, $I$ is ischemia, $R$ is reperfusion (same condition as $L$ ) indicates a significant $(\mathrm{P}<0.05)$ difference with the $\left(30^{\circ} \mathrm{L}\right)$ control group, using the Manm-Whitmey U-test.

cases the multilamellar vesicles are an indication of membrane damage.

It is concluded that the multilamellar vesicles may reflect subtle, ischemia induced alterations in membranes. Determination of the number of nultilamellar extrusions might be useful to quantitate ischemia and reperfusion induced myocardial (membrane) injury and, hence. to examine the influence of metabolic alterations on membrane integrity. In this respect it is of interest mentioning that a significant accumulation of fatty acids has been observed in dog and in rat hearts alter 45-60 minutes of ischemia $(44,167,234)$. These findings suggest a relation in time between the increase in the number of multilamellar extrusions and the accumulation of fatty acids. Whether there is a causal relation remains to be established. 


\subsection{SUMMARY}

The present study was designed to investigate The influence of the duration of ischemia and reperfusion on the induction of multilamellar vesicles in isolated rabbit hearts and to examine whether these multilamellar extrusions can be used as a parameter to quantitate ischemic (membrane) injury.

Multilamellar vesicles were found in both endothelial cells and myocytes. The number, size, architecture and (sub-)cellular location of multilamellar vesicles in myocytes were analyzed in more detail. A limited number of multilamellar vesicles was observed after 30 or 150 minutes control perfusion. The total number of multilamellar vesicles observed in myocytes, in general, and the number of multilamellar vesicles extruded from mitochondria, in particular, were found to be increased in hearts which had been ischemic for at least 60 minutes. The number of multilamellar vesicles extruded from the sarcolemma was only increased in hearts that had been reperfused after minimally 60 minutes of ischemia. The number of multilamellar vesicles adjacent to lipid droplets varied remarkably and was found to be independent of the duration of ischemia. The observed multilamellar vesicles were all very similar in size and in periodicity of the lamellae.

Ischemic injury, as scored on the basis of various morphological parameters, appeared to be significantly related to the number of multilamellar vesicles extruded from sarcolemma or mitachondria. It is therefore suggested that the number of multilamellar vesicles can be used to quantitate ischemic (membrane) injury. 


\section{CHAPTER 5}

CORRELATION IN TIME BETWEEN THE INCREASE OF MULTILAMELLAR VESICLES AND METABOLIC ALTERATIONS IN ISCHEMIC AND REPERFUSED RABBIT HEARTS

Based on: A.H.G.J. Schrijvers, M.J.M. de Groot, V.V.Th Heijnen, G.J. Van der Vusse, P.M. Frederik, R.S. Reneman (1989) J Mol Cell Cardiol (provisionally accepted for publication). 


\subsection{INTRODUCTION}

There is increasing evidence that the development of sarcolemmal dysfunction is of considerable importance in the pathogenesis of ischemia and reperfusion induced myocardial cell injury $(50,72,127,243)$. The loss of membrane integrity is thought to be associated with degradation of phospholipids $(40,43,196,199,221,264)$ as among others indicated by the accumulation of fatty acids in the ischemic myocardium (44. 167, 234, 237, 240). These fatty acids most likely originate from membrane phospholipids, since a major component of the accumulated fatty acids is arachidonic acid, a fatty acid known to be almost exclusively present in cellular phospholipids.

Relatively little is known about the effects of reperfusion on myocardial lipid metabolism. It has been reported that the tissue levels of acylcarnitine and acyl-CoA return to control levels in reperfused hearts $(150,159)$, suggesting normalization of fatty acid oxidation and/or rapid reincorporation of these acyl-moieties into the phospholipid or triacylglycerol pool. On the other hand phospholipid homeostasis does not seem to recover upon reperfusion, since the tissue content of phospholipids significantly decreases during reperfusion $(34,54,158)$. Moreover, the myocardial content of arachidonic acid remains elevated and accumulation may even proceed in reperfused hearts $(34,234,241)$.

Beside metabolic studies considerable effort has been made to study morphological alterations during ischemia and reperfusion $(129,259)$. Rupture of the sarcolemma during ischemia and/or perfusion is a clear functional and morphological indication of irreversible membrane damage $(88,116,119)$. Recently, several reports described more subtle membrane alterations, such as the extrusion of multilamellar. liposome-like structures from the sarcolemma and mitochondrial membranes in ischemic and reperfused hearts $(164,165,189,193)$. These multilamellar vesicles are thought to reflect ischemia and/or reperfusion induced membrane instability.

It was the aim of the present study to investigate the time course of multilamellar vesicle formation and the relation between the increase in the number of multilamellar extrusions on the one hand and 1) the tissue content of ATP, 2) the breakdown of phospholipids and triacylglycerols, and 3) the accumulation of fatty acids in general and arachidonic acid in particular on the other. To this end the time 
interval of ischemia was varied and the effects of reperfusion were examined. Lactate dehydrogenase release was measured during the period of repertusion and used as an indicator of cell damage.

\subsection{MATERIAL AND METHODS}

\subsubsection{Preparation of the hearts.}

The experiments were performed on male New Zealand White rabbits. After the hearts of the rabbits were removed, as described in detail in chapter 4.2 .1 , they were attached to the aortic cannula of the perfusion system. After attachment to the perfusion system was completed the hearts were perfused with a modified Tyrode"s solution, described in more detail in Chapter 4.2.2, under various experimental conditions, as described in Chapter 4.2.3.

\subsubsection{Fixation and tissue processing.}

At the end of the experiments biopsies were taken near the apical side of the left ventricle for biochemical determinations. These biopsies were immediately freezeclamped with Wollenberger clamps, pre-cooled with liquid nitrogen. Thereafter the hearts were fixed by perfusion (at a perfusion pressure of $8 \mathrm{kPa}$, at $25^{\circ} \mathrm{C}$ ) with a combination of $2.5 \%$ glutaraldehyde (Bjorad; A2523) and $2 \%$ tannic acid (Mallinckrodt Inc; AR 1764 ) in $20 \mathrm{mM}$ phosphate buffered physiological saline (PBS, pH 7.2) for 10 minutes. Midwall biopsies (about $1 \mathrm{~mm}^{3}$ ) were cut from the left ventricle and fixed by immersion at room temperature for 2 hours in the same fixative. Tissue specimens were further processed for electron microscopic examination, as described in Chapter 4.2.4.

\subsubsection{Morphometric analysis.}

For each experiment 10 micrographs were taken randomly from one grid at the 
same magnification. Micrographs were analyzed morphometrically (Kontron: MOPvideoplany to establish the number of multilamellar vesicles.

The number of multilamellar vesicles extruded from the sarcolemma (MLV-SL) was expressed as the mean number of multilamellar vesicles per mm sarcolemma. The number of multilamellar vesicles extruded from mitochondria (MLV-MITO) and adjoining lipid droplets (MLV-LIPID) was expressed as the mean number $\left(\mathrm{x} 10^{-3}\right)$ per mitochondrion and lipid droplet, respectively. (MLV-TOTAL) was considered to be the sum of multilamellar vesicles observed per experiment "extruded from the sarcolemma, mitochondria or located adjacent to or in lipid droplets and of multilamellar vesicles of unidentifted origin that could not be classified with certainty to any of the previously mentioned groups. For a more detailed description see Chapter 4.2.5.

\subsubsection{Release of lactate dehydrogenase (LDH).}

During reperfusion the coronary effluent was sampled at regular time intervals for subsequent analysis of enzyme release. $\mathrm{LDH}$ release was taken as an index of the loss of cellular integrity. The effluent samples could be stored for several months without a significant loss of LDH activity by adding albumin (final concentration of $3 \% \mathrm{w} / \mathrm{v})$ to the effluents $(211)$. LDH activity was measured spectrophotometrically with the use of a Cobas-Bio (Hoffman-LaRoche) autoanalyzer (17).

\subsubsection{Analysis of tissue lipids.}

The freeze-clamped tissue biopsies were pulverized in an aluminum mortar, tissue powder was transferred to a test tube and the lipids were extracted by addition of a mixture of chloroform/methanol (2/1 by volume), as described in more detail in Chapter 3.2 .2 .

5.2.6. Analysis of the tissue contents of ATP, glycogen and lactate.

Another part of the deeply frozen tissue was freeze-dried overnight at $-30^{\circ} \mathrm{C}$ and extracted at $-15^{\circ} \mathrm{C}$ in a mixture of perchloric acid $(3.0 \mathrm{M})$ and dithiothreitol $(5 \mathrm{mM})$. 
After centrifugation (for 5 minutes at $1200 \mathrm{~g}, 4^{\circ} \mathrm{C}$ ) a known amount of the supernatant was removed and neutralized with $\mathrm{KHCO}_{3}$, whereas the pelleted residue was stored at $-20^{\circ} \mathrm{C}$ for subsequent determination of glycogen. The salt-precipitate of the neutralized extract was pelleted by centrifugation (for 60 minutes at $1200 \mathrm{~g}, 4^{\circ} \mathrm{C}$ ) and the clear supernatant was stored at $-80^{\circ} \mathrm{C}$ until further analysis. The content of ATP was determined by high performance liquid chromatography (Varian Vista $5500 \mathrm{HPLC}$ ), essentially according to the method of Wynants and Van Belle (263). Lactate and glycogen were determined as previously described (238).

\subsubsection{Statistical analysis.}

All data are presented as mean values and standard error of mean (SEM) per experimental group. Differences between groups were analyzed for statistical significance using the Mann-Whitney U-test. P-values less than 0.05 were considered to be statistically significant. Spearman's coefficient of rank correlation $\left(\mathbf{R}_{\mathbf{s}}\right)$ was used to estimate the strength of the relation between two variables. Correlations between the morphological and biochemical parameters were very poor when individual data (per experiment) were considered. This was most likely due to the fact that biochemical and ultrastructural data could not be obtained in the same piece of tissue. In adclition. the size of the samples for ultrastructural investigation are many times smaller than those for lipid analysis, which may lead to sampling errors. Therefore, it was decided to process data as mean values per experimental group when possible relations. between the parameters were studied.

\subsection{RESULTS}

\subsubsection{Morphology.}

Multilamellar vesicles were found in myocytes extruded from sarcolemma (MLVSL), mitochondrial membranes (MLV-MITO) and lipid droplets (MLV-LIPID) (Fig. 5.1). The number of multilamellar extrusions varied under the different experimental 


\section{Chapters}
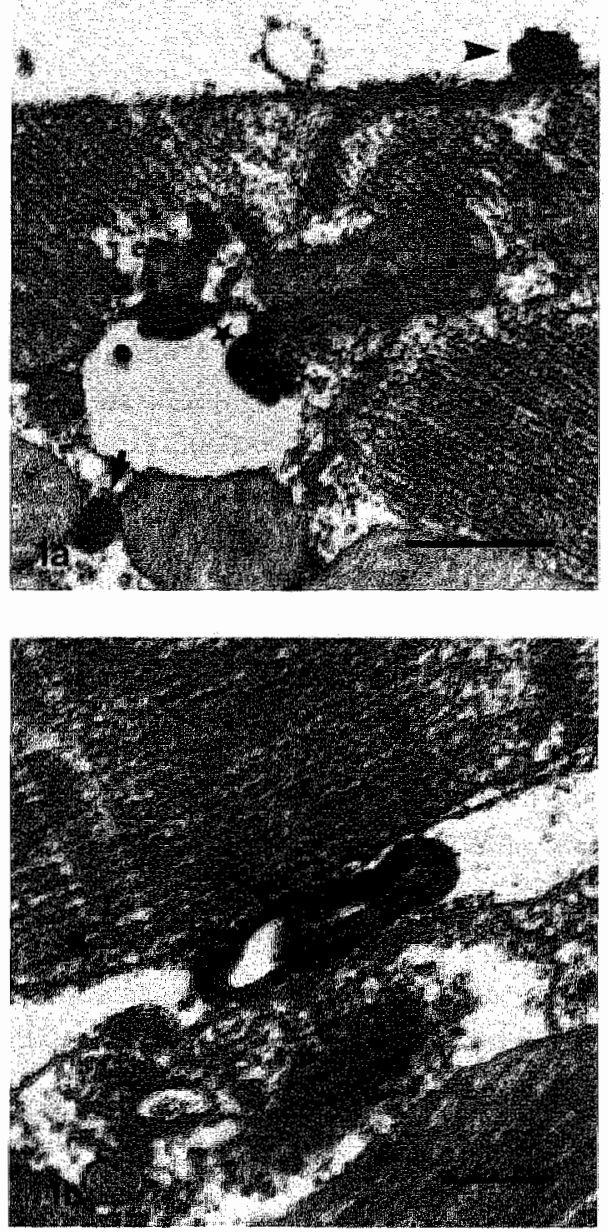

Figure 5.1. Electronmicrographs demonstrating multilantilar vesiches extruded from differem organtelles in severely ischemic and reperfused hears $\left(30 \mathrm{~L}+90^{\circ} \mathrm{H}+30^{\prime} \mathrm{R}\right)$.

(a) Mulillanellar wesicles are exiruded from the sarcolemma (MLV-SL, arrowhead) or mitochondrial membrastes (MLV-MTTO, arrow) and in or adjacent to lipid dropless (MLV-LUPID, asterisk). The bar represents 0.5 pm.

(b) A detail of a mullitanellar vesicle exiruded from the sarcolemma (MLV-SL). The bar represents 0.2 um.

conditions. In general the relative distribution of multilamellar vesicles was as follows: about 25-30\% of the observed multilamellar vesicles were extruded from the sarcolemma; $25-30 \%$ were extruded from mitochondrial membranes and $40-50 \%$ of the multilamellar vesicles were extruded from or adjacent to lipid droplets.

The number of multilamellar vesicles observed generally increased after prolonged ischemia, whereas reperfusion caused a further increase (Table 5.1). No significant difference could be observed between the number of multilamellar vesicles in control tissue analyzed after 30 or 150 minutes perfusion. The mean number of MLV-SL was 
doubled after 30 and 60 minutes of ischemia as compared with 30 minutes control perfusion. The mean number of MLV-MITO was drastically increased in tissue subjected to 60 and 90 minutes of ischemia (Table 5. 1).

The mean numbers of MLV-SL and MLV-MITO were drastically increased in hearts reperfused after ischemic periods of 60 and 90 minutes. The number of MLVLIPID did not change significantly under the various experimental conditions. The total number of multilamellar vesicles observed, without the multilamellar vesicles extruded from or adjacent to lipid droplets (MLV-TOTAL minus MLV-LIPID), showed a gradual increase after prolonged ischemia and a further increase after reperfusion, as compared with the corresponding ischemic group without reperfusion (Table 5.1).

The size (approximately $0.22 \mu \mathrm{m}$ in diameter) and the lamellar repeat distance (approximately $5.65 \mathrm{~nm}$ ) was similar for all multilamellar vesicles, irrespective of location and experimental condition.

\subsubsection{Metabolic alterations.}

The tissue content of ATP, about $17 \mu \mathrm{mol} / \mathrm{g}$ dry weight in controll hearts, gradually decreased with the duration of the ischemic period. This decrease was significant in all hearts rendered ischemic. The level of ATP did not recover after restoration of flow following 30 and 60 minutes of ischemia. A slight restoration of ATP levels was observed in cardiac tissue reperfused after 90 minutes of flow cessation (Fig. 5.2b).

The tissue content of fatty acids, approximately $430 \mathrm{nmol} / \mathrm{g}$ dry weight in control hearts, was significantly (about 3-fold) increased after 60 and 90 minutes of ischemia. Tissue levels of fatty acids tended to increase further after restoration of flow. All reperfused hearts showed a significant accumulation of fatly acids when compared with the control groups (Table 5.2).

The level of arachidonic acid, a fatty acid almost exclusively present in phospholipids, was approximately $25 \mathrm{mmol} / \mathrm{g}$ dry weight in control hearts and tended to increase in cardiac tissue made ischemic for 30 minutes. The arachidonic acic content was drastically increased (about 5-fold) after 60 and 90 minutes of ischemia, the latter 
TABLE S.1. The number of multilamellar vesicles extruded from different organelles under various experimental conditions.

\begin{tabular}{|c|c|c|c|c|c|}
\hline & $n$ & $\frac{M L Y-S L}{(f / \mathrm{mm} \text { SL })}$ & $\frac{\text { MLV-MITO }}{\left(1+10^{-3} / \text { mito }\right)}$ & $\frac{\text { MLV-LIPID }}{\left(* 10^{-3} / \text { ip }\right)}$ & $\frac{M L V-T-L}{(*)}$ \\
\hline $30 \mathrm{~L}$ & 5 & $8.8 \pm 3.7$ & $3.4 \pm 1.0$ & $49.2 \pm 17.6$ & $3.4 \pm 0.9$ \\
\hline $150^{\circ} \mathrm{L}$ & 3 & $14.6 \pm 9.0$ & $0.8 \pm 0.8$ & $16.1 \pm 16.1$ & $4.1 \pm 2.4$ \\
\hline $30^{\circ} \mathrm{L}+30^{\prime} I$ & 4 & $16.7 \pm 10.8$ & 0.0 & $54.0 \pm 41.4$ & $2.3 \pm 1.1$ \\
\hline $30^{\prime} L+30^{\prime} I+30^{\prime} R$ & 3 & $14.8 \pm 16.1$ & $2.0 \pm 2.0$ & $39.0 \pm 21.8$ & $4.7 \pm 2.7$ \\
\hline $30^{\prime} \mathrm{L}+60^{\prime} \mathrm{II}$ & 3 & $15.9 \pm 10.5$ & $25.3^{*} \pm 15.1$ & $21.5 \pm 15.7$ & $9.0 \pm 5.6$ \\
\hline $30^{\prime \prime} L+60^{\prime} I+30^{\prime \prime} R$ & 3 & $56.9 \pm 21.7$ & $21.5^{*} \pm 11.9$ & $72.1 \pm 25.0$ & $15.3 \pm 6.2$ \\
\hline $30^{\prime \prime} \mathrm{L}+90^{\circ} \mathrm{I}$ & 4 & $26.0 \pm 12.7$ & $9.1 \pm 4.2$ & $58.1 \pm 25.1$ & $8.3 \pm 2.8$ \\
\hline $30^{\prime} \mathrm{L}+90^{\prime} \mathrm{I}+30^{\prime} \mathrm{R}$ & 5 & $62.4^{\text {闻 }} \pm 23.4$ & $18.7^{*}+6.9$ & $97.9 \pm 49.7$ & $10.2^{3} \pm 2.5$ \\
\hline
\end{tabular}

The data are presented as mean values and standard error of the mean (SEM) per experinental group. MLV-SL $=$ Multilamellar vesicles extruded from the sarcolemma. MLV-MITO = Multilamellar wesicles extruded from mitochondrial membranes. MLV-LIPID $=$ Multilamellar vesicles extruded from or adjacent to lipid droplets. MLV-T-L = Total number of multilanellar vesicles minus the multiamellar vesicles extruded from or adjacent 10 lipid droplets. $n=$ number of experiments per group, $\#=$ mean number per group, mito $=$ mitochondrion and lip $=$ lipid droplet. L. I and $\mathrm{R}$ refer to Langendorff perfusion, Ischemia and Reperfusion (Langendorff), respectively. * indicates a significant $(\mathbb{P}<0.05)$ difference with the control perfused groups, using the MannWhitney U-iest.

increase being significant. Arachidonic acid was significantly increased in all hearts that were reperfused, as compared with both control groups, i.e. 30 or 150 minutes of normoxic perfusion (Fig. 5.2a).

The amount of triacylglycerols, about $90 \mu \mathrm{mol} / \mathrm{g}$ dry weight in control hearts. varied strongly from heart to heart, but no significant difference was observed under the various experimental conditions, as compared with both control groups (Table 5.2).

The total phospholipid pool, approximately $210 \mu \mathrm{mol}$ fatty acyl moieties/g dry weight in control hearts, did not change significantly in any of the experimental groups (Table 5.2). 

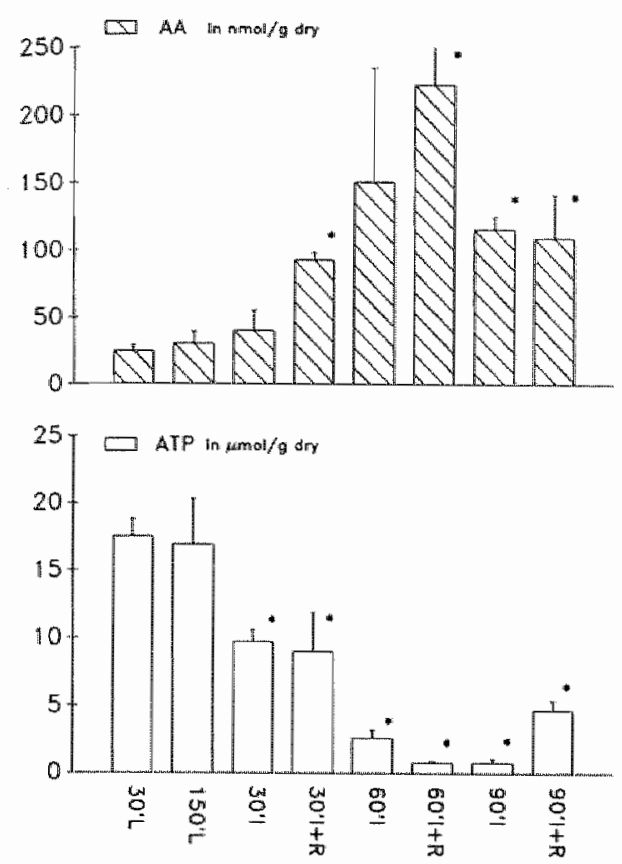

Figure 52. Dan hagnams showng the

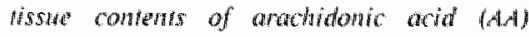
and atchosing (riphospharite (ATP).

(a) The tissme conrent of AA (miean $t$ SEMI) mcreases with prolonged duranon of the inchennic period * indicares a stignificom $(P<0.05)$ diflerence wh the $10 \mathrm{~L}$ and 150 ' control groups whing the ManmWhilnay U-resi (apper guaph).

(b) The riswe contern of A7p (medar 4 SEM) gradinally decreases with the duration of ischemia * indicates a sigufican $(P<0.05)$ difference with the $30 \mathrm{~L}$ and $150 \mathrm{~L}$ control groups fexcepr for the $30 \mathrm{~L}+30^{\prime \prime} \mathrm{T}+30^{\prime} \mathrm{R}$ group only with the $30^{\mathrm{L}} \mathrm{L}$ control gronpl. wsing the Month. Whilhey Unest (lower graph).

It should be noted that the pronounced increase in the tissue level of arachidonic acid, a major constituent of membrane phospholipids, likely reflects degradation of phospholipids. The observation that the accumulation of arachidonic acid occurs in the nmolar range explains why no concomitant net degradation of the total phospholipid pool ( $\mu$ molar range) could be observed.

The release of LDH, measured in the effluent of reperfused hearts and expressed as Units per 30 minutes of reperfusion, was significantly $(P<0.05$, Mann-Whitney $U$. test) increased in hearts reperfused after 60 minutes $(26.6 \pm 10.9$, mean $\pm S E M, n=3$ ) and 90 minutes $(37.6 \pm 5.0, n=6)$ of ischemia as compared with the minor release of LDH ( $5.4 \pm 2.1, n=3$ ) from hearts reperfused after 30 minutes of ischemia.

The tissue content of glycogen, approximately $225 \mu \mathrm{mol} / \mathrm{g}$ dry weight in control hearts, gradually decreased with prolonged duration of the ischemic period. The content of glycogen was significantly higher in hearts reperfused after 90 minutes of 

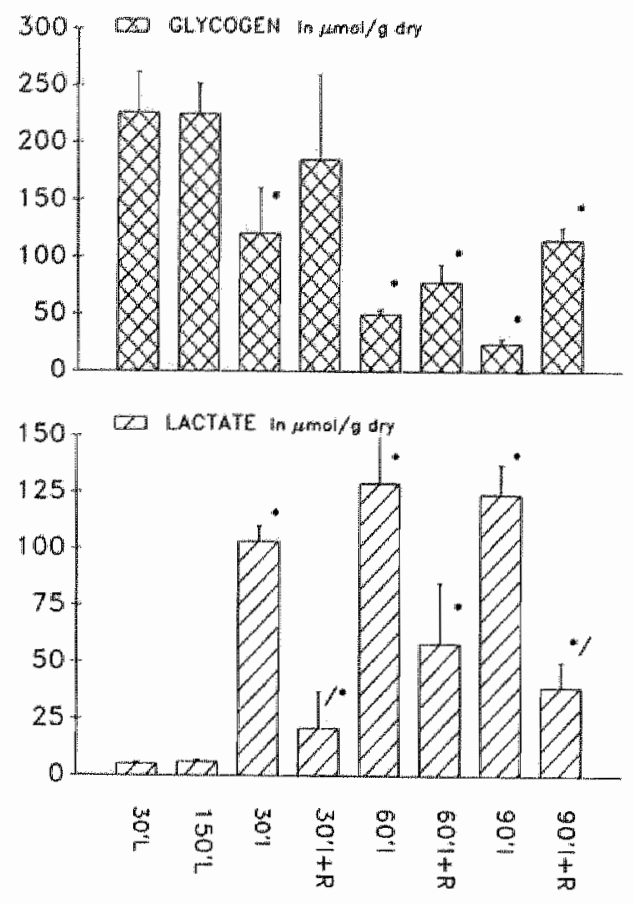

Figure 5.3. Bar diagrams showing the rissue levels of glycogen (a) and lactore (b). expressed as means and SEM per experimenal group.

(a) The tissue conten of givcogen gradually decreases winh prolonged duration of ischemia, while during reperfusion the level of glycogen lends 10 be restored lowards the control level. * indicates a significons $\quad(P<0.05)$ difference with the control perfissed groups fexcept for the 30 't group; only with the $150 \mathrm{~L}$ control group and for the $907+R$ group; with boith conrol groups and the 901 groupl. using the MarnWhithey U-rest (upper graph).

(b) The visswe content of lactale drastically increases after 30 minutes of ischemia. while reperficsion decreases the

level of lactare rowards the control level. * indicates a significant $(P<0.05)$ difference with the control perfised groups (except for the 601 and $601+R$ groups: only with the $302 \mathrm{~L}$ control group), $1^{*}$ indicates a significant $(P<0.05)$ difference with the 301 group and * indicates a significan

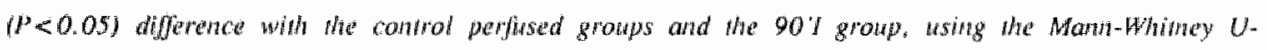
test Hower graphy.

ischemia, as compared with 90 minutes of ischemia without reperfusion, indicating restoration of the tissue content of glycogen (Fig. 5.3a).

The tissue content of lactate, roughly $5 \mu \mathrm{mol} / \mathrm{g}$ dry weight in control hearts, was significantly increased in all hearts rendered ischemic. Reperfusion tended to reduce tissue levels of lactate. Although the tissue content of lactate was significantly lower in reperfused hearts than in hearts rendered ischemic for the same period but 
without reperfusion, it remained significantly higher than in control hearts (Fig. 5.3b).

\subsubsection{Correlations between multilamellar vesicles and metabolic alterations.}

The increase in the number of multilamellar vesicles was most pronounced when the duration of ischemia was 60 minutes or longer (MLV-T-L, MLV-MITO) or when hearts were reperfused after ischemic periods of minimal 60 minutes (MLV-SL). These changes correlated in time with the accumulation of fatty acids in general and arachidonic acid in particular, and with the decrease in tissue content of ATP. The strength of a possible relation between multillamellar vesicles and metabolic parameters was expressed as the coefficient $\left(R_{s}\right)$ of rank correlation (Table 5.3).

\subsection{DISCUSSION}

A striking feature, observed in the isolated ischemic and reperfused rabbit myocardium, is the increase in multilamellar vesicles extruded from sarcolemma and mitochondrial membranes following these interventions. Although there is some doubt about the shape, occurrence and number of these multilamellar vesicles in vivo, it has been argued that they can be used to quantitate ischemia induced membrane destabilization (194). Multilamellar vesicles have been observed in rabbit hearts with freezefracturing, without the use of tannic acid (164). However. it cannot be excluded that tannic acid fixation has an additional effect on the formation of multilamellar vesicles (193, 194). Whether multilamellar extrusions are formed either directly due to biochemical or physico-chemical induced changes in the membrane architecture or indirectly under the influence of tannic acid fixation is not known, but it is generally accepted that the formation of these extrusions reflects membrane damage.

In the present study we observed a relation in time between the increase in the number of multilamellar vesicles and metabolic alterations, such as accumulation of fatty acids in general and arachiclonic acid in particular, and a decrease in the tissue content of ATP. In addition, the LDH release during reperfusion shows a strong correlation with the number of multilamellar vesicles extruded from the sarcolemma 
TABLE 5.2. Biochemical parameters in rabbir thearts under various experimentall conditions.

\begin{tabular}{|c|c|c|c|c|}
\hline & n & $\frac{\text { P LIPID }}{(\mu \mathrm{mol} / \mathrm{g} \mathrm{dry})}$ & $\frac{\mathrm{TG}}{(\mu \mathrm{mol} / \mathrm{g} d \mathrm{dy})}$ & $\frac{\mathrm{FA}}{(\mathrm{nmol} / \mathrm{g} d r y)}$ \\
\hline $30^{\prime} \mathrm{L}$ & 5 & $218 \pm 9$ & $92 \pm 26$ & $418 \pm 38$ \\
\hline $150^{\prime} \mathrm{L}$ & 3 & $193 \pm 12$ & $82 \pm 28$ & $443 \pm 122$ \\
\hline $30^{\prime} \mathrm{L}+30^{\prime} \mathrm{I}$ & 4. & $205 \pm 6$ & $72 \pm 11$ & $751 \pm 252$ \\
\hline $30^{\prime} \mathrm{L}+30^{\prime} \mathrm{I}+30^{\prime} \mathrm{R}$ & 3 & $199 \pm 12$ & $103 \pm 29$ & $1053^{*} \pm 339$ \\
\hline $30^{\prime} \mathrm{L}+60^{\prime \prime} \mathrm{I}$ & 3 & $236 \pm 12$ & $107 \pm 21$ & $1528^{\text {雨 }} \pm 567$ \\
\hline $30^{\prime} \mathrm{L}+60^{\circ} \mathrm{I}+30^{\prime} \mathrm{R}$ & 3 & $239 \pm 29$ & $206 \pm 116$ & $1990^{\circ /} \pm 443$ \\
\hline $30^{\prime} \mathbf{L}+90^{\prime} \mathrm{I}$ & 4 & $195 \pm 7$ & $81 \pm 28$ & $1146^{*}=112$ \\
\hline $30^{\prime} L+90^{\prime} I+30^{\circ} \mathrm{R}$ & 5 & $212 \pm 11$ & $186 \pm 42$ & $1525^{*} \pm 356$ \\
\hline
\end{tabular}

The data are presented as mean values per experimental group and standard errors of the mean (SEM), whereas $n$ is the number of rabbits examined per group. Tissue contents are expressed in $\mu m o l / g$ dry weight for P-LIPID (phospholipids) and TG (triacylglycerols) and in $\mathrm{nmol} / \mathrm{g}$ dry weight for FA (fatty acids). L. I and R refer to Langendorff perfusion, Ischentia and Reperfusion (Langendorff), respectively. * indicates a significant $(\mathrm{P}<0.05)$ difference with the control perfused groups, using the Mann-Whitney U-test.

$\left(R_{1}=0.972\right.$ and $\left.R_{s}=1.000\right)$. Although based on a limited number of experiments, this again suggests a relation between the extrusion of multilamellar vesicles and permeability changes of the sarcolemma.

The tissue levels of lactate and glycogen have been determined to indicate the severity of the ischemic insult. It is interesting to note that the tissue content of lactate had already drastically increased after ischemic periods as short as 30 minutes. when no significant increase in the number of multilamellar vesicles could be observed. Both the tissue levels of lactate and glycogen tended to be restored to control levels upon reperfusion. Whether the decrease in the tissue level of lactate is merely the effect of washout due to reperfusion, or that active excretion and/or passive diffusion out of the cell due to reperfusion induced dysfunction of the sarcolemma also plays a role remains unclear.

Regarding the time-course relation between alterations in the lipid-metabolism and multilamellar vesicles, Van Bilsen and colleagues (234) noted that the accumulation 
TABLE 5.3. Spearman's coefficient of rank correlation $\left(R_{s}\right)$ between the mean values of multilamellar vesicles and severai biochemical parameters, examined per experinental group.

\begin{tabular}{|c|c|c|c|}
\hline & $\frac{M L V-S L}{\left(R_{s}\right)}$ & $\frac{\text { MLV-MITO }}{\left(\mathrm{R}_{\mathrm{s}}\right)}$ & $\frac{M L V-T-L}{\left(R_{S}\right)}$ \\
\hline FA & $0.79^{*}$ & $0.81^{*}$ & 0.90 \\
\hline $\mathrm{AA}$ & $0.71^{*}$ & $0.79^{*}$ & $0.86^{\circ}$ \\
\hline P-LIPID & $0.26_{*}$ & 0.69 & 0.48 \\
\hline ATP & $-0.74^{*}$ & $.0 .68^{\circ}$ & $-0.79^{\circ}$ \\
\hline
\end{tabular}

* and ** indicate significant relation between the two variables at $P<0.05$ and $\mathrm{P}<0.01$, respectively. n.d. means that linear regression was not determined.

MLV-SL, MLV-MITO and MLV-T-L are nultilamellar vesicles extruded from sarcolemna or mitochondrial membrane and the total number of multilamellsr vesicles observed, without the multilamellar vesicles extruded from or adjacent to lipid droplets, respectiwely.

FA, AA, P-LIPID and ATP refer to fatty acids, arachidonic acid, phospholipidls and adenosine triphosphate, respectively.

of fatty acids in ischemic rat hearts started after 45 to 60 minutes, a time interval in which the transition from reversible to imeversible damage is likely to take place (116, 184). A similar time interval was observed in the present study performed on isolated rabbit hearts.

A variety of mechanistic explanations has been given for membrane destabillization during myocardial ischemia. Firstly, the depletion of ATP below a critical level may lead to membrane disorders and disturbed interaction between membranes and the cytoskeleton (116, 121, 179). Secondly, phospholipid degradation may occur, due to enhanced enzymatic hydrolysis by phospholipases $(16,87,254,260)$ or impaired resynthesis of phospholipids $(44,234)$, Thirdly, changes in the ionic $\left(\mathrm{H}^{+}, \mathrm{Ca}^{2+}\right)$ environment $(148,200)$ may alter the physico-chemical characteristics of the phospholipids in the bilayer and render them more susceptible to phospholipase altacks $(42$, 72). Fourthly, the intercalation of amphipathic compounds may have similar effects on the sarcolemma. eventually leading to functional and structural impaiment. These mechanistic explanations of membrane destabilization may be directly or indirectly involved in the formation of multilamellar extrusions.

During reperfusion oxygen free radicals can be formed causing further deterioration of the sarcolemma, as may be caused by peroxidation of fatty-acyl chains of the 
phospholipids, and hampering it to act as a permeability barrier. This may add to osmotic load increases $(42,116,259)$, associated with a massive influx of $\mathrm{Ca}^{2+}(200$, 201), on its tum leading to enhanced activation of proteases and phospholipases (72, 254). Consequently, reperfusion may add to enhanced impaiment rather than to recovery.

Our experiments clearly show a significant depletion of ATP and accumulation of fatty acids (nmolar range) of endogenous origin, for example, from phospholipids or triacylglycerols. The latter source is probably less likely, because no significant alterations were obsenved in the tissue content of triacylglycerols and in the number or size of lipid droplets (data not shown), indicating that lipolytic activity is not enhanced under these circumstances. Exogenous sources of fatty acids can be excluded since no fatty acid or triacylglycerols, from which fatty acids can be released by action of endothelial lipoprotein lipases (220), were added to the perfusion medium.

A causal relation between the appearance of multilamellar vesicles and alterations in lipid metabolism is a possibility to be considered. The present data indicate that multilamellar vesicles appear in cardiac tissue, the ATP levels of which were decreased

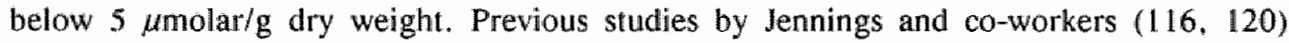
reveal that cell death first occur when the content of ATP is depleted to a comparable low level. From recent work of Van Bilsen and associates (234) it can be inferred that a similan relationship exists between ATP depletion and the accumulation of fatty acids, including arachidonic acid, in ischemic cardiac tissue. Impaired resynthesis of phospholipids. most likely caused by inhibition of acyl-CoA synthetase by AMP and adenosine, accumulating during ischemia, and the decrease of ATP, necessary as cosubstrate in the resynthesis of phospholipids (234) may directly lead to destabilization and dysfunction of membranes. These disturbances may lead to accumulation of lysophospholipids and fatty acids, potent inhibitors of membrane function and structure (50, 127). In this regard it is worthwhile to mention that although the number of multilamellar vesicles extruded from the sarcolemma is drastically increased in reperfused hearts after prolonged ischemia, this is not reflected in the biochemical parameters (such as the accumulation of fatty acid). This seems to be contradictory when a direct causal relation between the formation of multilamellar vesicles and the accumulation of amphipathic compounds is proposed. A possible explanation is that 
during the ischemic period the sarcolemma is already predisposed for changes to occur during reperfusion or that part of the fatty acids accumulated during ischemia are oxidized by mitochondrial activity during reperfusion, by virtue of restored supply of oxygen.

In conclusion the findings in the present study indicate that muitilamellar vesicles are an indication of the onset of irreversible cell damage. The determination of the number of multilamellar extrusions can be used as a tool to rate membrane injury and this may provide more insight into the pathogenesis of ischemia and reperfusion induced membrane injury.

\subsection{SUMMARY}

Alterations in lipid metabolism are important mediators of functional and structural membrane damage during myocardial ischemia. The time course of multilamellar vesicle formation and its relation to metabolic alterations were investigated in isolated rabbit hearts, rendered ischemic for 30,60 or 90 minutes, a subset of which was reperfused for 30 minutes. The increase in the number of multilamellar extrusions in cardiomyocyte membranes was most marked after ischemic periods of 60 minutes or longer. Reperfusion of ischemic tissue elicits a further increase in the number of multilamellar vesicles. In the same time interval a significant accumulation of fatty acids in general, arachidonic acid (a marker for phospholipid breakdown) in particular, and a significant decrease in the tissue content of ATP was observed. Lactate dehydrogenase ( $\mathrm{LDH}$ ) was released from the hearts during reperfusion. No significant alteration in the total tissue content of triacylglycerols and phospholipids was detected. The amount of fatty acids accumulated in the hearts only reflect the breakdown of a small fraction of phospholipids. These findings indicate that the degradation of a small proportion of phospholipids might have serious pathophysiological consequences, as indicated by the increase in the number of multilamellar vesicles and the release of LDH, when a causal relationship between morphological alterations and enhanced lipolysis or phospholipid breakdown does exist in the ischemic and reperfused rabbit heart. 

CHAPTER 6

MULTILAMELLAR EXTRUSIONS AND ALTERATIONS IN LIPID METABOLISM IN ISOLATED, ISCHEMIC AND REPERFUSED RAT HEARTS: A COMPARISON WITH RABBIT HEARTS 


\subsection{INTRODUCTION}

It is generally believed that myocardial sarcolemmal dysfunction plays an important role in the pathogenesis of ischemic myocardial cell injury $(106,129,259)$. In recent years, alterations in lipid metabolism have been considered to be involved in ischemic membrane dysfunction $(50,127,234,243)$. Alterations in the content and composition of membrane phospholipids and the generation of amphipathic metabolites during ischemia may directly affect the integrity of myocardial membranes and lead to functional and structural alterations.

Extrusion of multilamellar structures from myocardial sarcolemma and mitochondrial membranes is likely to be one of the earliest structural manifestations occurring during ischemia and reperfusion (189. 193, 250). These multilamellar extrusions, as observed in rabbit hearts, are probably a reflection of subtle membrane alterations caused by destabilization of the lipid bilayer (193-195, 250).

The formation of multilamellar extrusions from cardiac membranes during tannic acid based fixation might well depend on the phospholipid composition of the membranes, as indicated by the findings in model experiments (195). It was found that the induction of aggregation and the formation of multilamellar vesicles differed considerably, when comparing rat or rabbit heart phospholipid extracts. Since the described interaction of tannic acid with phospholipids was found to be restricted to choline headgroup containing phospholipids and the relative composition of the plasmalogen phosphatidalcholine and sphingomyelin (195) differed between these species. it was concluded that these differences in composition might be responsible for the differences observed between rabbit and rat heart extracts. Preliminary experiments in our laboratory also indicated that in isolated ischemic and reperfused hearts differences between rat and rabbit had to be considered. as far as the formation of multilamellar vesicles is concerned.

Therefore, it was the aim of this study to investigate whether the formation of multilamellar extrusions is a more general feature of membrane damage or limited to one species. To this end the number of multilamellar vesicles extruded from sarcolemma, mitochondria and lipid droplets was measured in ischemic and reperfused rat hearts. The data obtained in these experiments were compared with those previously 
obtained in rabbit hearts. It was investigated whether the formation of multilamellar vesicles in rats correlates with a semi-quantitative scoring method of ischemic cell injury (185) and the biochemical changes with emphasis on lipid metabolism, in a similar way as in the rabbit experiments.

\subsection{MATERIAL AND METHODS}

\subsubsection{Preparation of the hearts.}

The experiments were performed on male Lewis rats, weighing between 250 and $325 \mathrm{~g}$, mildly anesthetized with diethylether. After thoracotomy the hearts were excised and immediately immersed in ice-chilled Tyrode's solution. The hearts were weighed after remnant lung tissue and fat had been removed. The hearts were then attached to the aortic cannula of an earlier described perfusion system (210).

\subsubsection{Perfusion conditions,}

The hearts were retrogradely perfused for the first 10 minutes as described in more detail in chapter 4.2.2. After the initial 10 minutes period of retrograde perfusion $\left(10^{\circ} \mathrm{L}\right)$ hearts were subjected to antegrade perfusion with the same medium. A catheter connected to a pressure transducer (CTC) was inserted into the left ventricle through the apex to measure left ventricular pressure.

\subsubsection{Experimental conditions.}

After the first 10 minutes of retrograde perfusion $\left(10^{\circ} \mathrm{L}\right) 4$ hearts were ante. gradely perfused for 20 minutes. which allows the heart to eject fluid through the aorta $\left(20^{\circ} \mathrm{W}\right)$. These 50 -called "working hearts" were considered to be the control hearts $\left(10^{\circ} \mathrm{L}+20^{\circ} \mathrm{W}\right)$.

In a separate series of experiments 4 hearts were subjected to 90 minules of global. no-flow ischemia (I) following the initial control perfusion period, as described 
above $\left(10^{\prime} \mathrm{L}+20^{\prime} \mathrm{W}+90^{\prime} \mathrm{I}\right)$. Ischemia was accomplished by complete occlusion of the cannula connected with the left atrium and the aorta.

In another series of experiments the ischemic period was followed by retrograde reperfusion of 10 minutes and subsequently by a 20 minutes antegrade reperfusion $(R)$, under the same conditions as during the initial control period $\left(10^{\prime} \mathrm{L}+20^{\prime} \mathrm{W}+90^{\prime} \mathrm{I}+30^{\prime} \mathrm{R}\right.$. $n=4)$.

The temperature of the hearts was monitored with thermoprobes throughout the experimental period and kept at $37 \pm 1^{\circ} \mathrm{C}$.

\subsubsection{Fixation and tissue processing.}

At the end of the experiments biopsies were taken for biochemical analysis, in a way described in Chapter 5.2.2. Thereafter the remainder of the hearts were fixed by perfusion (at a perfusion pressure of $8 \mathrm{kPa}$, at $25^{\circ} \mathrm{C}$ ) with a combination of $2.5 \%$ glutaraldehyde and $2 \%$ tannic acid in $20 \mathrm{mM}$ phosphate buffered physiological saline (PBS, pH 7.2) for 10 minutes. Midwall biopsies (about $1 \mathrm{~mm}^{3}$ ) were cut from the left ventricle and further fixed by immersion at room temperature for 2 hours in the same fixative.

After fixation the tissue pieces were further processed for electron microscopic examination, as previously described in Chapter 4.2.4.

\subsubsection{Morphometric analysis.}

For each experiment 10 micrographs were randomly taken from one grid at the same magnification. Micrographs were analyzed morphometrically (Kontron; MOPvideoplan) to establish the number, size and (sub-ycellular location of multilamellar vesicles, as described in more detail in Chapter 4.2.5.

The degree of ischemic injury was evaluated on electron micrographs (10-15) according to a slightly modified semi-quantitative scoring system, as described by Schaper et al. (185). Evaluation of the ultrastructural appearance (4 hearts per experimental group) was performed as described in more detail in Chapter 4.2.5. 
6.2.6. Analysis of tissue lipids.

The freeze-clamped tissue biopsies were processed for analysis of tissue lipids as described in detail in Chapter 5.2.5.

\subsubsection{Statistical analysis.}

All data are presented per experimental group as mean values and standard error of the mean (SEM). Differences between groups were analyzed for statistical signifi. cance using the Mann-Whitney U-test. P-values less than 0.05 were considered to be statistically significant.

Spearman's coefficient of rank correlation $\left(R_{S}\right)$ was used to estimate the strength of the relation between two variables. Correlations between the morphological and biochemical parameters (in rat hearts) were considered per experiment as data from individual hearts and not as the means per experimental group, as was previously done for comparable experiments with rabbit hearts. This approach was chosen because in the rat experiments only three experimental groups were investigated, which is inadequate to obtain statistical significance. The association between two parameters obtained with the Spearman rank correlation were tested for statistical significance $(\mathrm{P}<0.01)$.

The data (as means per experimental group) obtained in the rat experiments were

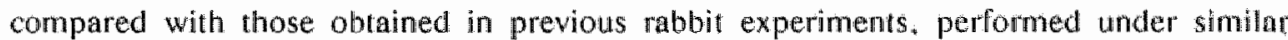
experimental conditions $(30$ minutes retrograde perfusion, 90 minutes of global ischemia and 90 minutes of ischemia followed by 30 minutes retrograde reperfusion for the control and the experimental groups, respectively, for a more detailed description see Chapter 4.2.3). Differences between the data per experimental group in rats and in rabbits were analyzed for statistical significance $(P<0.05)$ using the Mann-Whitney $U$ test. 


\subsection{RESULTS}

\subsubsection{Morphological alterations.}

\subsubsection{General morphologic appearance.}

After control perfusion $\left(10^{\circ} \mathrm{L}+20^{\circ} \mathrm{W}\right)$ the rat hearts examined had a normal appearance: myocytes had normal mitochondria (without matrix granulae) and nuclei. and normal to slightly contracted sarcomeres. No intracellular or extracellular edema could be observed and glycogen appeared to be present in normal quantities.

Rat hearts subjected to severe ischemia ( $90 \%)$ displayed moderately to severely damaged myocytes. Generally, the mitochondria had moderately or abundantly cleared matrices, with broken cristae and sometimes flocculent densities. Most of the nuclei had a light density of the chromatin, which was pyknotic. In general, the sarcomeres were partially relaxated. No extracellular edema was observed, but (moderate) intracellular edema was present in some rat hearts. All cardiomyocytes examined were lacking glycogen.

Rat hearts subjected to severe ischemia and reperfusion $\left(90^{\prime} I+30^{\prime} R\right)$ generally contained severely damaged myocytes. The mitochondria had abundantly cleared matrices with broken cristae and generally flocculent densities. Practically all nuclei had light density of the chromatin and were severely pyknotic. The sarcomeres were sometimes contracted, but no hypercontraction was observed. Moderate extracellular edema was observed in almost all rat hearts, whereas in some hearts intracellular edema was more abundantly present. No glycogen was observed in the cardiomyocytes examined.

\subsubsection{1schemic injury scoring.}

The degree of myocardial ischemic injury, as scored on a semi-quantitative scale, was found to be significantly increased in rat hearts subjected to 90 minutes of ischemia. Reperfusion of ischemic hearts tended to lead to a further increase in the ischernic injury score (Fig. 6.1a). When these data, as obtained in rat hearts, are compared with corresponding data obtained in rabbit hearts, a similar pattern was 
observed. The rat hearts tended to be slightly more affected by reperfusion than rabbit hearts (Fig. 6. Ia).

\subsubsection{Multilamellar vesicles.}

In cardiomyocytes of ischemic and/or reperfused rat hearts multilamellar vesicles were found extruded from the sarcolemma, mitochondria and in or adjacent to lipid droplets. Only moderate differences in the number of multilamellar extrusions were observed, as compared with the previous rabbit experiments under similar experimental conditions. The differences were limited to the number of multillamellar vesicles in or adjacent to lipid droplets (MLV-LIPID, Fig. 6.1e) and the total number of observed muitilamellar extrusions (MLV-TOTAL, Fig. 6.1b) in both ischemic and reperfused hearts and in the number of multilamellar extrusions from mitochondria (MLV-MITO, Fig. 6.1d) after 90 minutes of ischemia with reperfusion.

However, a significantly smaller number of multilamellar extrusions was found in rat hearts than in rabbit hearts when the individual scorings of (MLV-TOTAL) per heart were considered as one group of data (and not per experimental group).

In general the relative distribution of the observed multilamellar vesicles was comparable to that in rabbit hearts under similar experimental conditions. The percentages of observed multilamellar vesicles was $31 \pm 7 \%, 29 \pm 9 \%$ and $41 \pm 7 \%$ (as mean percentages \pm SEM) extruded from the sarcolemma, mitochondria or lipid droplets, respectively, in rat hearts and $28 \pm 6 \%, 27 \pm 7 \%$ and $43 \pm 7 \%$, respectively, in rabbit hearts.

The size of multilamellar extrusions (mean area $\left(\times 10^{-3}\right)$ of MLV-TOTAL \pm SEM) was $32 \pm 4 \mu \mathrm{m}^{2}$ in rat hearts and $48 \pm 5 \mu \mathrm{m}^{2}$ in rabbit hearts, a signilicant difference of approximately $33 \%$.

In rat hearts the mean number of (MLV-TOTAL) was significantly increased after 90 minutes of ischemia, whereas reperfusion did not lead to a further increase (Fig. 6.1b). A similar pattern was observed for rabbit hearts.

The number of (MLV-SL) appeared to be hardly affected by 90 minules of ischemia, whereas a drastic (more than 3-fold) and significant increase was observed when the rat hearts were subjected to reperfusion after 90 minutes of ischemia (Fig. 6.1c). An almost identical pattern was observed for rabbit hearts under similar experimental conditions. 

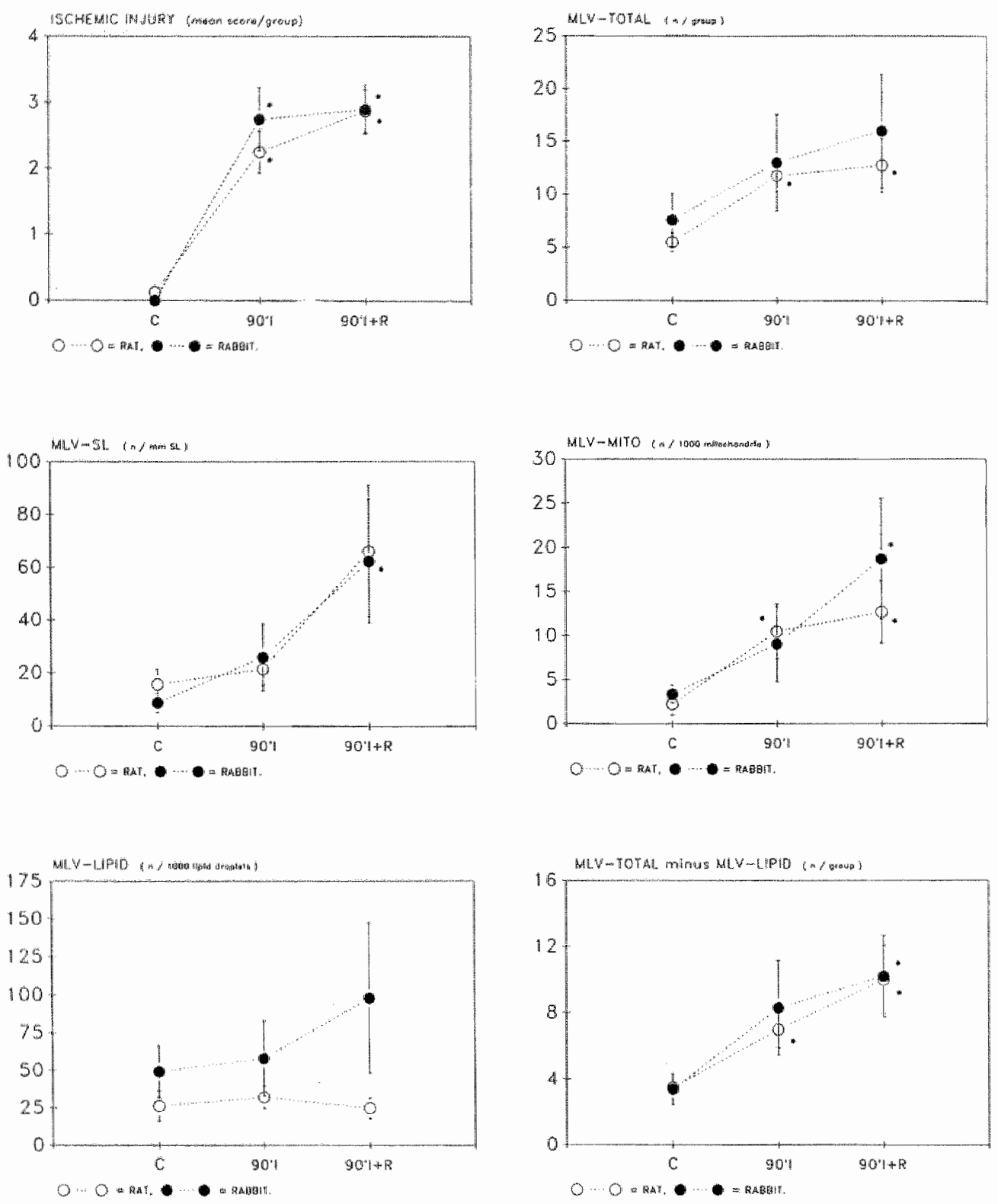


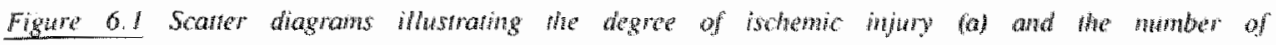

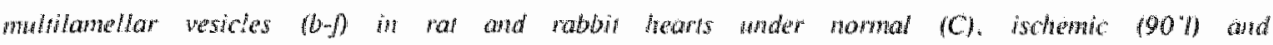
nepeysured $(901+R)$ condinoms.

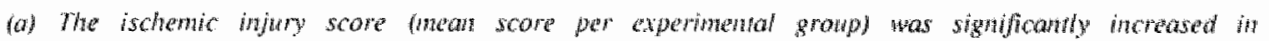
ischernic and in reperfinsed heorts in bont ras and rabbirs.

(b) The sum of observed mullilameliar vesicles in total (ML W-TOTAL, mean number obsemed pep"

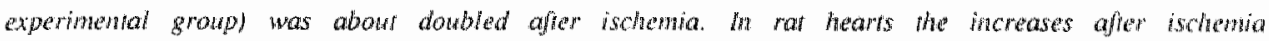
and reperfusion were found to be statisticaliy signfican.

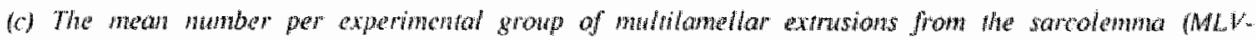

SL, expressed as nhm sarcolemmal was drasticalty increased in both rat and rabbit hears after reperfusion. This increase was found to be statisticaly signifrom in mabils.

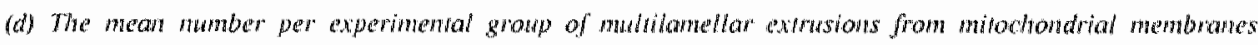

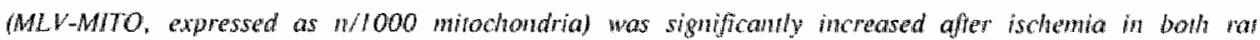

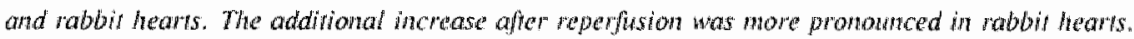

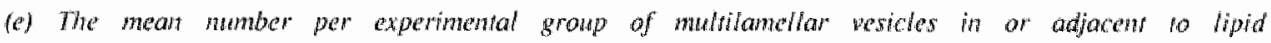

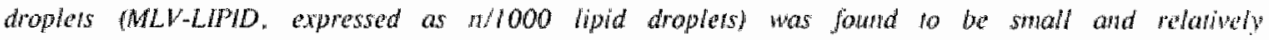

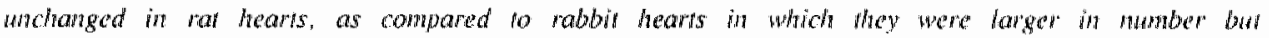
exhibived a greater variation.

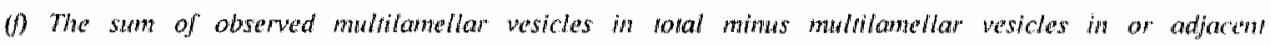
to fipid droplers (MLV-TOTAL minus MLV-LWTD, mean number observed per expertmenral growp) was" increased in borh rat and rabbit hearts affer ischemia and tended to increase further wpon

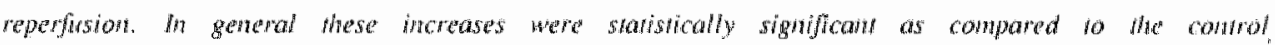

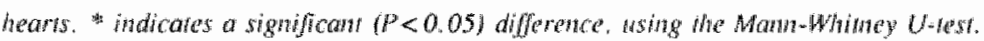

The number of (MLV-MUTO) was significantly increased after 90 minutes of ischemia, but was hardly affected by subsequent reperfusion (Fig. 6.1d). In rabbit hearts the effect of reperfusion on MLV-MITO seems to be more pronounced than in rat hearts.

The number of (MLV-LIPID) was unchanged under the various experimental conditions (Fig. 6. (e). The number of MLV-LIPID was higher in rabbit hearts than in rat hearts, although this difference is not statistically significant due to the variation in the rabbit hearts. 
The number of MLV-TOTAL without taking MLV-LIPID into consideration (MLVTOTAL minus MLV-LIPID), showed a significant increase after 90 minutes of ischemia. in both rat and rabbit hearts and tended to increase further upon reperfusion (Fig. 6. 11).

\subsubsection{Metabolic alterations.}

The tissue content of fally acids, approximately $240 \mathrm{nmol} / \mathrm{g}$ dry weight in control hearts, was drastically (almost 10 times) and significantly increased in rat hearts after 90 minutes of ischemia. Reperfusion seemed to have little effect and did not evoke a further increase, as observed in rabbit hearts (Fig. 6.2a). The accumulation of fatty acids was far more pronounced in rat hearts than in rabbit hearts. The amount of fatty acids was significantly lower in control rat hearts, while the accumulation in ischemic and in reperfused hearts was significantly greater in rats than in rabbits.

The tissue level of arachidonic acid, a fatty acid almost exclusively present in phospholipids, was approximately $19 \mathrm{nmol} / \mathrm{g}$ dry weight in control hearts. A drastic (about 10-fold) and significant increase was observed in rat hearts after 90 minutes of ischemia, whereas upon reperfusion the tissue level of arachidonic acid seemed to remain at that level, as compared with prolonged ischemia without reperfusion (Fig. 6.2b). In rabbit hearts a comparable pattern was observed, but the level of arachidonic acid was significantly lower than in ischemic and ischemic plus reperfused rat hearts.

The amount of triacylglycerols, about $16 \mu \mathrm{mol} / \mathrm{g}$ dry weight in control hearts, was found to be unchanged in rat hearts under the different experimental conditions. However, in rat hearts after 90 minutes of ischemia the triacylglycerol level was found to be about $22 \mu \mathrm{mol} / \mathrm{g}$ dry weight, a moderate but significant increase. This increase was not altered by reperfusion (Fig. 6.2c). In rabbits an inconsistent pattern was observed. After 90 minutes of ischemia the amount of triacylglycerols tended to decrease. After reperfusion the amount of triacylglycerols was significantly increased. as compared with the ischemic group. but this increase was not significant when compared with the control group. In general, the amount of triacylglycerols was significantly lower in rat hearts than in rabbit hearts. 
The amount of diglycerides. about $450 \mathrm{mmol} / \mathrm{g}$ dry weight in control hearts. tended to increase in rat hearts after 90 minutes of ischemia and to further increase upon reperfusion. After reperfusion the increase was significant, as compared with the ischemic group, but not as compared with the control group (Fig. 6.2d). In rabbit hearts only in some cases the amount of diglycerides was estimated. A similar pattern was observed in this species, but no significance levels were determined because of the limited number of experiments.

The amount of the total phospholipid pool. approximately $240 \mu$ mol fatty acyl moieties/g dry weight in the control situation. did not change consistently in both rat and rabbit hearts under the different experimental conditions. A slight, but significant increase (about $8 \%$ ) was observed in rat hearts after 90 minutes of ischemia (Fig. 6.2e). The amount of the total phospholipid pool was significantly greater in ischemic and reperfused rat hearts than in rabbit hearts.

6.3.3. Relation between the number of multilamellar vesicles and alterations in (lipid) metabolism.

The strength of a possible relation between the number of multilamellar extrusions and the metabolic parameters was expressed as the coefficient of the Spearman rank correlation and the coefficient of determination of the first order regression (Table 6.1). Only poor to moderate correlations were found between the ischemic injury score and multilamellar vesicles on the one hand, and diglyceride and phospholipid content on the other (data not shown). Poor correlations were also found when MLV-SL or MLV-LIPID were compared with the ischemic injury score or the tissue levels of fatty acids. arachidonic acid and triacylglycerols (Table 6.1). Moderately significant $(0.05<\mathrm{P}<0.01)$ correlations were found for the relation between MLVMITO and the tissue levels of fatty acids, arachidonic acid or triacylglycerols, whereas at significant correlation $(\mathrm{P}<0.01)$ was found between MLV-MITO and the degree of ischemic injury (Table 6.1). Ischemic injury score and MLV-TOTAL (not shown) and MLV-TOTAL minus MLV-LIPID on the one hand, showed very good correlations with the tissue levels of fatty acids. arachidonic acid or triacylglycerols (Table 6.1) on the other. 

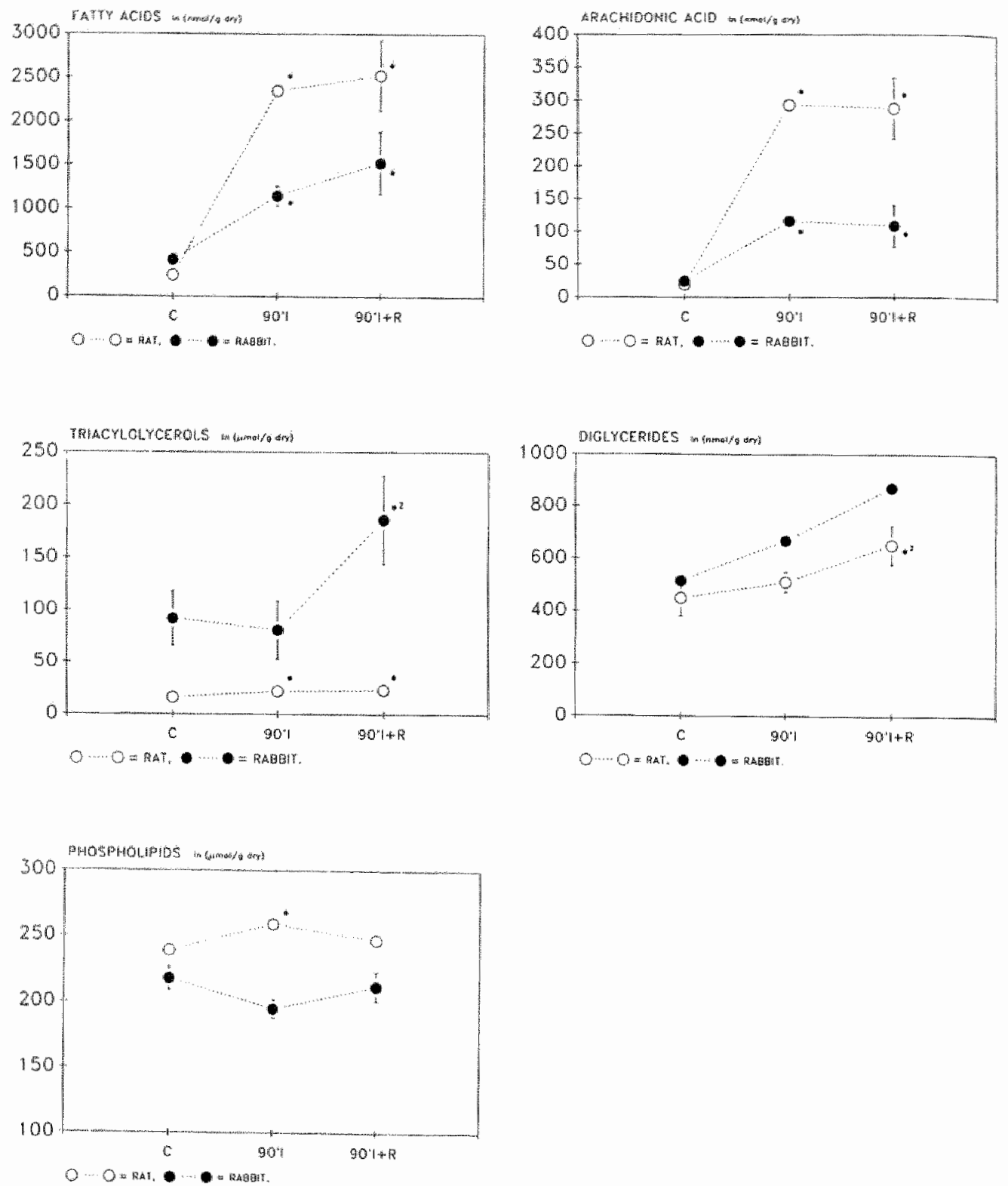


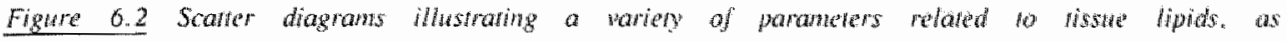
measured in rat and rabbit hears under control $(C)$, ischemic $(90 \%)$ and reperfivsed $(90 \%+k)$ conditions.

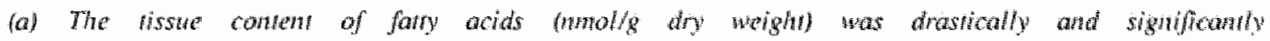

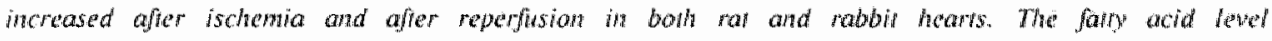
was significantly $(P<0.01)$ smaller in conmol ras and significanly $(P<0.0$ n) higher in ischemb and in reperfised rat hearts, as compared to rabbits.

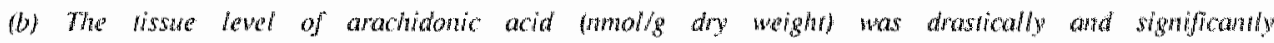
increased affer ischemia and after reperfusion in both wa and rabbir hearts. the arachidonic acti level was significantly $(P<0.01)$ higher in rat thon in rabbit hearts afher both ischtemia ant reperfusion.

(c) The tissue content of rracylglycerols (umalig dry weighi) was found to be moderately. but

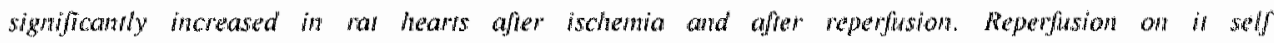
did not lead to a finther increase, as compared to the ischemic group. In rabbits, however. reperfusion caused a significan increase in the anoun of miacylglycerols as compared to the ischemic group, bu nat as compared to the control group. The riacyglycerol levels were significantly $(P<0.01)$ smaller in rat when in rabbit hearts.

(d) The announ of diglycerides (nmol/g dry neight) was similar in boh rat and rabbir hearss and

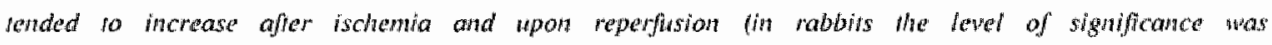
nos determinied).

(c) The lotal phospholipid pool (Amol/g dry weight) was significantly $(P<0.01)$ greater in rat than in rabbir hears affer ischemia and affer reperfiston. No nef decrease was found in elther of the cxperimental groups investigared.

All dara are presented as means per experimental group and 5 handard erpors of We mean (SEM). "* indicates a significan $(P<0.05)$ difference with the control perfused group (C) and the indicates a

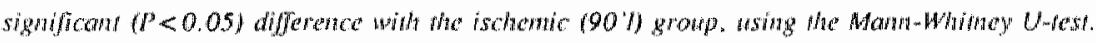

\subsection{DISCUSSION}

The mean size and the number of multilamellar vesicles are significantly smaller in rat than in rabbit hearts after 90 minutes of ischemia, with or without subsequent 
reperfusion for 30 minutes.

Despite the fact that correllations between the different parameters could not be performed per experimental group (to avoid possible sampling errors leading to a greater variation in the data) and had to be estimated from the individual data, the findings in this study indicate that in rat the number of multilamellar extrusions (MLV-TOTAL and MLV-TOTAL minus MLV-LIPID in particular, and MLV-MITO to a lesser degree) correlates positively with the ischernic injury score as well as with the accumulation of fatty acids, including arachidonic acid and triacylglycerols. It has to be emphasized that the number of multilamellar vesicles extruded from the sarcolemma (MLV-SL) is particularly increased during reperfusion. In general these findings are similar to those previously obtained in rabbit experiments.

It has been suggested that the accumulation of fatty acids, which are amphipathic compounds, may directly affect the integrity of the membrane lipid bilayer ( 50 . 127). Therefore. the possibility that the accumulation of these compounds is causitively related to the formation of multilamellar extrusion has to be considered. However, as compared with the rabbit experiments, a significant greater accumulation of fatty acids in general, and of arachidonic acid in particular, is observed in rats. Besides. the size and the number of multilamellar extrusions were found to be smaller in the hearts. These observations seem to be contradictory to a causative relation. One should keep in mind, however, that the difference in the phospholipid composition of rabbit and rat hearts, as indicated in chapter 3, may contribute to the observed difference in the size and number of multilamellar extrusions. At the present state of the art it is difficult to draw a final conclusion.

The findings in the present study indicate that relatively small degradation of phospholipids may already lead to disorganization in the membranes to such an extent that formation of multilamellar vesicles occurs during tannic acid based fixation. In the previously performed experiments in rabbits the formation of multilamellar extrusions was found to be associated with an increased permeability of the sarcolemma, as suggested by a significant leakage of cellular enzymes. The present findings also support the idea that relatively subtle alterations in the content and composition of membrane phospholipids are involved in the onset of irreversible myocardial ischemic injury $(140,180)$, rather than disruption of the sarcolemma $(88,116,117)$. 
TABLE 6.1. Spearman's coefficient of rank correlation $\left(\mathbb{R}_{s}\right)$ between (the individual walues per rat hearts of multilamellar extrusions, ischemic injury score and severat parameters related to tissue lipid content.

\begin{tabular}{|c|c|c|c|c|}
\hline & $\frac{\mathrm{II}}{\left(\mathrm{R}_{\mathrm{S}}\right)}$ & $\frac{F A}{\left(\mathbb{R}_{8}\right)}$ & $\frac{A^{A}}{\left(R_{s}\right)}$ & $\frac{\mathrm{TG}}{\left.(\mathbb{R})_{\mathrm{s}}\right)}$ \\
\hline II & $\operatorname{xxxx}$ & $0.87^{328}$ & $0.82^{404 k}$ & 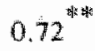 \\
\hline$M \mathbb{L V}-\mathrm{SL}$ & $0.30_{\text {隶事 }}$ & $0.29 *$ & $0.06{ }_{*}$ & $0.43_{\mathrm{ws}}$ \\
\hline MLV-MITO & $0.81^{1.4}$ & $0.63^{*}$ & $0.66^{*}$ & $0.70^{6 / 2}$ \\
\hline MLV-LIPID & -0.08 & $0.077_{*, 0 k}$ & -0.10 & $0.05_{\text {䍀取 }}$ \\
\hline MLV-T-L & 0.94 & $0.87^{\text {pip }}$ & $0.80^{n-m}$ & $0.79^{\circ}$ \\
\hline
\end{tabular}

* and ** Indicate a significant relation between the two variables at $P<0.05$ and $P<0.01$. respectively. II is ischemic injury score and MLV-SL, MLV-MITO. MLV-LIPID are multilamellar vesicles extruded from sarcolemma, mitochondria or lipid droplets, respectively. MLV-T-I. is the number of multilamellar vesicles, as observed in total, without the multilamellat vesicles extruded from lipid droplets. FA, AA and TO refer to fatry acids, arachidonic acid and triacylglycerols, respectively.

However, the possibility of transient leakage has also to be considered, since LDH is a relative large molecule.

More research is needed to investigate possible mechanisms involved in the formation of multilamellar extrusions. Knowledge of the exact phospholipid composition of myocardial membranes (166) and the interaction of membrane lipids with cytoskeleton proteins on the one hand and the glycocalyx on the other, under both normal and ischemic conditions, could shed more light on the possible mechanisms involved in membrane destabilization. The involvement of phospholipases has to be considered, since phospholipase activity may also be influenced by the phospholipid composition of membranes.

It is concluded that the development of multilamellar extrusions from membranes is not an unique feature for one species. but likely a more general phenomenon with similar mechanisms reflecting membrane damage as indicated by functional and biochemical criteria such as phospholipid degradation and membrane permeability. 


\subsection{SUMMARY}

It was investigated whether the formation of multilamellar extrusions in ischemic and reperfused hearts is restricted to one animal species or may be considered to be a more general phenomenon of membrane damage. To this end multilamellar vesicles were quantitated in rat hearts after control perfusion, 90 minutes of ischemia or after 90 minutes of ischemia followed by reperfusion. In the same hearts the degree of ischemic injury was scored on the basis of a semi-quantitative scale and several parameters related to lipid metabolism were determined. The data obtained in rat hearts were compared with those obtained in previous experiments on rabbit hearts.

It was found that the general distribution of multilamellar vesicles (extruded from sarcollemma, mitochondria and in or adjacent to lipid droplets) was similar in rat and rabbit hearts. The observed number of multilamellar vesicles and their size was found to be significantly smaller in rat than in rabbit hearts. The accumulation of fatty acids, in general, and arachidonic acid in particular was found to be significantly greater in rat than in rabbit hearts. Although no net decrease of phospholipids ( $\mu$ molar range) could be observed, the significant (in both rats and rabbits) accumulation of arachidonic acid (nmolar range), indicates that multilamellar extrusion may be a reflection of subtle, ischemic membrane alterations. 
CHAPTER 7

GENERAL DISCUSSION 
7.1. Membrame alterations and cell damage.

It is well accepted that alterations in membrarye structure and function play an important role in the pathogenesis of ischemia and/or reperfusion induced myocardial cell damage $(72,121,127)$. Until now the mechanisms involved in myocardial ischemic injury are incompletely understood. The cause seems to be multifactual because such mechanisms as acidosis, ATP depletion, accumulation of amphipathic compounds and phospholipid degradation likely contribute to the membrane disorders observed during myocardial ischemia and the onset of irreversible cell injury.

7.2. Tannic acid interaction(s) with phospholipids in a bilayered configuration."

Among the additives for lissue fixation, tamnic acid has been proposed to improve the preservation of lipid structures. such as the inclusion bodies in type 11 pneumocytes (125) and the multilamellar extrusions in ischemic and reperfused myocardium (164). Tannic acid does not only prevent extraction of saturated phosphalidylcholines (134, 178, this thesis chapter 2), but also induces the formation of multilamellar structures in both model system $(5,23$, this thesis chapter 2 and 3$)$ and biological material $(178,251)$.

The precise reaction mechanism underlying the interaction between tannic acid and phospholipids, specifically containing a choline headgroup, is incompletely understood. In our view tannic acid interacts (in an approximately one to one molar ratio) with the phospholipids in the bilayer arrangements, thereby probably altering the electrostatic or hydration repulsive forces between the lipid bilayers and, hence. facilitating aggregation and/or fusion. How this eventually leads to the tightly packed muliilamellar vesicles needs further investigation.

7.3. The use of tannic acid based fixation in ischemic and reperfused myocardium.

Based on the aforementioned findings we tend 10 consider the action of tannic acid based fixation to be a "cytochemical" demonstration of membrane destabilization. In the ischemic myocardium multilamellar vesicles. in our view likely to be formed 
during tannic acid based fixation, reflect stbtle ischemia induced membrane alterations. The accumulation of amphipathic lipids may contribute to destabilization of the myocardial membranes during ischemia, rendering phospholipids (particular those with a choline headgroup, lyso-phosphatidylcholine included) more susceptible to tannic acid interaction.

However, it has to be borne in mind that multilamellar vesicles have also been observed without the use of tannic acid, applying freeze-fracture electron microscopy (164). Unfortunately, in this situdy the multilamellar extrusions were not quantitated. so that it remains unclear whether these structures are, at least to some extent. additionally induced during tannic acid based fixation. It should be stressed that at the present state of knowledge these multilamellar vesicles are still to be considered as a reflection of ischemia and/or reperfusion induced membrane injury. irrespective of the fact whether they are formed "in viwo" or after tannic acid interaction with destabilized membranes.

How multilamellar vesicles might be formed "in vivo" is still incompletely understood. The accumulation of amphipathics is known to cause destabilization of membranes $(50,127)$. We have examined whether amphipathics might directly cause multilamellar vesicle formation. To this end arachidonic acid $(0.83 \mathrm{mM})$ was added 10 small unilamellar vesicles prepared from DMPC $(16 \mathrm{mM})$. In these experiments no aggregation or formation of multilamellar vesicles could be observed. This indicates that the accumulation of arachidonic acid, as observed in ischemic and reperfused myocardium. may only contribute indirectly to the destabilization of the sarcolemma. for instance, by making phospholipids more accessible to tannic acid.

It should also be kept in mind that the accumulation of $\mathrm{Ca}^{2+}$ and $\mathrm{H}^{+}$may affect membrane lipids by physico-chemical alterations inducing phase-transitions and plastseparations. which may lead to disturbances in the lipid domains and llie: "in vivo" formation of multilamellar extrusions (250). When such mechanisms exist, it implics that multilamellar extrusions may be formed without metabolic alterations of membrane lipids, such as the accumulation of amphipathics by phospholipase activity.

When multilamellar vesicles are indeed formed "in vivo" during ischemia and reperfusion it may have physiological consequences. Indeed, the formation of mulli. lamellar extrusions from membranes is likelly associated with increased permeability of 
membiranes, as indicated by the observed leakage of LDH in our experiments.

7.4. Species differences or similarities in the formation of multilamellar vesicles.

In both rat and rabbit experiments we have observed a similar relation in time between the increase in the number of multilamellar vesicles and metabolic alterations (accumulation of fatty acids, decreased level of ATP and leakage of LDH). In both species the number of multilamellar vesicles was independent of the experimental condition. These findings and the fact that multilamellar vesicles have also been observed in pigs (165) suggest that the formation of multilamellar extrusions is not restricted to one animal species, but can be considered to be a more general phenomenon of ischemic myocardial membrane injury.

However, it should be noted that the accumulation of fatty acids was far more marked in ischemic rat hearts compared to rabbit hearts, whereas this was not reflected by a comparable increase in the number of multilamellar membrane extrusions. We tend to explain this obvious contradiction by the difference in the content of phosholipids with a choline headgroup, which is smaller in rat than in rabbit hearts. Therefore, in rats less of these phospholipids might be affected by amphipathic accumulation and, hence, will be accessible to tannic acid.

7.5. Enzyme release (LDH) an indicator of membrane injury.

The release of lactate dehydrogenase (LDH), a cytosolic enzyme, into the coronary effluent may be considered as a sign of serious cell injury. However, in rats it has been shown that the amount of enzyme released is not related to functional recovery or the occurrence of ventricular fibrillation (233).

In our experiments in ischemic and reperfused rabbit hearts we have observed a relation in time between the cumblative release of LDH on the one hand and the increase in the number of multilamellar vesicles, the accumulation of fatty acids and the depletion of ATP on the other. At present we tend to believe that relative subtle membrane alterations, as might be reflected by the formation of multilamellar vesicles, may already lead to the leakage of LDH. However, because of the relative large size 
of LDH molecules, the possibility of transient leakage might also have to be appreciated.

Several studies have revealed that relatively subtle and partly reversible membrane alterations are involved in such a functional impairment of the sarcolemma (163). The findings suggest that the rupture of the sarcolemma (121) is not necessarily a prerequisite for the leakage of enzymes and macromolecules from the intracellular compartment.

7.6. ATP depletion, lipid changes and cell damage.

We have observed a rapid decrease in the tissue level of ATP, which appears to be related in time with the accumulation of fatty acids and the formation of multilamellar vesicles. There is a slight tendency that the ATP depletion precedes the accumulation of fatty acids, which could implicate that impaired resynthesis of phospholipids may indeed be involved in membrane destabilization (234). Besides, ATP depletion as substrate, resynthesis of phospholipids may also be hampered by blocking acyl-CoA transferase by AMP (234). Furthermore, a diminished level of ATP may influence the function of several membrane proteins and membrane-cytoskeleton interactions, since these are often regulated by phosphorylation $(35,218,231)$, in turn contributing to membrane alterations.

Lipid alterations, such as the accumulation of fatty acids and the degradation of a small proportion of cellular phospholipids ( $\mu$ molar range), as reflected be the accumulation of arachidonic acid (nmolar range) may lead to membrane destabilization. In our experiments we have observed a relation in time between these metabolic alterations on the one hand and the leakage of LDH and the increase of multilarnellar vesicles on the other. These findings seem to implicate that transient leakage or other relatively subtle membrane alterations, as may be reflected by the (direct or indirect) formation of multilamellar vesicles, can already cause significant permeability changes. 


\subsection{Glycocalyx alterations and cell damage.}

It is well accepted that membrane alterations play a significant role in the development of myocardial ischemic injury $(50,72,127,121,243)$. Altered membrane structure and function are frequently explained exclusively by biochemical and physico-chemical changes affecting membrane lipids. The possible interactions of membrane lipids with membrane proteins and of membrane constituents with the glycocalyx and the cytoskeleton elements need to be investigated.

The glycocalyx may play an important role in the regulation of ion transport (in particular of $\mathrm{Ca}^{2+}$ ) across the plasma membrane (138). Since calcium accumulation is thought to be involved in myocardial ischemic and/or reperfusion damage $(148,200)$. the influence of glycocalyx alterations has to be considered.

However " it has been documented that an intact glycocalyx adhering to the sarcolemma is not an indication of membrane integrity, since leakage of LDH could be observed in ischemic (45 minutes) and reperfused $(30$ minutes) rat hearts without a significant change in the ultrastructural appearance of the glycocalyx (188). It needs to be established whether at least to some extent "glycocalyx alterations may contribute to the loss of membrane integrity.

\subsection{Altered cytoskeleton interactions and cell damage.}

It has been demonstrated that the tissue level of ATP decreases during myocardial ischemia and reperfusion (116, 121,233, this thesis chapter 5 and 6). Since the interaction of cytoskeleton proteins with membrane components. as well as the structure and function of several membrane proteins, such as $\left(\mathrm{Na}^{+}, \mathrm{K}^{+}\right)$ATPase and $\mathrm{Ca}^{2+}$ ATPase, depend on phosphorylation $(35,218,231)$, they may well be affected during myocardial ischemia.

In isolated cardiomyocytes it has been demonstrated by the technique of photolysis, using fluorescently labeled lipid incorporated into the sarcolemma, that the lateral diffusion coefficient for such label probe increases by magnitude within 5 minules of hypoxia (74). It has been suggested that this increase in membrane fluidity is caused by removing the normal restrictions in the lateral diffusion in cardiac 
sarcolemma. The physical basis of these normal restrictions are supposed to reside in the linkage of the sarcolemma to its cytoskeletal scaffold.

Altered cytoskeleton interactions might lead to "blebbing" of the sarcolemma (see paragraph 1.2.3), as for example, microblebs are indicative of small breaks in the connections between the cytoskeleton and the sarcolemma $(91,163)$. Lesions in the cytoskeletal apparatus of myocardial cells are found to be correlated with increased osmotic fragility of irreversibly injured anoxic cardionyocytes $(162,163)$.

Immunofluorescence staining of vinculin, as observed over the entire plasma membrane, was found to be changed during total "in vitro" ischemia in canine hearts (223). Vinculin, like desmin and non-erythrocyte spectrin (152), is one of the components of the cytoskeletal attachment complex between the plasma nembrane and the Z-lines of the underlying myofibrils. In other experiments, using scanning electron microscopy, breakage of Z-band plasmalemmal attachment complexes could only be observed after periods of ischemia longer than 120 minutes (179). These findings suggest that subtle cytoskeletal alterations might be involved. Further investigation of these possible mechanisms requires immunolabeling at the electron microscopical level.

In our laboratory we have performed pilot studies to investigate the possible involvement of cytoskeleton disorders during myocardial ischemia. To this end we have been able to develop an immunolabeling protocol, applying cryo-ultramicrotomy to the labeling of desmin with the protein A-gold technique. This technique can be used to study the influence of ischemia on membrane-cytoskeleton interactions.

\subsection{Membrane protein disorders and cell damage.}

Originally attempts were made to investigate the involvement of ( $\mathrm{Na}, \mathrm{K})$ ATP'ase in ischemia and reperfusion induced membrane damage, since intramembranous particles. as observed with freeze-fracture electron microscopy, are supposed to be largely composed of (Na,K)ATP'ase (132), and these particles are found to aggregate under conditions of ischemia and reperfusion $(7,8,80,83,164)$.

Furthermore, ATP depletion, as observed during myocardial ischemia, can lead to the backward running of the (Na, K)ATP'ase (10). Under these conditions (Na,K)ATP'ase retains it $\mathrm{Na} / \mathrm{K}>1$ stoichiometry and so generates an inward current (II). which may 
lead to disturbances in the excitability of cardiac sarcolemma.

To this end polyclonal antisera were raised against (commercially obtained) (Na,K)ATP'ase from dog kidney, which appeared to specifically react with the alphasubunit of the enzyme. Antisera against (Na,K)ATP"ase (kindly provided by Prof. De Pont, Nijmegen), which were produced in goats immunized with purified enzyme or enzyme subunits from rabbit kidney, have also been applied. Both groups of antisera gave similar immunofluorescent labeling of sarcolemma and intercalated discs with occasionally faint, spotty labeling of mitochondria in ( $4 \mu \mathrm{m}$ cryo-section of) cardiac tissue (190).

However, investigation of the aggregation of $(\mathrm{Na}, \mathrm{K}) \mathrm{ATP}$ 'ase at the electron microscopic level was encountered by insuperable problems, since the preservation of the antigenicity of (Na,K)ATP'ase was incompatible with any of the thusfar tested preparative procedures for preservation of the ultrastructure. Alternatively ultrastructural labeling of (Na.K)ATP'ase may be obtained by affinity-gold labeling (146). Colloidalgold affinity probes prepared with ouabain, a selective inhibitor of $(\mathrm{Na}, \mathrm{K}$ )ATP'ase, or the novel steroid derivative prednisolone-3,20-bisguanyl hydrazone (PBGH), with a high affinity for cardiac (Na,K)ATP'ase isoenzymes (153), may be used as the directing ligands to investigate the ultrastructural localization of this enzyme complex in cell membranes.

Membrane protein disorders might be more important than generally believed thusfar. Recently the fundamental concepts concerning the regulation of cellular membrane structure and function by membrane lipids has been reviewed by Curtain el al. (53). On the other hand, the membrane content of membrane proteins might well be larger than generally accepted (265). This may be specially important because of the functional interactions between membrane proteins and lipids. The protein content of most cellular membranes might well be sufficiently high to occupy the membrane surface as extensive or even more than the surface area occupied by the lipids in the bilayer (265). Hence, membrane proteins might contribute to membrane structure and function in a more important way than has been accepted so far. 


\subsection{Closing remark.}

Further investigation of possible (biochemical and/or physico-chemical) mechanisms of ischemia and/or reperfusion induced membrane destabilization, as might be reflected by the formation of multilamelfar vesicles couid shed more light on the development of myocardial cell injury. To this end not only changes in lipid content and composition should be examined, but also the interaction between phospholipids. on the one hand, and the glycocalyx and the cytoskeleton on the other. It also needs to be established whether membrane alterations as studied thusfar are the cause or merely one of the many consequences of irreversible myocardial ischemic injury (242). 



\section{REFERENCES}

1. Alshire A, Piper HM. Cuthbertson KSR. Cobbold PH (1987) Cytosolic free calcium in single frat heart cells during anoxia and reoxygenation. Biochem $J$ 244: $381-385$.

2. Alberts B. Bray $\mathbb{D}$. Lewis $J$, Roff $M$, Roberts $K$. Watson $\mathbb{D}$ (1983) In: Molecular biology of the cell. Garland Publ Inc. New York. pp: 1-1146.

3. Angermulter S. Fahimi HD (1982) Imidazole-buffered osmium tetroxide: an excellent stain tor the viswalization of lipids in transmission electron microscopy. Histochem $.14: 823-835$.

4. Arroyo CM. Kramer $\mathrm{JH}$, Dickens $\mathrm{BF}$. Weglicki WB (1987) Identification of free radlicals in myocardial ischemia/reperfusion by spin trapping with nitrone DMPO. FEBS Letters 221: 101104.

5. Asani $\mathrm{K}$ (1986) Preservation of ultrastructure in phosphatidylcholine vesicles foy tannic acid and $\mathrm{OsO}_{4}$. Ultrasiluct Res $95: 38-46$.

6. Ashavaid TF, Colvin RA, Messineo FC. MacAlister $T$. Katz AM (1985) Effects of faty acids on $\mathrm{Na}$ /Ca exchange in cardiac sarcolemmal membranes. J Mol Cell Cardiol 17: 851-861.

7. Ashraf M, Halverson CA (1977) Structural changes in the freeze-fractured sarcolemma of ischemic myocardium. Am I Pathol 88: 583-594.

8. Ashraf $M$ (1978) Ultrastructural alterations in the mitochondrial membranes of ischemic nyocardium as revealed by freeze-fracture technique. J Mol Cell Cardiol 10: 535-543.

9. Askin FB, Kuhn $\mathrm{C}$ (1971) The cellular origin of pulmonary surfactant. Lab Invest 25: 260268 .

10. Bahinski A, Nakao M, Gadsby DC (1987) Potassium translocation by the Na/K pump is voltage insensitive. Proc Natl Acad Sci USA 85: 3412 3416.

11. Bahinski $A$, Gadsby DC (1988) Currentwoltage relationship of the backward-running $\mathbb{N a}$ K $\mathbb{K}$ punp in isolated cells from guinetapig ventricle. In: Biology of isolated adtuli cardia myocytes. Eds. Clark WA. Decker RS. Borg TK, Elsevier Science Publl Co, pp 430 433.

12. Baker NJDeC. Reid C (1982) Lamellar bodies in activated platelets as revealled by the use of tamic acid and a comparison with the concentric laminar organelles of type fl pneumocyles. J Anat 135: 539-548.

13. Becker LC. Antbrosio G (1987) Myocardial consequences of tepenfusion. Prog Cardiovasc Dis $30: 23-44$.

14. Beller GA. Conroy J. Smith T (1976) 1schemia-induced alterations in myocardial (Na, K)ATPase and cardiac glycoside binding, J Clin Invest 57: $341-350$.

15. Belton JC, Branton D, Thomas HV. Muellen PK (1971) Freeze-etch observations of rat lung. Anat Rec 170: 471-484.

16. Benthan JM. Higgins AJ. Woodward B (1987) The effects of ischemia. lysophosphatidytcholine 
and palmitoylcamine on rat hear phospholipase $A_{2}$ activity. Basic Res Cárdiol 82 (Suppt. 1): $127-136$.

17. Bergmeyer HU, Bernt $\mathbb{E}(1974)$ UV-assay for lactate dehydrogenase with pyruwate and NADH. In: Meihods of enzymatic analysis. Ed. Bergmeyer HU, Verlag Chemie GmbH, Weinhein., Vol 2, pp 57.579 .

18. Bersohn MM. Philipson KD. Fukushima JY (1982) Sodium-calcium exchange and sarcolemmal enzymes in ischemie rabbit hearts. Am J Physioll 242: C288-C295.

19. Blanchette-Mackie EJ, Scow RO (1981) Lipolysis and lamellar structures in white adipose tissue of young rats - Lipid movement in menbranes. J Ulirastruct Res 77: 295-318.

20. Blanchette-Mackie EJ, Scow RO (11983) Movenent of lipolytic products to mitochondria in brown adipose tissue of young rats: an electron microscope study. J Lipid Res 24: 229-244.

21. Blancliette-Mackie EJ, Amende LM (1987) Electron microscopic wisualization of fatry acids in tissues. J Ellectron Micr Tecthn 7: 205-221.

22. Borgers M, Piper HM (1986) Calcium-shifts in anoxic cardiac nyocytes. A cytochemical study. J Mol Cell Cardiol $18: 439-448$.

23. Borovjagin VL, Vergara IA, McIntosh TI (1982) Morphology of the intermediate stages in the lamellar to hexagonal lipid phase transition. J Membr Biol 69: 199-212.

24. Boyles I (1983) The use of primary amines to improve glutaraldehyde fixation. In: The science of biological specimen preparation. Ed. O'Hare AMF, SEM, Chicago, pp: 7-21.

25. Boyles J. Anderson L. Hutcherson $\mathbf{P}$ (1985) A new fixative for the preservation of actin filamenis. J Histochem Cyrochem 33: 1116-1128.

26. Braunwald E. Kloner RA (1982) The stunned nyocardium: Prolonged, post ischennic ventricular dysfunction. Circulation 66: $1146-1149$.

27. Bricknell OL. Opie LH (1978) Effects of substrates on the vissue metabolic changes in the isolated rat heart during underperfusion and on release of lactate deltydrogenase and arrhythmias during reperfusion. Circ Res $43: 102-115$.

28. Brightman MW. Reese TS (1969) Junctions between intimately apposed cell membranes in the vertebrate brain. I Cell Biol 40: 648-677.

29. Buja LM, Hagler HK, Parsons D, Chien $K$, Reynolds $R C$, Willerson JT (1985) Alterations of ultrastructure and elemental composition in cultured neonatal rat cardiac myocytes after metabolic inbibition with iodoacetic acid. Lab Invest $53 ; 397-412$.

30. Bullock GR. Christian RA, Peters RF. White AM (1971) Rapid mitochondrial enlargement in muscle as a response to triamcinolone acetomide and its relationship to the ribosomal defect. Biocthen Pharmacol 20: 943-953.

31. Burn P (1988) Amphitropic proteins: a new class of membrane proteins. Trends Biochem Sci 13: 79-83. 
32. Burton KP. Templeton GH. Hagler HK. Willerson JT. Buja LM (1980) Effect of glucose availability on functional membrane integrity. ultrastrueture and contractle performance following hypoxia and reoxygenation in isolated feline cardiac muscle. I Mol Cell Cardiol 12. $109-133$.

33. Burton KP, Hagler HK, Willerson JT, Buja LM (1981) Abnormal lanthanum accumulation due to ischemia in isolated myacardium: eftects of chloropromazine. Am J Physiol 241: H714-723.

34. Burton KP. Buja LM, Sen A, Willerson JT, Chien KR (1986) Accumulation of arachidonate in triacylglycerols and unesterified fatty acids during ischemia and reflow in the isolated rat heart. Am J Pathol 124: 238-245.

35. Caroni $\mathbb{P}$, Carafoli $E$ (1981) Regulation of $\mathrm{Ca}^{2+}$-pumping ATPase of heart sarcolenma by a phosphorylation-dephosphorylation process. J Biol Chem 256: $9371-9373$.

36. Carafoli $\mathrm{E}$ (1985) The homeostasis of calcium in heart cells. J Mol Cell Cardiol 17: 203. 212.

37. Carafoli E (1987) Intracellular calcium homeostasis. A Rev Biochem 56: 395-433.

38. Chandler WK, Schneider MF, Rakowski RF, Adrian RH (1975) Charge movements in skeletall muscle. Phil Trans R Soc London B270: 501-505.

39. Chapman D. Gonez-Fernandez JC, Goni FM (1979) Intriusic protein-lipid interactions. FEBS Letters $98: 211-223$.

40. Chatelain $P$, Brotelle $R$, Laruel $R$ (1988) Ischaemia reduces rat heart plasmalogens. I Mol Cell Cardiol 20 (Suppl. V): S66.

41. Cherry RJ, Muller U, Holenstein C. Heyn MP (1980) Lateral segregation of protein induced by cholesterol in bacteriorhodopsin-phospholipid vesicles. Biochim Biophys Acta 596: 145-151.

42. Chien KR, Pfau RG, Farber JL (1979) Ischemic myocardial cell injury. Prevention by chlorpromazine of an accelerated phospholipid degradation and associated membrane dysfunction. Am J Pathol 97: 505-529.

43. Chien KR, Reeves JP, Buja M, Bonte F. Parkey RW, Wilterson JT (1981) Phospholipid altera. thons in canine ischemic myocardium. Circ Res 48: 711-719.

44. Chien KR, Han A, Sen A, Buja LM. Willerson JT (1984) Accumulation of unesterifted arachidonic acid in ischemic canine myo-cardium. Circ Res 54: 313-322.

45. Chien KR, Sen A, Reynolds R, Chang A, Kim Y, Gunn MD, Buja M, Willerson JT (1985) Release of arachidonate from membrane phospholipids in cultured neonatal rat myocardial cells during adenosine triphosphate depletion: correlation with the progression of cell injury. $j$ Clin Invest 75: 1770-1780.

46. Collel AJ (1979) Preservation of alveolar type II pneumocyte lanellar bodies for electron microscopic studies. J Histochem Cytochem 27: 989-996.

47. Copeland BR, McConnell HM (1980) The rippled structure in bilayer membranes of phosphatidylcholine and binary mixtures of phosphatidylcholine and cholesterol. Biochim Biophys Acla 599: 95-109. 
48. Corr PD, Snyder DW. Cain ME. Crafford WA. Gross RW. Sobel BE (1981) Electrophysiological effects of amphiphiles on canine purkinje fibers: Imptications for dysthythmia secondary to ischemia. Circ Res $49: 345-363$.

49. Corr PB, Gross RW. Sobel BE (19:2) Arrhythogenic amphiphilic lipids and the myocardial cell membrane. I Moll Cell Cardial 14:619-626.

50. Corr PE. Gross RW, Sobel BE (1984) Amphipathic metabolites and nembrane dysfunction in ischenic myocardium. Circ Res 55: $135-154$.

51. Cowan JC. Vaugham Willams EM (1980) The effects of various fatty acids on action potential shortening during sequental periods of ischemia and reperfusion. I Mol Cell Cardiol 12: 347.369 .

52. Crevey BJ. Langer GA, Frank IS (1978) Role of Ca maintenance of rabbit myocardial cell membrane structural and functional integrity. J Mol Cell Cardiol 10: 1081-1100.

53. Curtain CC. Gordon LM, Aloia RC (1988) Lipid domains in biological membranes: conceptual devellopment and significance. In: Lipid domains and the relationship to nembrane function. Ed. Alan R, Liss Inc. pp: 1-15.

54. Das DK. Engelman RM, Rouson IA, Breyer RH. Otani H, Lemeshow S(1986) Role of membrane phospholipids in myocardial injury by ischemia and reperfusion. Am J Physiol 251: H71-H79.

55. De Bruijn WC (1973) Glycogen, its chemistry and morphollogical appearance in the electron microscope. I. A modified $\mathrm{OsO}_{4}$ fixative which selectively contrasts glycogen. I Ultrastruct Res 42: $29-36$.

56. De Bruijn WC. Den Breejen P (1975) Glycogen, its chemistry and morphological appearance in the electron microscope. II. the complex formed in the selective contrast staining of glycogen. Histochem J 7: 205-229.

57. De Brujin WC, Den Breejen P (1976) Glycoger, its chemistry and morphological appearance in the electron microscope. III. Identification of the tissue ligands imwolved in the glycogen contrast staining reaction with osminm(VI)-iron(II) complex. Histochem $₫ 8: 121-142$.

58. De Bruijn WC, Van Buitenen JMH (1980) X-ray microanalysis of aldellyde fixed glycogen contrast-stained by Os(VI).Fe(II) and Os(VI).Ru(IV) complexes. I Histochem Cylochem 28: 1242.1250 .

59. De Leiris J. Fewray $\mathrm{D}$ (1977) Ischenua-induced damage in the working rat heart preparation: The affect of perfusate substrate composition upon subendocardial ultrastructure of the ischemic left ventricular wall. $\mathrm{J}$ Mol Cell Cardiol 9: 365-373.

60. De Leiris J, Opie L.H (1978) Effect of substrates and coronary artery ligation on mechanical performance and on release of lactate dehydrogenase and creatine phosphokinase in isolated working rat hearts. Cardiovasc Res 12: 585-596.

61. De Leiris J. Hearse Dy (1984) Myocardial enzyme leakage as an indicator for cellular injury: Principles and applications. In: Methods in studying cardiac membranes. Ed. Dhalla NS, CRC Press. FLorida. Vol 1, pp: 253-278. 
62. Dermer GB (1970) The pulmonary surfactant content of the inclusion bodies found within type II alveolar cells. J Ultrastruct Res 33: 306-317.

63. Douglas WHJ, Redding RA, Stein M (1975) The lamellan structure of osmuiophilic inchusion bodies present in rat type II alveolar pneumocytes. Tissue \& Cell 7: 137-142.

64. Dubochet J. Lepault J, Freeman R, Berriman JA, Homo J-C (1982) Electron microscopy of frozen water and aqueous solutions. J Microsc (Oxford) 128: 219-237.

65. Dubochet J. Adrian M, Chang J-J, Lepaut J, MacDowall AW (1987) Cryo-electron nnicroscopy of vitrified specimens. In: Cryotechniques in biological electron microscopy. Eds "Steinbrecht RA. Zierold K. Springer Verlag. Berlin. pp: 114-131.

66. Dunn MJ (1974) Red blood cell calcium and magnesium: effects upon sodium and potassium transport and transport cellular morphology. Biochim Biophys Acta 352: 97-116.

67. Duzgunes N, Hong K. Baldwin PA, Beutz J, Nur S, Papalladjoponlos D (1987) Fusion of pliosphom lipid vesicles induced by divalent cations and protons - Modulation by phase transitions. free fatty acids. monovalent cations, and polyamines. In: Cell fusion. Ed. Sowers AE, Plenum Press. New York. pp: $241-267$.

68. Elbers PF, Ververgaert PHJT, Demel R (1965) Tricomplex fixation of phospholipids, J Cell Biol 24: 23-30.

69. Endo $M$ (1985) Calcium release from sarcoplasmic reticulum. In: Current topics in membranes and transport. Ed. Bonner F. Acadi Press. New York. Vol 25. pp: 181-230.

70. Estep TN. Mountcastle DB. Biltonen RL. Thompson TE (1978) Studies on the anomalous behavion of aqueous dispersions of dipalmitoytphosphatidyllkoline-cholesterol mixtures. Biochemistry 17: 1984-1989.

71. Fabiato A (1985) Calcium-induced release of calcium from the sarcoplasmic reticulum. I Gen Physiol 85: 189-320.

72. Farber JL, Chien KR, Mittnacht $S$ (1981) The pathogenesis of irreversible cell injury in ischemia. Am J Pathol 102: 271-281.

73. Feurray D. De Leiris $J$ (1975) Ultrastructural modification induced by reoxygenation in the anoxic isolated rat heart perfused without exogenous substrate. $J$ Mol Cell Cardiol 7: 307. 314.

74. Finch SAE. Piper HM "Spieckermann $\mathbb{P G}$, Stier A (1985) Anoxia influence the lateral diffusion of al lipid probe in the plasma menbrane of isolated curdiac unyocyles. Basic Res Cardiol 80 (Suppl. 1): 149.152.

75. Fisher AB, Chandler A (1984) Lung surfactant - Phospholipids and apoproteins. Exp Lung Res 6: $171 \cdot 174$.

76. Flameng W, Daenen W, Borgers $M$. Thone F, Xhonneux R, Van der Water A, Van Belle H (1981) Cardioprotective effects of lidoflazine during I-bour normothermic global ischenia. Circulation 64: 796-807. 
77. Fox KAA, Bergmann SR. Sabel BE (1985) Pathophysiology of myocardial reperfusion. Ann Rev Med 36: 125-144.

78. Frank 15 , Langer GA (1974) The myocardial interstitum: its structure and its role in ionic exchange. I Cell Buol 60: $586-601$.

79. Frank IS. Langer GA. Nudd LM. Seraydarian K (1977) The myocardial cell surface: its histochemistry and the effect of sialic acid and calcium removal on the structure and ionic exchange. Cire Res 41: 702-714.

80. Frank JS, Beydler S. Kreman M. Rau EE (1980) Structure of the freeze-fractured sarcolemma th the normal and anoxic rabbit myocardium. Circ Res $47: \| 31-143$.

81. Frank IS, Rich TL, Beydler S. Kreman $M$ (1982) Calcium depletion in rabbit myocardium. Ultrastructure of the sarcolemma and correlation with the calcium paradox. Circ Res. 51: 117.130.

82. Frank IS (1983) Ca depletion of the sarcolemma - ultrastructural chamges. Eur Heart $J$ 4(Suppl.H): 23-27,

83. Frank JS, Beydler S, Wheeler N. Shine KI (1988) Myocardial sarcolemma in ischemia: a quantitative freeze-fracture study. Am J Physiol 255: H467-H475.

84. Franzini-Armstrong $\mathrm{C}$ (1986) The sarcoplasmatic reticulum and the transverse tubules. In; Myollogy. Eds. Engel AG. Banker BQ. MoGraw-Hill, New York. pp: 125-154.

85. Frederik PM, Stuart MCA, Bomans PHH "Busing WM (1989a) Phospholipid, nature's own slide and coverslip for cryo-electron microscopy. I Microsc (Oxford) $153: 81-92$.

86. Frederik PM. Stuart MCA, Schrijwers AHGJ, Bomans PHH (1989b) Thin film formation and the imaging of phospholipid by cryo-electron nicroscopy. In: The science of biological specimen preparation. Ed. O'Hare AMF, SEM (in press).

87. Gamache DA, Hess HL. Franson RC (1987) Phospliolipid alterations in canine cardiac sarcoplasnatic reticulum induced by an acid-active phospholipase C. Basic Res Cardiol 82 (Suppl. $1): 113-119$

88. Ganote $C E$, Seabra-Gomes $\mathbb{R}$. Nayler WG. Jennings $R B$ (1975) Irreversible myocardial injury in anoxic perfused rat hearts. Am J Pathol 80:919-938.

89. Ganote GE (1983) Contraction band necrosis and irreversible myocardial injury. I Mol Cell Cardiol 15: 67-73.

90. Ganote CE, Van der Heide RS (1987) Cytoskeletal lesions in anoxic myocardial injury. Am $J$ Pathol 129:327-344.

91. Ganote CE, Van der Heide RS (1988) Irreversible injury of isolated adult rat myocytes. Am J Pathol 132: 212-222.

92. Gigg R, Payne S (1969) The reaction of glutaraldehyde with tissue lipids. Chem Phys Lipids 3: $292-295$. 
93. Gil J (1972) Effect of tricomlex fixation on lung lissue, J Ultrastruct Res 40: 122-131.

94. Gil J. Reiss OK (1973) Isolation and characterization of lamellar bodies and hulvular myelion from rat lung homogenates. $J$ Cell Biol 58: 152-171.

95. Glauert AM (1974) Fixation, dehydration and embedding of biological specimens (Chap. 2 , Fixatives). in: Practicle methods in electron nicroscopy. Ed. Glatert AM. North-Holland Publ Comp. Amsterdam. pp: 5-72.

96. Goldstein MA, Thyrum PT, Murphy DL, Martin JH, Schwartz A (1977) Ultrastruchural and contractile characteristics of isolated papillary muscle exposed to acute liypoxia. I Mol Cell Cardiol 9: 285-295.

97. Granger DN, Hîlwarth ME, Parks DA (1986) Ischemia-reperfusion injury: role of oxygenderived free radicals. Acta Physiol Scand 548: 47-63.

98. Hallman M. Miyai K. Wagner RM (1976) Isolated lamellar bodies from ral lung - Correlated ultrastructural and biochemical studies. Lab Invest 35: 79-86.

99. Hardy PM. Hughes GJ Rydon HN (1976) Formation of quaternary pyridinium compounds by the action of glutaraldehyde on proteins. J Chem Soc Chem Comm 157.158.

100. Harper IS, Lochner A (1989) Sarcolemmal integrity during ischemia and reperfusion of isolated rat heart. Basic Res Cardiol 84: 208-226.

101. Hattori M, Ogawa K. Satake T, Sugiyana S, Ozawa T (1985) Depletion of membrane phospholipid and mitochondrial dysfunction associated with coronary teperfusion. Basic Res Cardiol 80: $241-250$.

102. Hauser H, Phitlips MC (1979) Interaction of the polar groups of phospholipid bilayer membranes. In: Progress in surface and membrane science. Vol, 13. Eds. Cadenhead DA, Danielli JF. Academic press, New York. pp: 297-413.

103. Hayar MA (1981) Aldehydes: Glutaraldehyde-tannic acid. In: Fixation for electron microscopy. Acad Press, London. pp: 124-129.

104. Hearse DJ. Humphrey SM. Nayler WG. Slade A. Border D (1975) Vltrastructural damage associated with re-oxygenation of the anoxic myocardium. J Mol Cell Cardiol $7: 3154324$.

105. Hearse DJ, Humphrey SM. Bullock GR (1978) The oxygen paradox and the calcium paradox: Two facets of the same problem? J Mol Cell Cardiol 10:641-668.

106. Hearse DJ (1979) In: Enzymes in cardiology: Diagnosis and research. Eds. Hearse DI and De Leiris J. John Wiley \& Sons, Chichester. pp: 1-19.

107. Houslay MD. Stanley KK (1982) In: Dynamics of biological membranes, John Wiley \& Sons. New York. pp: 1-130.

108. Hui S-W. Parsons DF (1975) Direct observations of domains in wet lipid bilayers. Science 190: $383-384$. 
109. Hulsmann WC, Dubeliar M-L (1987) Early damage of vascular endothelium during cardiac ischemia. Cardiowase Res 21:674-677.

110. Humphrey SM. Thomson RW. Gavin JB (1984) The influence of the no-reflow phenomenon on reperfusion and reoxygenation tamage and enzyme release from anoxic and ischemic isolated rat hearts. J Mol Cell Cardiol 16: 915-930.

111. Hunter DR, Haworth RA. Southard JH (1976) Relationship between configuration, function, and permeability in calcitw-treated mitochondria. J Biol Chem 251: 5069-5077.

112. 1keda H (1985) An ultrastructural analysis of the inclusion body in the type II pneumocyte processed by rapid freezing followed by freeze-substitution - An autoradiographic study. J Eleciron Microse 34: 398-410.

113. Jacobson BS (1983) Interaction of the plasmamembrane with the cytoskeleton: an overview. Tissue \& Cell 15: $829-852$.

114. Jennings RB, Ganote ChE (11974) Structural changes in myocardium during acute ischemia. Circ Res 34-35 (Stuppl. III): 156-168.

115. Jennings RB, Ganote ChE (1976) Mitochondrial structure and function in acute myocardial ischemic injury. Circ Res 38 (Suppl, 1): 80-91.

116. Jennings AB, Hawkins HK, Lowe JE, Hill ML, Kloman S, Reiner KA (1978) Relation berween high energy phosphate and lethal injury in myocardial ischemia in the dog. Am J Pathol 92 : $187-214$.

117. Jennings RB, Reimer KA (1981) Lethal myocardial ischemic injury. Am J Pathol 102: $241-255$.

118. Jennings RB, Reimer KA (1983) Factors involved in salvaging ischemic myocardium" effect of reperfusion of arterial blood. Circulation 68 (Suppl. 1)." 125-136.

19. Jennings RB, Steenbergen C. Kinney RB. Hill ML, Reimer KA (1983) Comparison of the effect of ischaemia and anoxia on the sarcolemma of the dog heart. Eur Heart J 4 (Suppl.H): 123137.

120. Jennings RB. Schaper J, Hill M. Steenbergen C, Reimer KA (1985) Effects of reperfusion late in the phase of reversible ischemic imjury: changes in cell volume, electrolytes, metabolites. and ultrastructure. Circ Res 56: 262-278.

121. Jennings RB, Reimer KA. Steenbergen $C$ (1986) Myocardial ischemia revisited. The osmolar load, nembrane damage, and reperfusion. J Mol Cell Cardiol 18: 769-780.

122. Johnson TJA (1983) Glutaraldehyde fixation chenistry. A sclweme for rapid crossulinking and evidence for rapid oxygen consumption. In; The science of biological specimen preparation. Ed O'Hare AMF. SEM. Chicago. pq: 51-62.

123. Jost $P$, Brooks UJ, Griffith OH (1973) Fluidity of phospholipid bilayers and membranes after exposure to osmium tetroxide and glutaraldehyde. J Mol Bioll 76:313-318.

124. Kalina M. Pense DC (1977a) The preserwation of ultrastructure in saturated phosphatidyl cholines by tannic acid in model systems and type II pneunocytes. J Cell Biol 74: 726-741. 
125. Kalina $M$. Pease DC (1977b) The probable role of phosphatidyi cholines in the tannic acid enhancenemt of cytomembrane electron contrast. J Cell Biol 74: 742-746.

126. Karnovsky MJ (1971) Use of ferrocyanide reduced osmiun tetroxide in electron microscopy. Proc. 14th Ann Meet An Soc Cell Biol. p. 146 a.

127. Katz AM, Messineo FC (1981) Lipidmembrane interactions and the pathogenesis of ischemic damage in the myocardium. Circ Res 48: 1-16.

128. Kawaguchi $H_{n}$ Yasuda $H$ (1988) Prostacyclin biosynthesis and phospholipase activity in llypoxic rat myocardium. Circ Res 62: 1175-1181.

129. Kloner RA, Ganote ChE, Whallen DA. Jennings BS and RB (1974) Effect of a transient period of ischemia on myocardial cells. II.Fine siructure during the first few minutes of reflow. Am J Pathol 74: 399-422.

130. Kloner RA, Braumwald E (1980) Observations on experimental myocardial ischemia. Cardiovasc $\operatorname{Res} 14: 371 \times 395$.

131. Kloner RA, Ellis SG. Lange R. Braunwald E (1983) Studies of experimental coronary arlery reperfusion. Effects of infarct size, myocardial funcrion, biocllemistry, ultrastructure and microvascular damage. Circulation 68 : 18-115.

132. Kordylewski L, Karrison $T$, Page E (1985) Measurements on the internal structure of freezefractured cardiac plasma membrane. Am II Physiol 248: H297-304.

133. Krause S, Hess ML (1984) Characterization of cardiac sarcoplasmic reticulum dysfunction during shortterm, normothermic, global ischemia. Circ Res 55: 176-184.

134. Krueger ChM, Neufeld EJ. Saffitz JE (1985) Preservation of arachidonoyl phospholipids during tissue processing for electron microscopic autoradiography. I Histochem Cytochem 33: $799-802$.

135. Kuhn C (1972) A comparison of freeze-substitution with other methods for preservation of the pulmonary alveolar lining layer. Am J Anat 133: 495-508.

136. Langendorff $O$ (1895) Untersuchen am uberlebenden Saugetierherzen. Pflugers Arch Physiol 61: $291-332$.

137. Langer GA, Serena SD, Nudd LM (1974) Cation exchange in hear cell cullure: correlation with effects on contractile force. J Mol Cell Cardiol 6: 149-161.

138. Langer GA (1978) The structure and function of the myocardial cell surface. An IJ Physiol 235: $\mathrm{H} 4461-\mathrm{H} 468$.

139. Lepault J. Pattus F. Martin N (1985) Cryo-electron microscopy of arnificial biological membranes. Biochim Büophys Acta 820:315-318.

140. Lochner A, Sanan D. Victor T, Bester R. Kotze JCN, Van der Merwe N, Shabon 1 (1985) In: Membrane and muscle. Eds. Berman MC. Opie LH. IRL Press, Oxford. pp: 309-325. 
141. Magoon MW, Wrigh JR. Bariussio A. Willatrs MC Goeke J. Benson BI. Hanilton RC. Clermentw JA (1983) Subfractionation of lung surfactant, irnplicatrons for metabolism and surfice activity. Brochm Blophys Acta $750: 18-31$.

142. Manonos AN (1984) Mechanisms of $\mathrm{Ca}^{2}$ release from sarcoplasmic renticulun of skelletal muscle. PHysiol the 64:1240-1320.

143. MCNun NS, Fawcen DW (1974) Myocardial ultrastructure, In: The mamualian myocardium. Eds. Langer GA and Brady AJ. John Wiley \& Sons. New York. pp: $1-49$.

144. Miyazak $X$. Nagai S. Ogaud K. Satake T. Sugiyana S. Ozawa T (1984) The role of phosphom lipase in mitochondral dysfunction after cotonary reperfusion in the canine nyocardium. Jap Ciric $48: 498-507$.

145. Mizulura $\mathrm{V}$. Futaesaku $\mathrm{Y}(1971)$ On the new approaclu of tannic acid and digitonin to the biological fixatiwes. Proc Electron Miciose Soc Am 29: 494-495.

146. Mrsny $\mathrm{kJ}$, Burell GB, Volwerk JJ, Widdicombe JH. Guffith OH (1987) A novel spproach to the ultastructural localization of cell surface receptols: affiritygoldlabeling of (Na, K)ATP. aste. Eutu I Cell Biol 45: 200-208.

147. Muir AR (1968) A calcium-induced contracture of cardiac muscle cells. I Anat 102:148-149.

148. Nyyler WG (1981) The nole of calcium in the ischenic nyocarditum. Am J Pathol 102: 262-270.

149. Neely JR, Grotyohann LW (1984) Role of glycolytic products in damage to ischemic myocarditum. Dissociation of adenosine triphosphate levels and recovery of function of reperfused iscliemic hearts. Circ Res 55:816-824.

150. Neelly JR, McDonough KH (1984) Factors that influence myocardial levels of long ochain acyl CoA and acyl carnitine. In: Myocardial ischemia and lipid metabolism. Eds. Ferrari R, Katz AM, Visioli O. Plenum Press, New York. pp: 159-169.

151. Neiss WF (1984) Electron staining of the cell surface coat by osmium-low ferrocyanide. Mistochum $80: 231-242$.

152. Nelson WJ, Lazarides E (1984) Assembly and establishent of membrane cytoskeleton domains during differentiation: Spectrin as a model-system (Ch. 6). In: Cell membranes. Methods and reviexts, Vol. 2. Eds. Elson E. Frazien W, Glaser L. Plenum Press. New York. pp: 219-246.

151. $\mathrm{Ng} \mathrm{Y}-\mathrm{C}$. Leung W-Y. A.kera T (1988) Cardiac Na,K-ATPase isoenzymes: sensilivity to prednisolone bisgunnylhydrazone. Eur I Pharamcol 155: 99-99.

154. Nickerson PA (1983) Lipid droplets in the adrenal contex of the rat. Preservation after tannic acid-paraformaldehyde-glutaratdelyde fixation and extraction during staining. Tissue \& Cell 15: $975-982$.

155. Oldfield F. Chapman D (1972) Dynamics of lipids in membranes: Heterogeneity and the role of cholesterol. FEBS Lett $23: 285-297$.

156. Op den Kamp JAF (1979) Lipid asymmetry in membranes. A Rev Biochem 48: 47-71. 
157. Ortega A. Mas-Oliva J (1984) Cholesterol effect on enzyme actinity of the sarcolenmal (Ca,Mg)ATPase from cardiac muscle. Biochim Biophys Acta 773: 231:236.

158. Otani H, Engelman RM, Rousou JA, Breyer RH, Das DK (1986) Enhranced prostaglandin synthesis due to phospholipid breakdown in ischemic-reperfused myocardium. I Mol Cell Cardiol 18 : 953-961.

159. Paulson DI. Traxler J. Schmict M. Noonan J. Shug AL (1986) Protection of the ischemic myocardium by L-propionylcarnitine: effects on the recovery of cardiac output after ischaemia and reperfusion, carnitine transport. and fatty acid oxidation. Cardiovasc Res 20: $536-541$,

160. Pfeiffer DR. Schmid PC Beatrice MC. Schmid HHO (1979) Intramitochondrial phospholipase activity and the effects of Calcium plus $\mathrm{N}$-ethylmaleimide on mitochondrial function $\mathrm{J}$ Cell Biol 254: 11485-11494.

161. Piper HM, Schwartz P, Spahr R, Hutter JF, Spieckermann PG (1984) Early enzyme release from nnyocardial cells is no due to irreversible cell damage. J Mol Cell Cardiol 16: $385-388$.

162. Piper HM, Schwartz P. Spahr R, Hutter JF. Spieckermann PG (1985) Anoxic injury of adult cardiac myocytes. Bas Res Cardiol 80 (Suppl. 1): 37-41.

163. Piper HM (1988) Metabolic processes leading to myocardial cell death. In: Methods and achievements in experimental pathology. Ed Jasmin G, Karger Publ, Basel. pp: 144-180.

164. Post JA, Leunissen-Bijvelt J. Ruigrok TJC. Verkleij AI (1985) Ultrastluctural changes of sarcolemma and mitochondria in the isolated rabbit heart during ischemia and reperfusion. Biochin Biophys Acta 845: 119-123.

165. Post JA, Lamers JMJ, Verdouw PD, Ten Cate FJ, Van der Giesen WI, Verklleij AJ (1987) Sarcolemmal destabilization and destruction after ischaemia and reperfusion and its relation with long-term recovery of regional left ventricular function in pigs. Eur Heart $J$ 8: $423-430$.

166. Post JA, Langen GA. Op den Kamp JAF. Verkleij AJ (1988) Phospholipid asymmetry in cardiac sarcolemna. Analysis of intact cells and "gas-dissected" menbrane. Biochim Biophys Acta 943: 256-266.

167. Primen FW, Van der Yusse G. Aris T, Roemen THM. Coumans WA, Renteman RS (1984) Accumulation of nonesterified fatty acids in ischemic canine myocardium. Ans J Physiol 247: H264H272.

168. Rahamethulla PN, Ashraf $\mathrm{M}$ (1984) Heterogeneity of cell response to carly anoxia and reoxygenation in rat heart. Exp Pathol 25: 139-146.

169. Regitz V, Paulson DJ, Hodach RI. Little SE, Schaper W, Shug AL (1984) Mitochondrial damage during myocardial ischemia. Basic Res Cardiol 79: 207-217.

170. Reimer KA. Jennings RB. Hill ML (1981) Total ischemia in dog heart in vitro. 2. High energy phosphate depletion and associated defects in energy metabolism, cell volume regulation. and sarcolemmal integrity. Circ Res 49:901.911. 
174. Reimer KA, Jennings HB, Tatum AH (1983) Pathobiology of acute myocardial ischemia: Metabolic, functional and ultrastuctural studies. Am $J$ Cardiol 52: 72A-81A.

172. Richards FM, Knowles JR (1968) Glutaraldehyde as a protein cross-linking agent. J Mol Biol $37: 231-233$.

173. Rienersitu JC (1963) Osmum tetroxide fixation of lipids, nature of the reaction products, J Histochem Cytochem $11: 436-442$.

174. Roemen THM, Van der Vusse GJ (1985) Appication of silicagel column chronatography in the assesstinent of non-esterified fatty acids and phosphoglycerides in myocardial tissue. I Chromat 344: $304-308$.

175. Rogers $J$, Lee AG, Wilton DC (1979) The organization of cholesterol and ergosteroll in lipid bilayers based on studies using non-perturbing fuorescent sterol probes. Biochim Biophys Acta 552: 23-37.

176. Romero PJ (1974) The role of membrane bound magnesium in the permeability of ghosts to $\mathrm{K}^{+}$. Biochim Biophys Acta 339: 116-125.

177. Sabatini DD, Bensch K, Barrnett RJ (1963) Cytochemistry and cellulat ultrastructure and enzymatic activity by aldelyde firation. I Cell Biol 17: $19-58$.

178. Saffiz JE, Gross RW, Willtamson JR, Sobel BE (1981) Autoradiography of phosphatidyl choline. J Histochem Cytochen 29:371-378.

179. Sage MD, Jennings RB (1988) Cytoskeletal injury and subsarcolenmal bleb formation in dog heart during in vitro total ischemia. Am J Pathol 133: 327-337.

180. Sakai K. Gebhard MM. Spleckermann PG, Bretschneider HJ (1975) Enzyme release resulting from total ischemia and reperfusion in the isolated, perfused guinea pig heart. $\mathrm{J}$ Mol Cell Cardioll 7:827-840.

181. Sakakibara K, Momoi T, Uchida T, Nagai $X^{\prime}$ (1981) Evidence for association of glycosphingolipid will a colchicine-sensitive microtubule-like cytoskeletal structure of cultured cells. Nature 293: 76-77.

182. Sanderminn H (1978) Regulation of membrane enzymes by lipids. Biochim Biophys Acta 515: $209-237$.

183. Schaper J, Hehrlein F. Schlepper M, Thiedemann K.U (1977) Ultrastructural alterations during ischemia and reperfusion in human hearts during cardiac surgery. $J$ Mol Cell Cardiol 9: $175=189$.

184. Schaper J, Mulch J, Winkled $\mathbb{B}$, Sclaper W (1979) Ultrastructural, functional, and biochenical curturia for estimation of reversibility of ischemic injury: A study on the effects of global ischemia on the isolated dog heart. JMol Cell Cardiol 11:521-541.

185. Schaper J. Scheld HH, Schmidt U, Hehrlein F (1986) Ultrastructural study comparing the efficacy of tive different methods of intraoperative myocardial protection in human heart. J Thorac Cardiovasc Surg 92: 47-55. 
186. Schmalzing G. Kutschera $P$ (1982) Modulation of ATPase actiwities of human erythrocyte membranes by free fatty acids or phospholipase $\mathrm{A}_{2}$. J Membrane Biol 69: 65-76..

187. Schock C, Pattle RE, Creasey JM (1973) Methods for electron microscopy of the lamellated osmiophillic bodies of the lung. $y$ Microsc (Oxford) $97: 321-330$.

188. Schrijvers AHGJ, Snoeckx LHEH. Stuart MCA. Frederik PM, Reneman RS (1988a) Examination of glutaraldehyde-lysine fixed ischemic nyocardium. J Mol Cell Cardiol 20 (Suppl. V) S3l.

189. Schrijwers AHGJ. Frederik PM, Heijnen VWTh, Reneman RS (1988b) Increased number of multilamellar extrusions after prolonged ischenia and reperfusion in rabbit myocardium. $J$ Mol Cell Cardiol 20 (Suppl. V): $\$ 31$.

190. Schrijvers AHGJ, Heijnen VVTh, Frederik PM, Reneman RS (1988c), Immunocytochenical localization of (Na.K)ATP"ase in cardiac tissue. Ultramicroscopy $24: 447$.

191. Schrijwers AHGJ, Frederik PM, Stuart MCA, Renenan RS (1988d) The reaction of tannic acid with phospholipids - A fixation or modification of the ultrastructure. I Mol Cell Cardiol 20 (Suppl. V): S76.

192. Schrijwers AHGJ. Snoeckx LHEH, Stuart MCA, Frederik PM, Reneman RS (1988e) Glutaraldehydelysine fixation for studying cardiac membranes, Ultramicroscopy $24: 447$.

193. Schrijvers AHGJ, Frederik PM, De Groot MJM, Heijnen VVTh, Van der Vusse GJ, Reneman RS (1989a) Induction of multilamellar vesicles in the isolated rabbit heart after ischenia and reperfusion. Ultramicroscopy 27: 216-217.

194. Schrijvers AHGJ, Frederik PM. Stuart MCA, Van der Vusse GJ, Reneman RS (1989b) Dual effect of tannic acid on the preservation and ultrastructure of phosphatidyl choline vesicles. Mol Cell Biochem 88: 91-96.

195. Schrijvers AHGJ, Frederik PM. Stuart MCA, Burger KNI, Heijnen VVTh, Van der Vusse GJ, Reneman RS (1989c) The formation of multilamellar vesicles by the addition of tannic acid to phosphatidylcholine containing snall unilamellar wesicles. I Histochem Cytochem (in press).

196. Schwertz DW, Halverson J, Isaacson T, Feinberg H, Palner JW (1987) Alterationis in phospholipid metabolism in the globally ischemic rat heart: emphasis on phosphoinositide specific phospholipase C activity. J Mol Cell Cardiol 19:685m697.

197. Scott RE, Perkins RG. Zschanke MA. Hoerl BJ, Maercklein PB (1979) Plasma menbrane vesiculation in $3 \mathrm{~T} 3$ and SV3T3 cells. 1. Morphological and biochemical characterization. I Cell Sci 35: $229-243$.

198. Sen A. Buja LM, Willerson JT. Chien KR (1987) Membrane phos-pholipid metabolism during myocardial ischaemia: past, present and future. Basic Res Cardiol 82 (Stppl.1): 121-125.

199. Shaikh NA, Downar E (1981) Time course of changes in porcine myocardial phospholipid levels during ischemia. Circ Res 49: 316-325.

200. Shen AC, Jennings $\mathbb{R B}$ (1972) Kinetics of calciun accumulation in acute myocardial ischemic injury. Am J Pathol 67: 441-452. 
201. Shine KL, Douglas AM. Ricchiut NV (1978) Calcium, strontium and baritum movements during ischemia and reperfusion in rabbit ventricle. Circ Res 43: 712-720.

202. Shug AL. Shrago E. Bitar N, Folts JD, Koke IR (1975) Acyl-CoA inhibition of adenine nucleotide translocation in ischemic myocardium. Am J Physio 228: 689-692.

203. Sinug AL. Thomessen JH, Folts JD, Bittar N. Klei MI, Koke JR, Huth PJ (1978) Changes in tissue levers of carnitine and other metabolites during myocardial ischemia and anoxia. Arch Biochem Biophys 187: 25-33.

204. Silva MT, Mota MMS. Melo JVC, Guerra FC (1971) Uranyl salts as fixatives for electron microscopy. Study of the membrane ultrastructure and phospholipid loss in bacilli. Biachin Biophys Acia 233: 513-520.

205. Simionescu $N$, Simionescu $M$ (1976) Galloylglucoses of low molecular weight as mordan in electron microscopy. I. Procedure and evidence for mordanting effect. J Cell Biol 70: $608-621$.

206. Singer SJ and Nicholson GL (1972) The fluid mosaic model of the structure of cell menbranes. Science 175: 720-731.

207. Smith AD, Subbs CD (1987) Modulation of membrane protein function by bilayer lipids. Bas Res Cardiol 82 (Suppl. I): 93-97.

208. Smith DS, Smith V, Ryan JW (1972) Freeze-fractured lamellar body membranes of the rat lung great alveolar cells. Tissue Cell 4:457-468.

209. Smutzer G, Yeagle PL (1985) A fluorescence anisotropy study on the phase behavior of dimyristoylphosphatidy/choline/cholesterol mixtures. Biochim Biophys Acta 814: 274-280.

210. Snoeckx LHEH, Van der Vusse GI. Coumans WA, Willensen PHM, Van der Nagel T, Reneman RS (1986) Myocardial function in normall and spontaneously hypertensive rats during reperfusion following a period of global ischemia. Cardiovase Res 20: 67-75.

211. Snoeck LHEY (1987) Ischemia tolerance of the hypertrophied rat neart. Ph.D. thesis, University of Limburg, Maastricht, The Netherlands.

212. Sobel BE, Corr PB, Robinson AK, Goldstein RA, Witkowski FX, Kilein MS (1978) Accunulation of Iysophosphoglycerides with arthythmogenic properties in ischemic myocardium. J Clin Invest 62: $546-553$.

213. Somlyo AP (1985m) Excitation-contraction coupling and the ultrastructure of smooth muscle. Circ Res 57: 497-507.

214. Somlyo AP (1985b) Excitation-contraction coupling. The messenger across the gap. Nature, Lonclon 316: 298-299.

215. Somlyo AP. Somlyo AV, Bond M. Broderick R, Goldman YE, Shuman H, Walker JW. Trentham DR (1987) Calcium and magnesium movements in cells and the role of inositol triphosphate in muscle. In: Cell calcium and the control of membrane transport. Eds Eaton DC, Mandel LJ. Rockefeller University Press. New York. Yol 24, pp: 77-92. 
216. Somlyo AV (1979) Bridging structures spaning the junctional gap at the triad of skeletal muscle. I Cell Bial 80: 743-750.

217. Sordaht LA. Stewart ML. (1980) Mechanism(s) of altered mitochondrial Calcium transport in acutely ischemic canine hearts. Circ Res 47: 814m820.

218. Sperelakis $\mathrm{N}$ (1986) Regulation of calcium-slow channels in myocardial cells by cyclic nucleotides and phosphorylation. In: Protein phosphorylation in heart muscle. Ed. Soltaro RJ. CRC Press . Florida. pp: 55-83.

219. Spieckermann PG. Nordbeck H. Preusse CJ (1979) In: Enzymes in Cardiology: Diagnosis and research. Eds. Hearse DJ and De Leiris J, John Wiley \& Sons, Chichester. pp: 81-95.

220. Stam H. Hulsmann WC (1985) Regulation of lipases involved in the supply of substrate farty acids for the heart. Eur Heart J 6:158:167.

221. Steenbergen C. Jennings RB (1984) Relationship between lysophospholipid accunulation and phasma membrane injury during total in vitro ischemia in dog heart. I Mol Cell Cardiol 16 : $605-623$

222. Steenbergen $C$, Hiill $M L$, Jennings $R B$ (1985) Volume regulation and plasma membrane injury in vitro. Effects on osmotic cell swelling on plasna membrane integrity. Circ Res 57: 864-875

223. Steenbergen C, Hill MI, Jennings RB (1987) Cytoskeletal damage durting myocardial ischemia: Changes in vinculin immunofluorescence staning during total in vitro ischemia in canine heart. Circ Res 60: 478-486.

224. Stein $O$, Stein $Y$ (1971) Light and electron microscopic autoradiography of lipids: techuiques and biological applications. Adv Lipid Res 9: 1-72.

225. Stoeckentus W, Mahr SC (1965) Studies on the reaction of osminm tetroxide with lipids and related compounds. Lab Invest 14: $458-469$.

226. Stratton CJ (1975) Muttilamellar body formation in mammalian lung. An ultrastructural study utillizing three lipid-retention procedures. Lung multilamellar body formation $J$ Uthastr Res 52: 309.320 .

227. Sugrue SP. Hay EA (1981) Response of basal epithelial cell surface and cytoskelelon wo solubilized extracellular matrix molecules. I Cell Biol 91 : $45-54$.

228. Takahashi $\mathrm{K}$, Kako KJ (1983) The effect of a calcium channel antagonist, nisoldipine, on the ischemia-induced change of cardiac sarcolemmal membrane. Bas. Res Cardiol 78: 326-337.

229. Tillney LG, Brain J, Bush DJ. Fujiwara K. Mooseker MS, Murphy DH, Snyder DH (1973) Microtubules - evidence for 13 protofilaments. J Cell Biol 59: 267.275.

230. Tranum -Jensen J. Janse M.I. Fiolet JWT, Krieger WJG. Naumann d'Alnoncourt C. Durrer D (1981) Tissue osmolarity. cell swelling, and reperfusion in acule regional myocardiat ischemia in the isolated porcine hear". Circ Res 49 : $364-38 \%$.

231. Traunwein W, Cawatie A, Flockerzi V. Hofmann F. Pelzer D (1987) Modulation of calcium channel function by phosphorylation in guinea pig ventricular cells and phosplinolipid bi- 
layer membranes. Cire Res 61 (Suppl. 1): 17-23.

232. Triggle Dr (1972) Effects of calcium on excilable menbranes and neurotransmitter action. In: Progress in surface and membrane science Vol.5. Eds. Danielli JF, Rosenberg M, Cadenhead DA, Academic press, New York. pp: 267-331.

233. Van Bilsen $M$ (1988) The significance of myocardial non-esterified fatty acid accumulation during ischemits and reperfusion. Ph.D. thesis. University of Limburg, Maastricht. The Netherlands.

234. Van Bilsen M. Van der Vusse GJ, Willemsen PHM, Coumans WA, Roemen THM, Reneman RS (1989) Lipid alterations in isolated, working rat likearts during ischemia and reperfusion: its relation to myocardial damage. Circ Res $64: 304 \% 314$.

235. Wan den Boscln H (1974) Phasphoglyceride metabolism. Annu Rev Biochem 43: 243-277.

236. Wan Dijk PWM (1979) Negatiwely clarged phospholipids and their position in the cholesterol affinity sequence. Biochim Biophys Acta $555: 89-101$.

237. Van der Vusse GJ, Roemen THM. Prinzen FW. Coumans WA, Reneman RS (1982) Uptake and tissue conient of fatty acids in dog myocardium under normoxic and ischemic conditions. Circ Res 50: $538-546$.

238. Van der Vusse GJ, Coumans WA, Van der Veen FH, Drake A, Flameng W, Suy R (1984) ATP. creatude phosphate and glycogen content in human myocardial biopsies: markers for the efficacy of cardioprotection during aorta-coronary bypass surgery. Wase Surg 18: 127-134.

239. Van der Vusse GJ, Reneman RS (1985) Pharmacological intervention in acute myocardial ischemia and reperfusion. Trends Pharmacol Sci 6: 76-79.

240. Van der Vusse GJ, Prinzen FW, Van Bilsen M, Engels W, Reneman RS (1987a) Accumulation of lipids and lipidmintermediates in the heart during ischaemia. Basic Res Cardicl 82 (Suppl. 1): 157-167.

241. Van der Vusse GI. Prinzen FW, Reneman RS (1987b) Disturbances in myocardial lipid homeostasis during ischemia and reperfusion. In: Activation, netabolisn and perfusion of the heart. Eds. Siclemann S, Beyar R. Martinus Nijloff Publishers. Dordrecht. pp: 665-681.

242. Van cler Vusse GJ, Van Bilsen M. Reneman RS (1989a) Is phospholipid degradation a critical exent in ischemia and reperfusion induced damage? News Physiol Sci (NIPS) 4: 49-53.

243. Van der Vusse GJ, Van Bilsen M, Sonderkanp T, Renenan RS (1989b) Hydrolysis of phospholipids and cellula integrity. In: Pathophysiology of severe ischemic injury. Ed. Piper HM. Marlinus Nijhoff Pulylishers, Dordrecht (in press).

244. Vasdew So, Biro GP, Narbaitz R, Kako KJ (1980) Membrane changes induced by early myocandial ischemia in the dog. Can J Biochem 58: 1112-1119.

245. Vermuri $R_{\text {n }}$ Philipson KD (1987) Phospholipid composition modulates the Na-Ca exchange activ" ity of cardiac sarcolemma in reconstifuted wesicles. Biochim Biophys Acta 937: 258-268.

246. Vermuri R, Mersel M. Heller M. Pinson A (1988) Studies on oxygen and volume restriction in 
cured cardiac cell - Possible rearrangemen of sarcolemmal lipid noheties during anoxia and ischemiawike states. Mol Cell Biochen 79: 39-46.

247. Verkleij AJ, Zwaal REA. Roelofsen B, Confurius P. Kastelin D, Van Deenen LLM (1973) The asymmetric distribution of phospholipids in the human ted cell nembrane. A combined study using phospholipases and freeze-etch electron microscopy. Biochin Biophys Acta 323: 178 . 193.

248. Verkleij AI. Ververgaert PHJTh (1975) The architecture of biological and artificial membranes as visualized by freeze fracturing. Ann Rev Phys Chem 26: 101-122.

249. Verkleij AJ, Humbel B. Siuder D. Muller M (1985) "Lipidic particle" systems as visualized by thin-section electron microscopy. Biochim Biophys Acta 812: $591-594$.

250. Verkleij AI, Post JA (1987) Physico-chemical properties and organization of lipids in membranes: their possible role in myocardial injury. Basic Res Cardiol 82 (Suppl 1): $85-91$.

251. Wacker I, Reiss H-D. Schnepf E. Traxel K, Bauer R (1986) Polar distribution of calcium- and phosphorus-rich globules induced by glutaralkdelyde/tanic acid fixation in the caulonema tip cell of the moss, Funaria hygrometrica: light microscopy, transmission electron microscopy (TEM), proton microprobe (PIXE), and electron spectroscopic imaging (ESI). Eur I Cell Biol 40: 94-99.

252. Wagner RC (1976) The effect of tannic acid on ellectron images of capillary endothelial cell unembrames. J Ultrastruct Res 57: 132-139.

253. Weglicki WB, Dickens BF, Mak IT (1984) Enhanced lysosomal phospholipid degradation and lysophospholipid production due to free radicals. Biochem Biophys Res Comm $124: 229-235$.

254. Weglicki WB. Low MG (1987) Phospholipases of the nyocardium. Basic Res Cardiol 82 (Suppl. 1): $: 107-112$.

255. Weibull C, Villiger W, Carlemalm $\mathrm{E}$ (1984) Extraction of lipids during freeze-substitution of Acholeplasma laidlawii-cells for electron microscopy. J Microsc (Oxford) 134: 213-216.

256. Wetzel MG, Scow RO (1984) Lipolysis and fatty acid transpon in rat heart electron microscopic study. Am J Physiol 246: C467.C485.

257. White $\mathrm{DL}$. Mazukiewicz JE. Barmett $\mathrm{RJ}$ (1979) A chentical mechanism for tissue staining by osmium tetroxide-ferrocyanicle mixtures. $J$ Histochem Cylochem 27: 1048-1091.

258. Whitmer IT, Idell-Wenger JA, Rovetto MJ. Neely JR (11978) Control of fatly acid metabolism in ischemic and hypoxic hearts. J Biol Chem 253:4305 4309.

259. Willerson JT, Scales F. Mukherjee A. Plat M. Templeton GH, Fink GS, Buja LM (1977) Abnormal nnyocardial fluid retention as an eatly manifestation of ischemic injury. Am I Pathol 87: $159-188$.

260. Wolf RA, Gross RW (1985) Identification of neutral active phospholipase $C$ which bydrolyses choline glycerolipids and plasmalogen selective phosplohipase $A_{2}$ in canine myocardium. I Biol Chem 260: 7295-7303. 
261. Wood 16 (1973) The effects of glutaraldehyde and osmium on the proteins and lipids of myetin and miochondria. Biochim Biophys Acta 329: 119-127.

262. Wrigglesworth JM (1985) Lipid dependence of membirane enzymes. In: Structure and properies of cell membranes: Ed. Penga Gh, CRC Press. Florida. pp: 137-157.

263. Wynants $J$, Van Belte $H(1985)$ Single-run high performance liquid chromatography of nucleo vides. mucleosides and major purine bases and its application to different tissue extracts. Anal Biocliem 144: 25,8-266.

264. Yanagishita T. Konno N, Geshi E. Katagiri $T$ (1987) Alterations in phospholipids in acute ischemic myocardium. Jap Circ J 51 : 41.50 .

265. Yeagle P1 (1989) Lipid regulation of cell membrane structure and function. FASEB J 3: 1833 . 1842 .

266. Zwaal RFA, Roelofsen B. Colley CM (1973) Localization of red blood cell membrane constituenis. Biochim Biophys Acta 300: 159-182.

267. Zwal RFA, Hemker HC (1982) Blood cell membranes and haemostasis. Haenostasis 11: 12-39. 


\section{SUMMARY}

It is well accepted that during myocardial ischemia and reperfusion membrane injury, as may be induced by metabolic alterations, plays an important role in the pathogenesis of irreversible cell damage. How and which, metabolic alterations attribute to the development of membrane injury is still subject of investigation.

In our studies we have focussed on membrane alterations during the early phases of ischemia and reperfusion, with emphasis on the development of multilamellar extrusions. Tannic acid has been advocated for the preservation of these lipidic extrusions from myocardial membranes. Since tannic acid has a complex and variable composition and little is known about the precise reaction(s) of tannic acid with phosphollipids, several methodologic questions had to be answered in relation to the possible effects of tannic acid based fixation during tissue preparation.

The findings of the various studies. described in this thesis, provide more insight into the reaction(s) of tannic acid with phospholipids and the impontance of tannic acid based fixation as a tool to asses the degree of myocardial ischemic (membrane) injury.

Tannic acid was found not only to reduce the extraction of phospholipid from unilamellar vesicles, but also to instantly induce aggregation when added to unilamellar vesicles (Chapter 2). This eventually leads to the formation of multilamellar vesicles, which were retrieved in thin sections. It appears that the interaction of tannic acid (in an approximately one-to-one molar ratio) with phospholipids, specifically containing a choline head group, may affect the electrostatic or hydration repulsive forces between the lipid bilayers and, hence, facilitating aggregation and/or fusion (Chapter 2 and 3 ).

A striking difference was observed between the interaction of tannic acid and unilamellar vesicles prepared from phospholipids extracted from rabbit or rat heans. This species difference was believed to be caused by differences in the content of phospholipids with a choline headgroup (including sphingomyelin, phosphatidylcholine and the plasmalogen phosphaticlalcholine), being substantially lower in rat hearts (Chapter 3). 
In ischemic and reperfused rabbit hearts multilamellar vesicles were found in both endothelial cells as well: as in myocytes. In myocytes $25-30 \%$ of the observed multilamellar vesicles were found to be extrucled from the sarcolemma, $25-30 \%$ from mitochondrial membranes and 40-50\% were located adjacent to or in lipid droplets (Chapter 4 and 5 ).

The total number of multilamellar vesicles observed in myocytes, and those extruded from mitochondrial membranes particularly, was found to be significantly increased in hearts rendered ischemic for at least 60 minutes. Multilamellar vesicles extruded from the sarcolemma were only found to increase upon reperfusion after the same period of ischemia. The number of multilamellar vesicles extruded from lipid droplets was found to be independent of the experimental condition (Chapter 4 and 5).

Since the number of multilamellar vesicles observed in the ischemic myocardium is positively related to the ischemic injury score, as based on several morphological parameters on a semi-quantitative scale, it is likely that these extrusions may be used to assess the clegree of ischemic (membrane) injury in a more quantitative way (Chapter 4).

It was remarkable that multilamellar vesicles could also be observed, though in small quantities, after periods of ischemia as short as 30 minutes or even in normoxically perfused control hearts. This indicates that multillamellar extrusions are not solely caused by the ischemic insult (Chapter 4 and 5 ).

All multilamellar vesicles observed were similar in morphological architecture (size-distribution and periodicity of membrane lamellae of the extrusions) irrespective of their site of location and the experimental condlition, indicating that there way of formation is based on similar mechanisms. The difference in size and number of multilamellar vesicles observed between rat and rabbit hearts is probably due to the different phospholipid content of the membranes in these species (Chapter 4,5 and 6 ).

Ii was demonstrated that in both ischemic and reperfused rat and rabbit hearts the significant increase in multilamellar extrusions correlates in time with 1) a significant depletion of ATP, 2) a signilicant accumulation of fatty acids (in particular arachidonic acid, an indicator of phospholipid. degradation) and 3) a significantly increased leakage of $\mathrm{LDH}$ (as estimated in rabbits). No significant alterations were observed in the tissue content of triacylglycerols and phospholipids (Chapter 5 and 6). 
Their might be a causal relation between the accumulation of amphipathic lipids during ischemia and the destabilization of myocardial membranes, as reflected by the increased number of multilamellar vesicles. These changes are likely to have functional consequences, as reflected by the leakage of LDH during reperfusion. Since the amount of arachidonic acid, an important constituent of phospholipids, accumulated during ischemia (nmolar range) is only indicating the degradation of a small a fraction of the total phospholipid content ( $\mu$ molar range), the findings in the present study provide evidence that only a very limited degradation of membrane phospholipids may cause significant membrane leakage (Chapter 5 and 6 ).

Further investigation of possible causal relations between the loss of membrane integrity, in which the glycocalyx and the cytoskeleton also plays a role, and biochernical and physico-chemical alterations during ischemia and/or reperfusion, could shed more light on the mechanisms involved in the development of myocardial cell injury. 


\section{SAMENVATTING}

Het is algemeen geaccepteerd dat membraan beschadiging tijdens myocardiale ischemie en reperfusie, welke bijwoorbeeld geinduceerd kan worden door metabole veranderingen, een belangrijke rol speel in de pathogenese van irreversibele cel beschadiging. Welke metabole veranderingen bijdragen tot de ontwikkeling van membraan beschadiging en hoe deze plaatswinden is onduidelijk.

Wij hebben in de hier beschreven studies onze aandacht gericht op membraan veranderingen lijdens de vroege fase van ischemie en reperfusie, met name op de ontwikkeling wan nullilamellaire membraan afsnoeringen. Tanninezuur wordt geacht de preservatie van deze lipid structuren in myocardiaal weefsel te bevorderen. Tanninezuur heeft een complexe en variabele samenstelling. Omdat er weinig bekend is over de exacte interactie tussen tanninezuur en fosfolipiden, werden er eerst enkele methodologische vragen beantwoord met betrekking tot de mogelijke effecten van tanninezuur tijdens weefsel-fixatie.

De bewindingen zoals beschreven in dit proefschrift, bieden meer inzicht in de effecten van tanninezuur op fosfolipiden en de betekenis van tanninezuur bevattende fixaties als middel om de mate van myocardiale (membraan) beschadiging ten gevolge van ischemie te kwantificeren.

Tanninezuur reduceert niet alleen de extractie van fosfolipiden uit unilamellaire lichaampjes, maar induceert tevens aggregatie, fusie en de formatie van multilamellaire structuren. welke in dunne coupes terug te vinden zijn (Hoofdstuk 2). Het is warschijnlijk dat de interactic van tanninezum met fosfolipiden (in ongeveer een $1: 1$ molaire ratiol, in het bijzonder die fosfolipiden welke een choline hoofdgroep bevatten. de electrostatische en/of hydratatie afstotingskrachten tussen de lipid bilagen beinvloedt en zo aggregatie en/of fusie bevorden (Hoofdstuk 2 en 3).

Unilamellaire lichaampjes vertoonden een opvallend verschil voor wat betreft de interactie met tanninezuur, ahankelijk van het feit of de fosfolipiden waarvan ze gemaakt werden, geëxtraheerd waren wit konijneharten of uit ratteharten. Dit verschil wordt waarschijnlijk veroorzaakt door de hoeveetheid fosfolipiden met een choline hoofdgroep (zoals sphingonyeline, phosphatidylcholine en de plasmalogen phosphatidalcholine), welke substantieel lager was in ratteharten (Hoofdstuk 3). 
In ischemische en gereperfundeerde konijneharten werden multilamellaire structuren aangetroffen in endotheel cellen alsook in de cardiomyocyten. In dit laatste celtype was de relatieve verdeling van multilamellaire lichamen als volgt: $25-30 \%$ van de structuren was afkomstig als uitstulping van de plasmalemma, 25-30\% van mitochondrale membranen en $40-50 \%$ werd gevonden in of nabij intracellulaire vetdruppels (Hoofdstuk 4 en 5).

Het totaal aantal geobserveerde multilamellaire membraan uitstulpingen in myocyten en in het bijzonder die welke afgesnoerd waren van mitochondriale membramen nam significant toe nadat harten ischemisch waren gemaakt voor een periode van tenminste 60 minuten. In tegenstelling tot de multilamellaire lichampjes welke afgesnoerd waren van mitochondriale membranen, nam het antal membraan afsnoeringen van het sarcolemma niet significant toe onder deze ischemische condities, Werden echter de harten gereperfundeerd na een ischemische periode van tenminste 60 minuten, dan werd er wel een significante toename waargenomen. Het aantal multilamellaire structuren in of nabij vetdruppels bleek onafhankelijk te zijn van de (patho)fysiologische conditie van het hartspierweefsel (Hoofdstuk 4 en 5).

Het is waarschijnlijk dat multilamellaire membraan afsnoeringen gebruikt kunnen worden voor de bepaling wan de mate van (membraan) beschadiging ten gevolge van ischemie. Het aantal multilamellaire lichaampjes in het ischemische hartspierweetsel is namelijk positief gerelateerd aan de ischemische schade-score, welke gebaseerd is op diverse morfologische kenmerken (Hoofdstuk 4).

Alle multilamellaire structuren hadden een vergelijkbare morfologische architectuar (grootte-distributie en periodiciteit van de lamellen) onafhankelijk wan de subcellulaire localisatie en de experimentele conditie. Dit suggereert dat de manier van multilamellaire lichaamvoming gebaseerd is op een overeenkomstig mechanisme. Hel verschil tussen ratte en konijneharten voon wat betreft de grootte en aantal multilamellaire structuren wordt waarschijnlijk veroorzaakt door een verschil in de fosfolipid samenstelling van de membranen in deze diersoorten (Hoofdstuk 4 en 5 ).

Aangetoond werd dat in zowel ischemische als in gereperfundeerde ratte- en konijneharten de significante toename van het aantal multilamellaire lichampjes gerelateerd is in tijd met 1) een significante depletie van ATP, 2) een significante accumulatie van vetzuren (arachidonzuur, een indicator woor fosfolipid afbraak, in het 
bijzonder) en 3) een significante toename van de LDH lekkage (alleen in konijnen bekeken). Er werden geen significante veranderingen waaregenomen in de hoeveelheden triacylglycerols en fosfolipiden welke in het weefsel aanwezig zijn (Hoofdstuk 5 en 6).

Het is mogelijk dat er een causaal verband bestaat tussen de accumulatie van amphipathische lipiden en de destabilizatie van myocardiale membranen tijclens ischemie, zoals weerspiegeld in de toename van multilamellaire afsnoeringen. Deze veranderingen hebben watschijnlijk ook functionele consequenties, zoals blijkt uit de lekkage van $L D H$ tijdens reperfusie. Omdat de significante stijging van de hoeveelheid arachidonzuur (nmol range) slechts de afbraak wan een kleine fractie van cle totale fosfolipiden inhoud (umol range) weergeeft, suggereren de behaalde resultaten dat een beperkte afbraak van fosfolipiden reeds tot een significante membraan lekkage aanleiding kan geven (Hoofdstuk 5 en 6).

Verder onderzoek naar de mogelijk causale relatie tussen het verlies van membraan integriteit enerzijds, waarbij de glycocalyx en het membraan-cytoskeletaire netwerk ook een roll spelen, en de biochemische en physico-chemische veranderingem tijdens ischemie en/of reperfusie anderzijds, kan meer licht werpen op de mechanismen betrokken bij de ontwikkeling van myocardiale cel beschadiging. 


\section{CURRICULUM VITAE}

Ad Schrijvers werd op 10 juli 1957 te Aalst geboren. In 1974 behaalde hij het MAVO diploma aan de Hoge Vught MAVO te Breda. Na het HAVO diploma (1976) behaalde hij in 1978 het VWO diploma aan de Newman-IJpelaar Scholengemeenschap te Breda. In 1978 begon hij zijn studie Biologie aan de Rijksuniversiteit te Leiden. In 1981 slaagde hij yoor het kandidaatsexamen Biologie, waarna een aanvang gemaakt werd met het hoofdvak Immunohaematologie onder leiding van Dr. J.W. Bruning (AZL) en de bijwakken Pathofysiologie en Celbiologie onder leiding van, respectievelijk Dr. J.J. Emeis (Gaubius) en Dr. W. de Priester (RUL). In 1984 slaagde hij voor het doctoralexamen Biologie, waarna hij werkzaam was op de afdeling Fysiologie (i.s.m. Pathologie/EM) van de Rijksuniversiteit Limburg te Maastricht. Sinds Augustus 1989 is hij werkzaam op de afdeling Keel-, Neus- en Oorheelkunde, sectie tumor biologie, van de Vrije Universiteit te Amsterdarn. 


\section{BIBLIOGRAPHY}

1. A.H.G.I. Schrivers, L.H.E.H. Snoeck. M.C.A. Stuart, P.M. Frederik and R.S. Reneman (1988). Gutaraldehyde-lysine fixation for studying cardiac membranes. Ultranicroscopy. 24: p 447 (Abstr。)

2. A.H.G.J. Schrifvers, V.V.Th. Heijnen, P.M. Frederik and R.S. Reneman (1988). Immunocytow chemical localization of $\left(\mathrm{Na}_{1} \mathrm{~K}\right)$ ATP ase in cardiac lissue. Ultramicroscopy. $24: \mathrm{p}$ 447 (Abstr.).

3. A.H.G.J. Schrijuers, P.M. Frederik, V.V.Th. Heijnen and R.S. Reneman (1988). Increased number of multilamellar extrusions after prolonged ischemia and reperfusion in rabbit myocardium. J, Mol. Cell. Cardiol., 20 (Suppl.V): S.31 (Abstr.).

4. A.H.G.J. Schrijvers, P.M. Frederik, M.C.A. Stuart and R.S. Rememan (1988). The reaction of lannic acid with phospholipids - A fixation or a nodification of the ultrastructure. J. Moll. Cell. Cardiol., 20 (Suppl.V): S.76 (Abstr.).

5. A.H.G.J. Schrijuers, L.H.E.H. Snoeckx, M.C.A. Stuart. P.M. Frederik and R.S. Reneman (1988). Examination of glutaraldehyde-lysine fixed ischenic myocardiun. J. Mol. Cell. Cardiol., 20 (Suppl.V): S.31 (Abstr.).

6. A.H.G.J. Schrijvers, P.M. Frederik, M.C.A. Stuart. G.J. van der Vusse and R.S. Reneman (1989). Dual effect of tannic acid on the preservation and ultrastructure of pliosphatidyl choline vesicles. Mol. Cell. Biachem. 88: 91-96.

7. P.M. Frederik, M.C.A. Stuart, A.H.G.J. Schrijwers and P.H.H. Bomans (1989). Thin film formation and the imaging of phospholipid by cryo-electron microscopy. In: The science of biological specimen preparation, SEM, A.M.F. O'Hare (ed.) (in press).

8. A.H.G.J. Schrijvers, P.M. Frederik. M.J.M. de Groot, Y.V.Th. Heijnen, G.J. van der Vusse and R.S. Reneman (1989). Induction of multilamellar wesicles in the isolated rabbit heart after ischemia and reperfusion. Ultranicroscopy 27: 216-217 (Abstr.).

9. G.J. van der Vusse, M.J.M. de Groot, P.H.M. Willemsen, M. van Bilsen, A.H.G.J. Schrijuers and R.S. Reneminan (1989). Degradation of phospholipids and triacylglycerol, and accumulation of farty acids in anoxic myocardial tissue, distupted by freeze-thawing. Mol. Cell, Bochen. 88: 83.90.

10. A.H.G.J. Schrijuers, P.M. Frederik. M.C.A. Suart, K.N.J. Burger, V.V.Th. Heijnen, G.J. van der Vusse and R.S. Reneman (1989). The formation of multilamellar vesicles by the addition of tamnic acid to phosphatidylcholine containing small unilamellar vesicles. J. Histochem. Cylociliem. (in press).

11. A.H.G.J. Schrifwers, M.J.M. de Groot, V.V.Th. Heijnen, G.J. van der Vusse, P.M. Frederik and R.S. Reneman (1989). Ischemia and reperfusion induced multilamellar vesicles in isolated rabbit hearts: Time correlation between morphometric data and metabolic alterations. J. Mol. Cell. Cardiol. (Provisionally accepted). 


\section{DANKWOORD}

Het in dit proefschrift beschreven onderzoek werd uitgevoerd, in het kader van het myocard-projekt, bij de Rijksuniversiteit Limburg te Maastricht. Graag maak ik van de gelegenheid gebruik iedereen te bedanken, die heef bijgedragen aan het tot stand komen van dit proefschrift. In het bijzonder wil ik bedanken:

Mijn promotores Rob Reneman en Ger van der Vusse, voor jullie begeleiding en stimulerende gesprekken met name met betrekking tot het schrijven van de artikelen en dit proefschrift. Mijn co-promotor Peter Frederik voor je bijzonder plezierige bege-leiding, je waardevolle adviezen en leerzame discussies en de niet-aflatende stroom van ideeën. Jouw steun is van essentieel belang geweest voor de totstandkoming van dit proefschrift. De referenten wil ik bedanken voor het kritisch beoordelen van het manuscript. Viviane Heijnen. Marc Stuart, Paul Bomans en Wim Busing voor hun praktische ondersteuning en fijne samenwerking. Monique de Groot en Kurt Burger voor hun praktische bijdrage. Theo Roemen. Peter Willemsen en Wil Coumans woor het uitvoeren van de biochemische analyses. Frank Peeze-Binkhorst woor de vele stimulerende discussies en belangstelling. Judith Stouten, Jos Beliën en Henk van Rie voor hun persoonlijke interesse. De medewerkers van de vakgroepen Fysiologie en Pathologie voor de goede samenwerking en de prettige werksfeer. Tot slot wil ik mijn familie en vrienden bedanken voor hun belangstelling en de steun die zij voor mij zijn geweest. Met name mijn ouders (en schoonouders) wil ik bedanken voor het feit dat zij mij altijd gestimuleerd hebben in mijn studie en onderzoek. Vooral mijn wrouw Anita en mijn zoontje Sander bedank ik voor hun geduld en begrip voor het feit dat ik ze de afgelopen periode ongetwijfeld te weinig aandacht heb geschonken. 